\title{
CRITICAL INSIGHTS INTO GENDER MAINSTREAMING IN NON-GOVERNMENTAL ORGANIZATIONS BASED IN VIETNAM
}

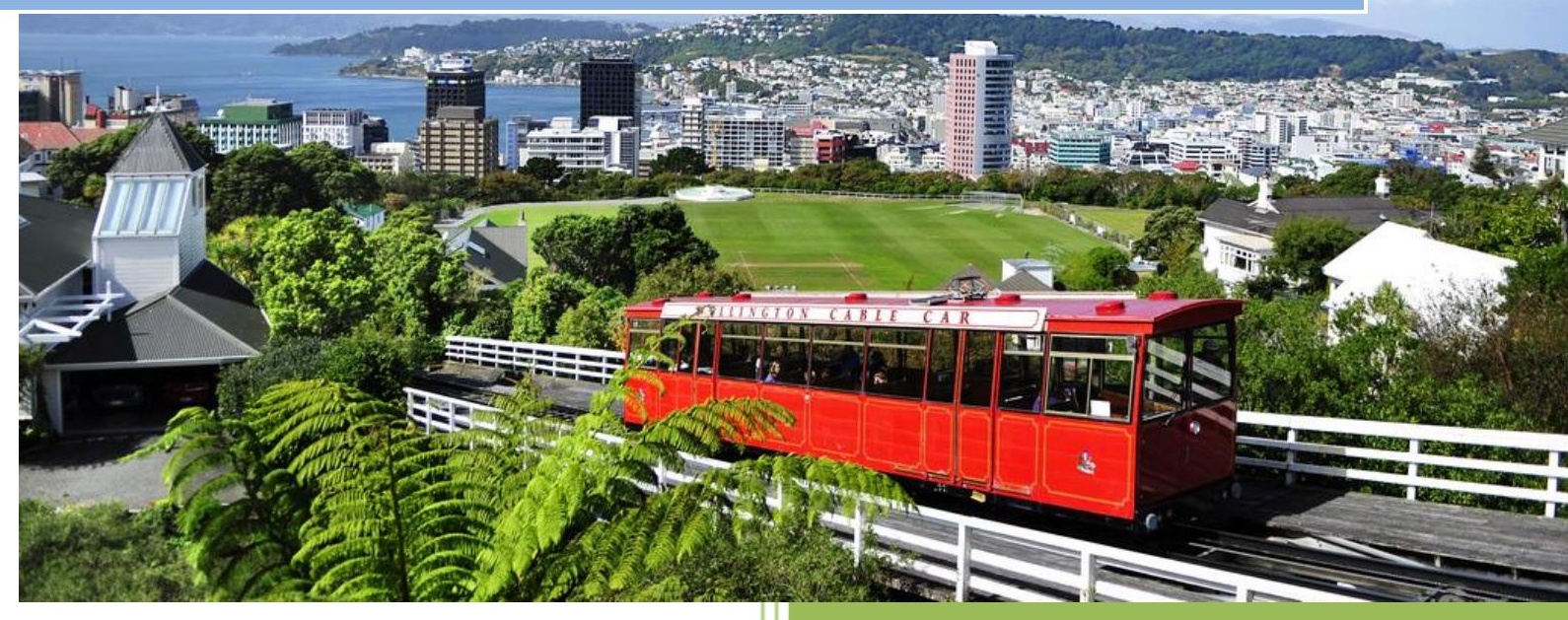

\section{Binh Thi Le}

A thesis submitted to Victoria University of Wellington in partial fulfilment of requirements for the degree of Masters in Development Studies 



\section{TABLE OF CONTENTS}

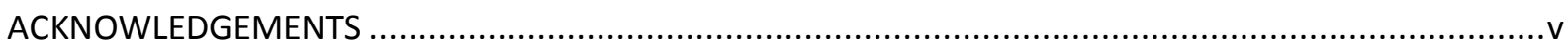

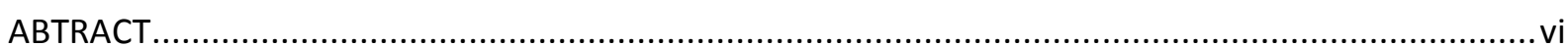

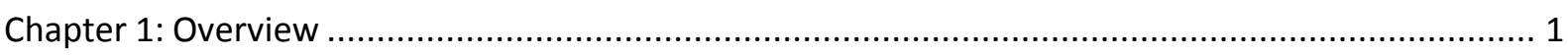

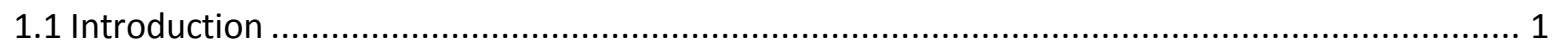

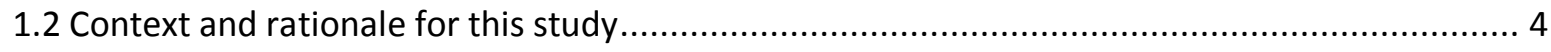

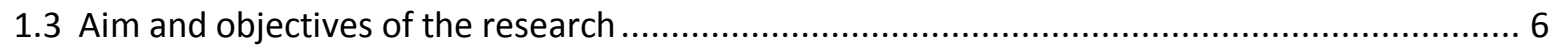

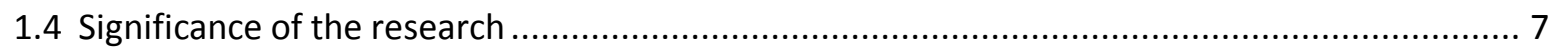

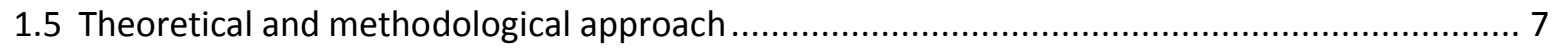

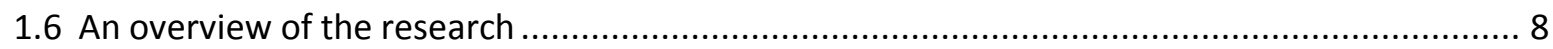

Chapter $2:$ Theoretical framework and literature review ...................................................10

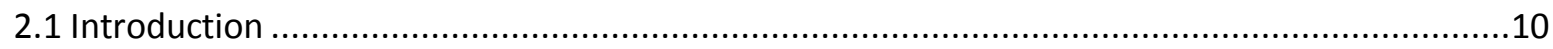

2.2Review of different approaches or feminist theories to gender equality .............................10

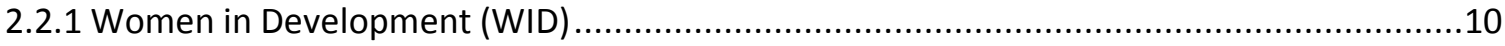

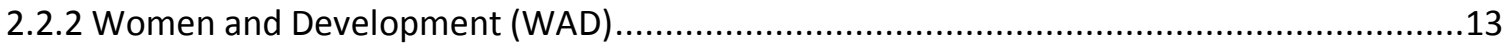

2.2.3 Gender and Development (GAD) ............................................................13

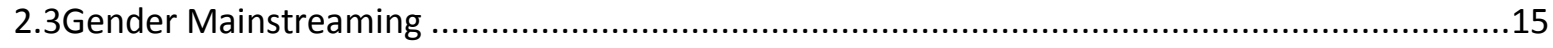

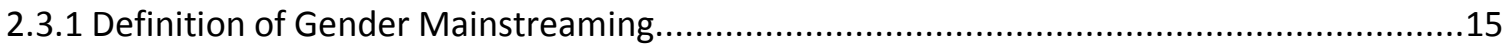

2.3.2 Critical insights into waysof gender mainstreaming ..............................................16

2.4International institutional frameworks on gender equality ..........................................18

2.5Non-governmental organizations and gender work ...................................................19

2.5.1 The history of NGOs and the rise of their involvement in development work ..................19

2.5.2 Gender mainstreaming in global NGOs ......................................................22

2.6 Gender Mainstreaming in practice: Success or Failure? .............................................23

2.6.1 Lack of a conceptual clarity around the key terms...........................................24

2.6.2The lack of political will for institutional change....................................................26

2.6.3 Insufficient attention to and/ or the resistance to gender mainstreaming among

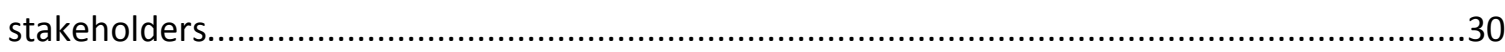

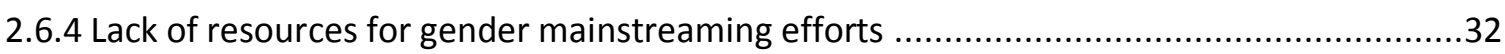

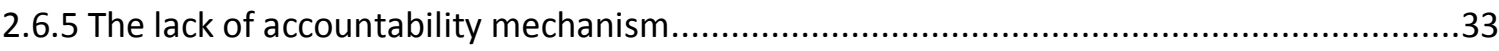

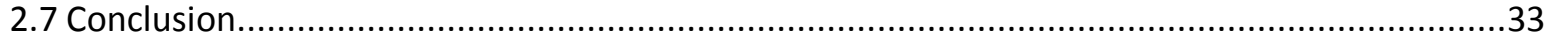

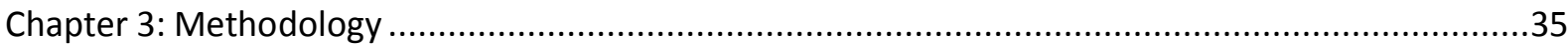


3.1 Introduction

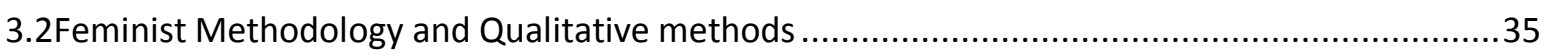

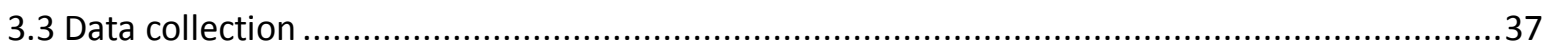

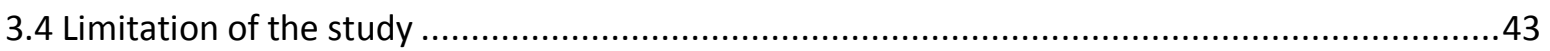

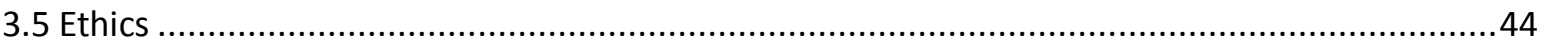

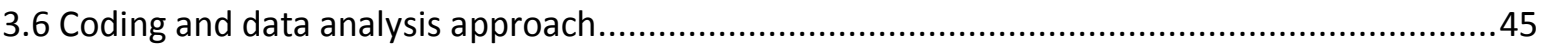

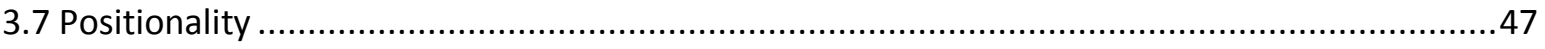

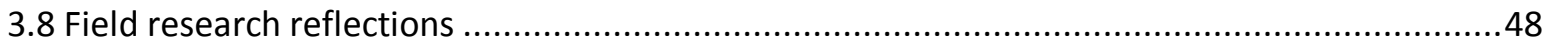

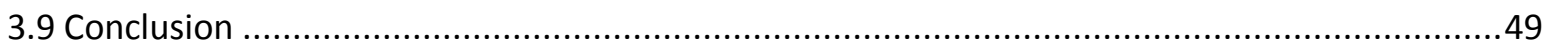

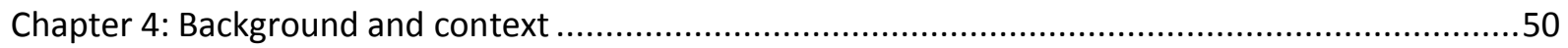

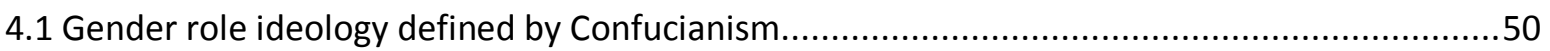

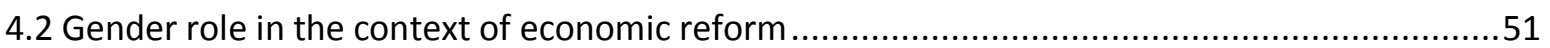

4.3 Institutional Frameworks for the Advancement of Women .....................................................54

4.4 Progress vs Impediment in addressing Gender Issues in Vietnam ..........................................60

4.5 Development intervention for gender equality in Vietnam ................................................67

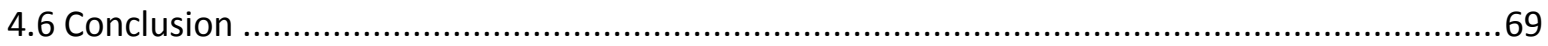

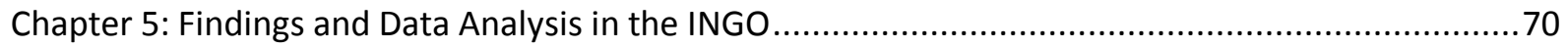

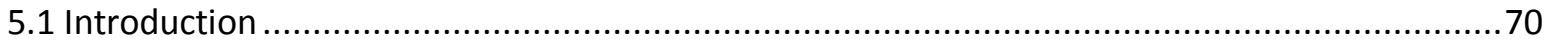

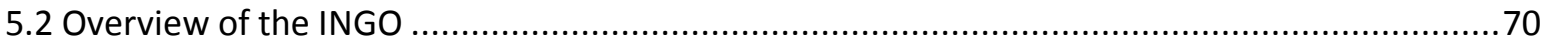

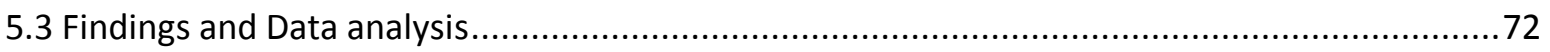

5.3.1 Locating entry points for Gender Mainstreaming (GM) process .......................................72

5.3.2 Institutional challenges caused by a superficial and inconsistent process ...........................77

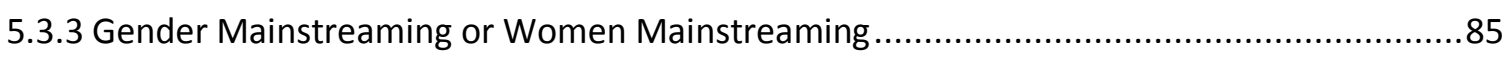

5.3.4 Opportunities for INGO's greater contribution to gender equality in the long-term ..........87

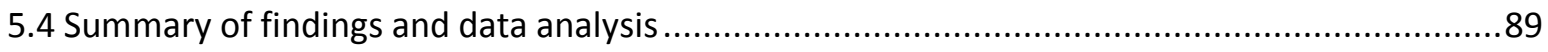

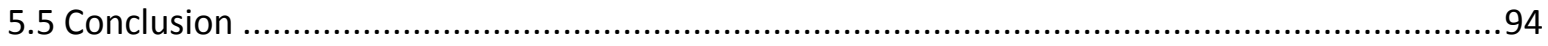

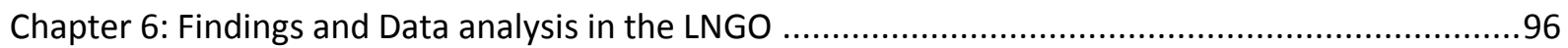

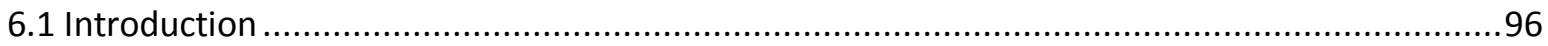

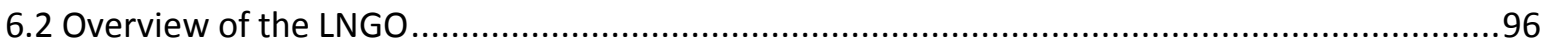

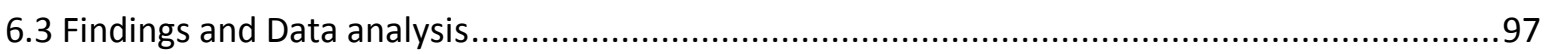

6.3.1 Institutionalizing gender in the organizational policies...................................................97

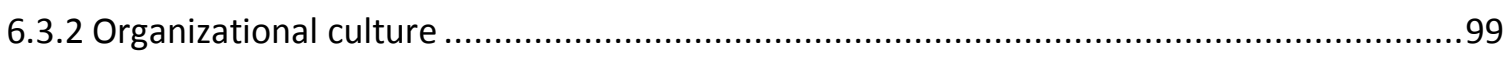

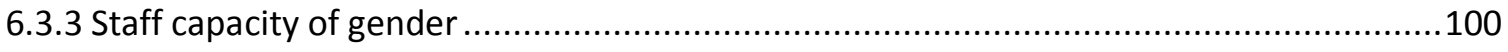

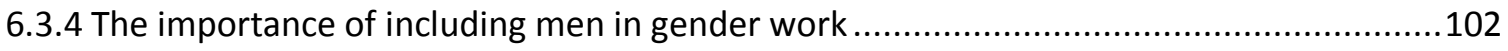




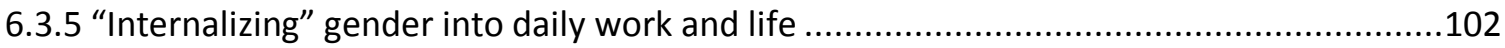

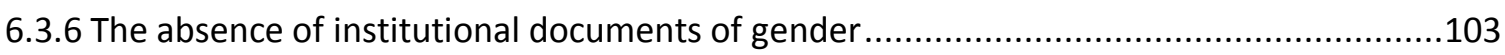

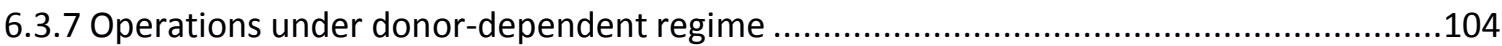

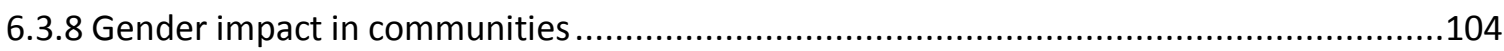

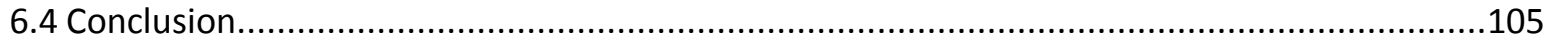

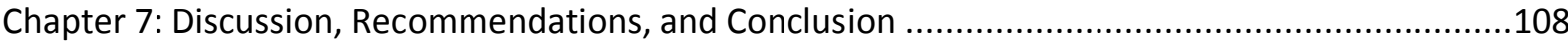

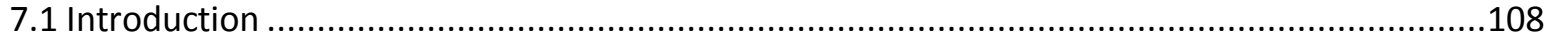

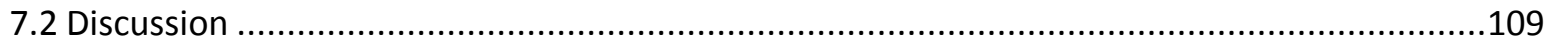

7.2.1 Where are NGOs in the process of gender mainstreaming? .........................................109

7.2.2The unclear boundary between WID and GAD approach .........................................112

7.2.3Taking men's issues and men's involvement on board to address gender inequality ........113

7.2.4 NGOs: An organizational culture makes positive gender impacts in wider society?! ........116

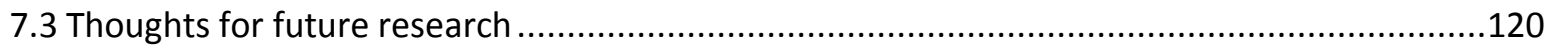

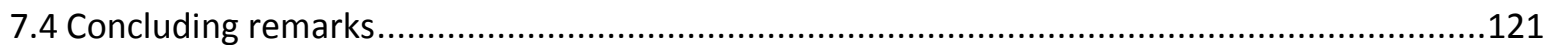

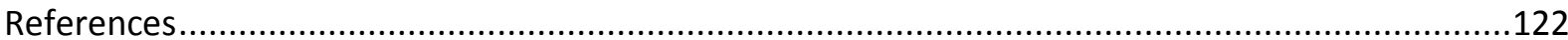

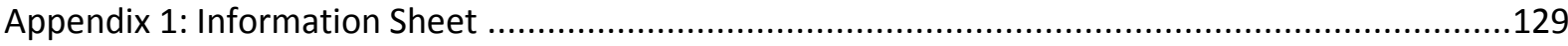

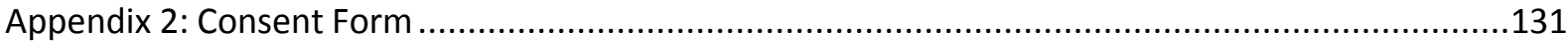

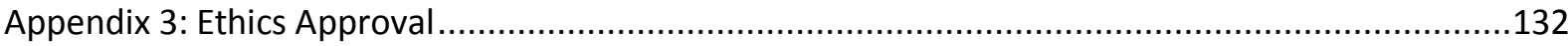




\section{List of Figures}

Figure 1.1. Thesis outline $\quad 9$

Figure 2.1. Dynamics between top-down and bottom-up forces of change 17

$\begin{array}{ll}\text { Figure 2.2. DFID gender mainstreaming strategy } & 18\end{array}$

Figure 2.3. Gender at work: what are [organizations] trying to change? 28

Figure 5.1. Supervision lines of the GTF in the INGO 75

Figure 5.2. Technical preparedness of the INGO to take off the gender 90

mainstreaming process

Figure 5.3. Power relations in decision-making process 92

Figure 5.4. The progress to gender equality and factors influencing that progress 92

Figure 6.1. Illustration of the 'multifunctional' staff model in the LNGO 101

Figure 6.2. Summary of strategies used to achieve double-tier goals in the LNGO 106

Figure 7.1. The iceberg of organizational structure 116

Figure 7.2. The organizational likelihood of promoting gender equality 117

\section{List of Tables}

Table 3.1. List of Informants $\quad 40$

Table 4.1. Level of Schooling Completed by Age and Gender (percentage 62 completed)

Table 4.2. The proportion of men and women aged over 15 holding a job in selected employment areas

Table 4.3. Proportion of females employed against total labour force of 2007

\section{List of boxes}

Box 4.1: Vietnam Development Goals (SEDP 2006 to 2010) 55 


\section{ACKNOWLEDGEMENTS}

This thesis would not have been possible without the endless support of my supervisor, Dr. Sara Kindon. First of all, I would like to extend my profound gratitude to her for providing me with encouragement, thoughtful feedback, and invaluable guidance throughout the research. Her patience and closeness always made me feel easy about this 'supposed-to-be tough' journey. Her encouragement and wisdom contributed significantly to the outcome of this research.

I am also grateful to my sponsor, the New Zealand Agency for International Development (NZAID), for providing me with full support to be in Wellington for more than two years so that I could undertake the Masters degree in Development Studies at Victoria University of Wellington and conduct my field research in Vietnam.

Also, I would like to express my appreciation towards the Program's Director - John Overton, and its Lecturer - Andrew McGregor who made me more interested in studying Development Studies and advised me when needed.

I wish to thank my 'DevStudies Cats' for making me enjoy being in a friendly and supportive setting and their classmate, especially Adele, Kristine and Karly for their constructive discussions and opinions on my thesis.

I would like to highly appreciate all interviewees who took their time with me and shared practical knowledge, ideas and experiences.

Of course, for making the whole journey more courageous, thank you to my close Vietnamese friends at home and here in Wellington, who always stood by me and motivated me. Their academic advice and support were very crucial for the completion of my research.

Last but not least, my great gratitude and love is extended to my parents and siblings who kept their good health and happiness at home, so that I could be released to concentrate on my study here. Without their spiritual support by distance, I would not be able to get through my two years of study time here. 


\begin{abstract}
ABTRACT
In Vietnam, Confucian doctrine has traditionally influenced gender issues. Although, the determination of Government has brought about considerable improvements in gender equality in society, there remains a need for interventions from a variety of stakeholders including Non-Governmental Organizations (NGOs). Recently, both International NGOs (INGOs) and Local NGOs (LNGOs) have increased in scope, size, and resources to address development issues in general and gender issues in particular in the specific context of Vietnam. They all play a pivotal role in delivering services and advocacy in an effort to enable Vietnamese people to live in a society of justice and well-being. However, there continues to be a gap between the rhetoric and practice of these organizations for both internal and external reasons.
\end{abstract}

This research analyses the process of integrating gender into an INGO and an LNGO's policies and development projects drawing on semi-structured interviews with staff in these organizations. The study explores the extent to which these NGOs use gender mainstreaming as a tool to achieve the goal of gender equality in their own organization and project sites. What challenges and opportunities for each organization exist?

The findings indicate that in reference to gender mainstreaming, these NGOs, more or less, have encountered a series of common obstacles found in literature from other parts of the world. They also highlight the importance of the approach to gender work taken by NGOs. Each approach must be based on the specific conditions and characteristics of each organization and more importantly adaptable to the context, culture, and traditions of Vietnam. As a part of gender mainstreaming, NGOs are starting to pay attention to the work/home divides of their staff as a critically important element in organizational culture aiming to get the organization in order first before integrating gender into other dimensions of their development work. Both NGOs have the strong determination to have more male staff in office and involve more men in projects to address gender issues. Furthermore, the lack of openness and dialogue on implementing gender mainstreaming in the NGO community makes a part of the findings. These particular aspects of organisational practice were highlights of this thesis. 


\section{Chapter 1: Overview}

\subsection{Introduction}

The research aims to investigate the extent to which NGOs based in Vietnam mainstream gender in their development work. Data were generated using a case study approach, which employed semi-structured interviews. Secondary data, from Vietnamese newspapers, articles, and documents from the Vietnamese government and the international development community were used to capture a wider understanding of both gender issues in Vietnamese society as well as efforts taken by various stakeholders to address them. A desk review, including organizational analysis of NGO project documents, gender strategies and other relevant government documents complemented primary and secondary data sources

Gender inequality has been seriously impeding the course of poverty reduction in many places around the world. A persistent issue, it has been put on working agendas in both international and local arenas participated by various actors such as politicians, development practitioners, academia, and others. It has also drawn attention from many researchers around the world. The serious concerns about gender issues have led to the development of relevant feminist theories and the issuance of international frameworks over time. These have laid the foundation for bringing gender consideration into the centre of development. In doing so, women are no longer expected to be marginalized, and men and women ideally share equal access, involvement, and benefits from the development process.

A series of international frameworks has taken gender into consideration. For example, the Millennium Development Goals (MDGs) agreed on by 180 countries in 2000 have eight goals, of which Goal \#3 is specific to gender equality and women's empowerment. Other instruments also seek to bring gender into the mainstream. These are the Declaration on the Elimination of All Forms of Discrimination against Women, in 1967, and the Convention on the Elimination of All Forms of Discrimination against Women (CEDAW), in 1979, adopted by the United Nations (UN). In 1995, the Beijing Platform for Action was signed by representatives of 189 governments, which, like CEDAW, identifies a list of areas of special concern to women. Gender has been taken into account to strengthen the effectiveness of 
aid delivery through the Paris Declaration and the Accra Agenda for Action (2008) which identified gender equality as a priority development issue.

These platforms for greater gender equality are driven by feminist theories which primarily consist of Women in Development (WID), Women and Development (WAD), and Gender and Development (GAD). In this thesis, I have reviewed these theories to reach an understanding of their core implications and applications in practice (see Chapter 2). The limitations of each approach have been examined while making comparisons between them. It occurs to me that WID and GAD are popularly used in the development mainstream to solve gender issues. To my knowledge, WID and GAD are interchangeable and supplementary to each other for the sake of addressing gender inequality. Still, women-specific interventions under WID are crucial if women are to be free of marginalization and subordination. GAD entered the mainstream as the most effective approach to addressing gender inequality for how it examined the social construction of gender relations while acknowledging men's contribution to solving inequity and social injustice. Moreover, the approach acknowledged both the internal and external roles of women. Going beyond that, it sought to transform unequal gender relations to ensure sustainable development for women and men through bolstering women's voices in politics.

GAD was simplified with the adoption of gender mainstreaming at the UN Conference on Women, held in Beijing in 1995. Gender mainstreaming is conceptualized as a strategy for institutionalizing and integrating gender concerns into the mainstream to provide men and women with equality in the legal, social, economic, and political rights based on their different needs and priorities. As a result of this process, the two sexes should have equal opportunities to fulfil their rights and participate in national, political, economic, social and cultural development(CIDA, 1999 cited in A. Moser \& Moser, 2005). Gender mainstreaming was widely adopted by governments, development organizations, civil societies, and NGOs because it was expected to be an ideal replacement for traditional tools used in addressing gender issues. 
As alternative agents for development, NGOs have become popular providers of humanitarian assistance and other services such as watchdog, education, and advocacy in the global aid system. NGOs are known for an ability to reach the marginalized and poor that governments cannot(Chege, 1999). NGOs have continued to swiftly increase in size, resources, and scope to address development issues exacerbated by macro policies such as structural adjustment and neo-liberalism in developing countries. It is evident that gender issues are one of their focuses. Subsequently, NGOs have adopted gender mainstreaming as their strategy for systematically integrating gender considerations into organizational policies, strategies, plans and projects. In addition, staff of NGOs have gender norms, attitudes and practices of their own (Manderson \& Mark, 1997; Tiessen, 2007; Wallace, 1998). NGOs are generally regarded as a better actor to address inequality and injustice at small scales than government.

A decade after the emergence of gender mainstreaming, a review was conducted to assess its progress in development institutions including NGOs. Unfortunately, the result revealed ambiguity in the effectiveness of its practice(Dawson, 2005; Desai, 2005; Jahan, 1995; Kabeer, 1994, 1999, 2005; A. Moser, 2007; A. Moser \& Moser, 2005; C. Moser, 2005; Tiessen, 2004, 2007; Wallace \& Wendoh, 2005). Such ambiguity was a result of four main factors:

1. Lack of a conceptual clarity around key terms

2. Insufficient attention to and/ or the resistance to gender mainstreaming among those concerned

3. Lack of resources dedicated to gender mainstreaming efforts

4. Absence of an accountability mechanism

Given that gender inequality remains an impediment to global development and that the launching of gender mainstreaming was expected to be a prescription for this issue, it is worth noting that these findings would provide references for NGOs in Vietnam considering adopting gender mainstreaming. Those working in and for gender equality in Vietnam, therefore, have an opportunity to optimize gender mainstreaming in the local context as detailed in the following section. 


\subsection{Context and rationale for this study}

In Vietnam, the culture and traditions are largely influenced by Confucian ideology, which determines the roles of women and men in family and public life. Within this ideology, women are tied to "four virtues" (Tứ Đức) - hard work, beauty, fidelity and passivity - and "three obediences"(Tam Tòng), to fathers, husbands and sons(Hoang, 2005). Specifically, women are responsible for serving their husbands, bearing and rearing children, doing housework, and looking after parents-in-law in old age. They are confined to their fathers, their husbands, and their oldest sons upon their husband's death. This patrilineal and patrilocal kinship system causes son-preference which has proven to be a very pervasive and hard-to-dilute issue, particularly in rural areas of Vietnam. Parents prefer to have sons because of their responsibilities to carry on family lines and names, perform ancestor worship, and take care of parents in old age. This household patriarchy still causes major cultural and social obstacles to efforts aimed at increasing the standing of women (ADB, 2002; Hoang, 2005; Knodel, Manh Loi, Jayakodil, \& Tuan Huy, 2004; Teerawichitchainan, Knodel, Manh Loi, \& Tuan Huy, 2008).

I am a young Vietnamese woman, born into a family of five children (including me). My parents used to live in a village before moving to the capital town of a Northern Province in Vietnam. My father was a fulltime official in the State sector while my mother worked on a farm. I grew up with the thought that 'a Goody Two-Shoes Path' of a woman involves domestic work, child-bearing and rearing, working in the farm (if in rural areas), and serving one's husband and his family. All of these responsibilities are put on one woman. My perception was derived from observing both what my mother did in my own family and what other women like her did around me. I think my family is an exemplary Vietnamese family in lots of ways. The most notable point here is that my father made the important decisions for the family while my mother never passed comment. My father and we children still don't know what my mother likes, as she never makes any requests for herself. She receives great compliments for being a good traditional Vietnamese woman. Being a wife and a mother, she has been paid with great gratitude and love from her husband and children. Importantly, she always felt happy with all the roles she has performed. However, 
when I matured, it seemed to me that these things were set out as a woman's destiny, and therefore my destiny, as if it would be no use commenting on or reasoning with them. Interestingly enough, the older I get, the more I ponder this scenario. I am curious to see if these rules that govern a woman's life are really unchangeable and non-transformable. I wonder, is there a life path outside of a kitchen and a house that a Vietnamese woman can choose to take? And if so how can gender roles be changed?

I have a great interest in reading the "agony columns" (muc tam su), posted daily on Vietnamese e-newspapers such as http://afamily.vn/, http://vnexpress.net/, http://dantri.com.vn/, and http://vietnamnet.vn/. They are all reputable e-newspapers in Vietnam, and each attracts a huge number of Vietnamese audiences at home and overseas. Recently, very controversial threads around women and men's issues, and their private and social lives in Vietnam, have been posted. From my observation, Vietnamese women appear to be more open than ever before, speaking about the highs and lows of their private lives publicly. Many of these women are beginning to fight against their personal traps, which were built up by gender stereotypes in Vietnamese cultural and traditional norms. In addition, some men also pay attention to these stories and share their opinions, attitudes and reactions and speak back to their difficulties with how their masculinity has been defined under this patriarchal system. Positively, this kind of forum can be seen as an encouraging sign that women and men wish to let go of the deep influence of Confucian doctrine on gender roles, and are taking a step towards raising people's awareness. The proverb "many a little makes a mickle" conveys the contribution of the mass media to the efforts towards improving gender equality in Vietnam.

From the governmental side, Vietnam has considered gender issues a top priority in its development agenda since 1930. It has adopted Gender and Development as an approach and gender mainstreaming as a strategy. Although mainstreaming gender issues in the health and education sectors has gained some remarkable achievements, major current concerns are potentially undermining the process of gender mainstreaming in Vietnam. The UN's 2009 report outlined the confusion of key concepts, such as mistaking 'gender' for 'women', and the lack of awareness about the importance of gender mainstreaming as the most effective and popular approach to gender equality(NCFAW, 2004b). For development 
institutions in Vietnam such as the UN system, the 2008 UNCT Vietnam Gender Audit noted some key challenges including the lack of awareness on and capacity to enable gender policy among its staff, lack of accountability at management level to gender equality outcomes, lack of management support, and insufficient investment in staff capacity to enable gender equality outcomes, among others. All these are challenges to the UN goal of achieving gender equity and equality in Vietnam(UN, 2009).

International non-government organizations (INGOs) and local non-government organizations (LNGOs) in Vietnam have made great efforts, together with the government, to address development issues including gender. In recent years, INGOs and LNGOs have been very actively involved in GAD in Vietnam. They usually implement small and short term projects, some of which are specific to women. This approach is better equipped to dealing with sensitive areas like gender-based violence(ADB, 2002). In addition, NGOs play a key role in addressing gender inequality(NCFAW, 2004b). However, it seems that there has not been any research into how they are approaching this work and how effective they are.

\subsection{Aim and objectives of the research}

This gap and the ambiguities around GAD and gender mainstreaming above inspired me to carry out this research with the specific aim, objectives and expected outcomes as follows:

\section{Thesis Aim}

This research aimed to examine the extent to which NGOs based in Vietnam mainstream gender in the development work, or, put as a direct question, is the theory translating into practice?

\section{Research Questions}

1. To what extent has gender been mainstreamed within the NGOs' policies, practices, and procedures?

2. To what extent does each organization mainstream gender concerns in its specific projects?

3. What opportunities exist for NGOs' gender work to contribute to addressing gender inequality in Vietnam? 
4. How is gender considered in the organizational culture of NGOs?

\section{Research objectives}

Answering those questions helped me to:

1. Elaborate on the institutionalization of gender mainstreaming in the participating development NGOs in Vietnam

2. Provide critical insights into the challenges and opportunities these organizations have in their efforts to mainstream gender

3. Explore the significance of these NGOs' organizational culture to gender transformation in a wider society.

\subsection{Significance of the research}

The significance of this study is reflected in a couple of dimensions as follows. First, I expect to address a gap in existing knowledge about how NGOs in Vietnam are implementing gender mainstreaming in practice. From there, I can see what opportunities and constraints face them in their work. Secondly, the research involves useful organizational case studies through which I have been able to assess important gaps between theory and practice. These gaps have implications for efforts to address on-going gender inequality.

\subsection{Theoretical and methodological approach}

In this research, I used a feminist epistemology approach to investigate the current implementation of gender mainstreaming in NGOs based in Vietnam. This approach is chosen because it stresses on experiences of women and social construct influencing gender relationships and dynamics. As such, women become starting points while gender is taken as central to how it understands the production of knowledge. Using a feminist epistemology in research helps to ensure equal power sharing between the research and its subject(Harding, 1987). To allow analysis of specific views on NGOs' gender work in office and field, qualitative methods were used in this study because they offer direct and spontaneous sharing of information, local knowledge, expertise, and experiences between researchers and informants (Mikkelsen, 2005). This enables researchers to generalise, explain and draw 
conclusions from the data. Qualitative case studies were also employed to learn about and uncover the complexities of each case as a whole. Semi-structured interviews were the principal technique to collect data for the research. The participants of the research included one INGO and one LNGO. Originally two INGOs and two LNGOs were chosen, however insurmountable obstacles prevented all these from being involved in the study. By choosing two organizations, I was able to carry out comparative case study research.

\subsection{An overview of the research}

Figure 1.1 provides an overview of how this thesis is structured. Chapter 2 comprises a literature review covering the basic feminist theories relevant to approaches tackling gender inequality and the concept of gender mainstreaming and its adoption in the development sector including NGOs. Chapter 3 outlines the predominantly quality methods of the study including desk reviews, participant observation and semi-structured interviews. It also discusses forms of analysis. Chapter 4 portrays the traditional ideology of gender roles and its changes over time as a result of economic reform in Vietnam. Chapter 5 looks at how the idea of gender mainstreaming has been translated into practice in the INGO and the challenges encountered in implementing the idea effectively. Chapter 6 examines the same issues but in the LNGO. Chapter 7 concludes with a discussion of the results and with recommendations for future research. 
THESIS OUTLINE

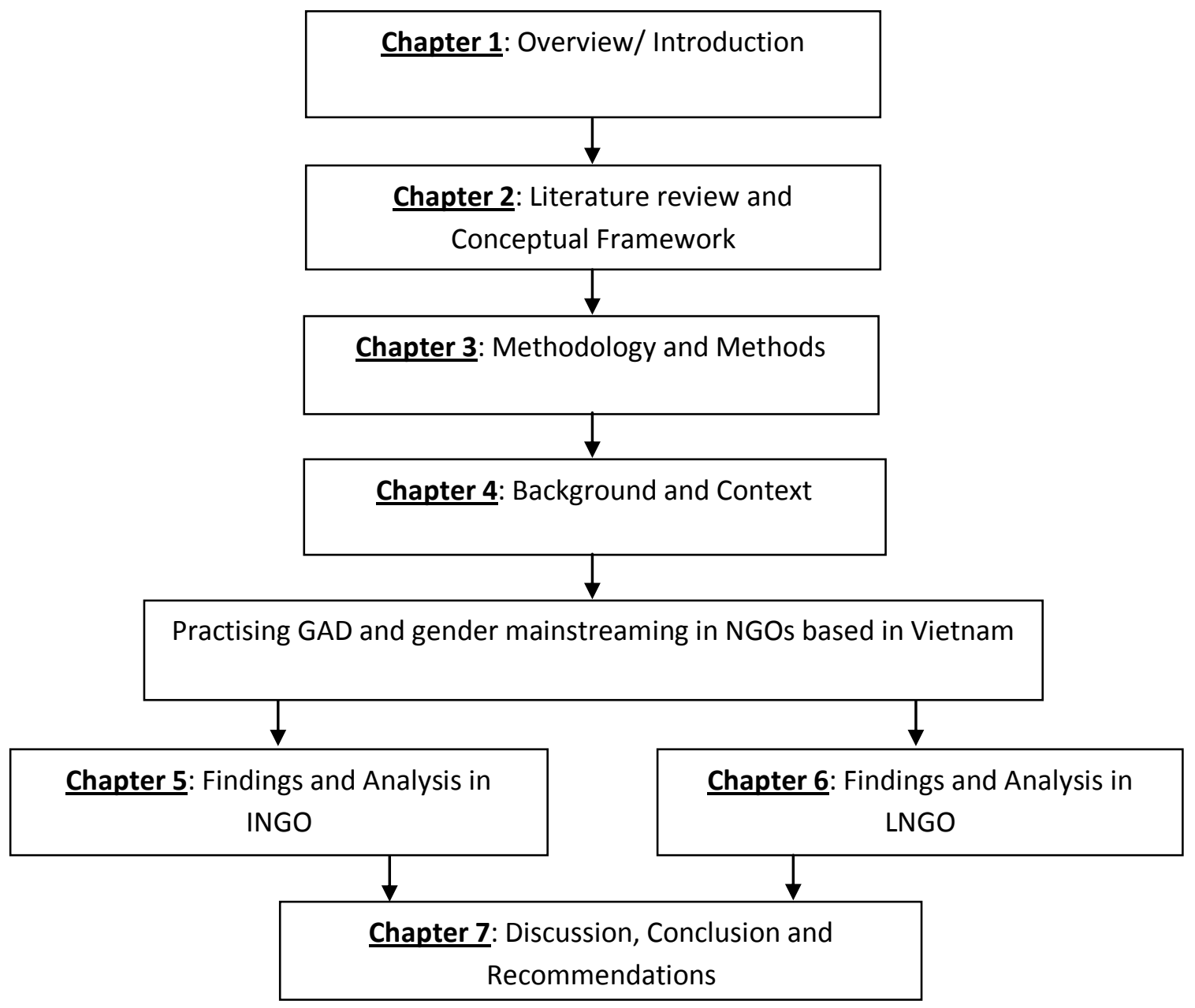

Figure 1.1. Thesis outline 


\section{Chapter 2:Theoretical framework and literature review}

\subsection{Introduction}

What is a domesticated woman? A female of the Species ... She only becomes a domestic, a wife, a chattel, a playboy bunny, a prostitute or a human dictaphone in certain relations. Torn from these relationships she is no more the help-mate of man than gold in itself is money.

(Rubin, 1975: 158 cited in Razavi \& Miller, 1995:12)

Gender inequality has been a persistent global issue. Its removal requires a long-term and consistent commitment by all concerned, including theorists, agencies and individuals. Since the UN launched the Decade for Women in 1976, countless interventions and attempts have been made to promote gender equality in most societies at international, national and local levels. In this literature review, I present four main sections. In the first, I review different approaches or feminist theories to gender equality. Gender mainstreaming as a popularly adopted strategy is included. Second, I provide a brief summary of international frameworks to address gender inequality. Then, I discuss the rise of NGOs and how these organizations are involved generally in development work and in gender equality promotion in particular. Lastly, I have a deeper look at why academics are sceptical about the success of gender mainstreaming as this aspect will be the central theme of the thesis.

\subsection{Review of different approaches or feminist theories to gender equality}

\subsubsection{Women in Development (WID)}

The term 'women in development' (WID) was first introduced in the early 1970s by the Women's Committee of the Washington, $\mathrm{DC}^{1}$. The year 1975 was a milestone for WID when the UN launched the Women's Decade (1976-1985)(Ostergaard, 1992). According to Moser (1993), this decade strongly emphasized the importance of women's role in socio-economic development, a role that had been invisible. Overholt et al (1984, p. 3 cited in C. Moser,

\footnotetext{
${ }^{1}$ 'Chapter of the Society for International Development, network of female development professionals who were influenced by the work on Third World development undertaken by Ester Boserup and other 'new' anthropologists' (Boserup, 1970; Tinker, 1982 and Maguire 1984 cited in Moser, 1993)
} 
1993, p. 2)stated that 'women are key actors in the economic system, yet their neglect in development plans has left untapped a potentially large contribution'. It seems that this analysis could be traced to Boserup's (1970) book “Women's Role in Economic Development" in which data and evidence was used to show women's traditional role and main contribution to agricultural production (Boserup, 1970 cited in Razavi \& Miller, 1995). The rationale of such an analysis is that the exclusion of women from economic systems could be attributed to the difference in status and power between men and women(Razavi \& Miller, 1995).

Moser (1993) has identified a fivefold schema for different approaches to addressing gender inequality through the WID framework. These are: "WID-Welfare", "WID-Equity", "WIDAnti-poverty", "WID-efficiency" and "WID-empowerment". WID-welfare gained popularity in the 1950s and 1960s. This particular WID approach focused on how women in 'developing' countries could be integrated into 'development' processes and therefore become better mothers. Women were viewed as passive recipients of 'development' projects so that they could better undertake their reproductive role(C. Moser, 1993). It was subsequently argued that women's contribution to production should be also strongly acknowledged. The notion was supported by Ostergaard's point (1992) that while 'the biological nature' of women is to bear and rear children, this should not be understood as their natural obligation to domestic activities. This false stereotype leads irrationally to the treatment of women as a minority group even while they make up more than half of the human population. Yet WID-Welfare was not really a WID approach compared with subsequent WID approaches, as it did not seek to change conditions for women. This helps explain the need for a more holistic approach to the conceptualization of women's issues(Ostergaard, 1992).

WID-equity aimed at gaining equity for women through the recognition of the triple role (productive, reproductive and communal) played by women. This approach also sought to meet strategic gender interests and challenge women's subordination through direct state intervention so that women would be given political and economic autonomy and accordingly become more equal with men (C. Moser, 1993). It can be seen that WID-equity did attempt to make structural changes. Meanwhile, WID-Anti-poverty viewed poverty as 
the cause of women's inferior position and underdevelopment, hence its intervention sought to enhance women's productivity. Understandably, projects under this schema mainly focused on providing women with small-scale and income-generating skills. WIDAnti-poverty was complemented by the subsequent approach, WID-efficiency, which again recognized women's economic contribution to effectiveness and efficiency of development process (C. Moser, 1993). It is worth noting that the emergence of the last approach, WIDempowerment, was a benchmark for progress in addressing women's concerns. This approach asked women what they needed and let them decide on their own. Generally speaking, WID-empowerment acknowledged women's relationships with men through its emphasis on equal-power-sharing and equitable partnerships between women and men. The goal of this approach was to transform unequal gender relations in political, societal and economic dimensions. In so doing, it was thought that sustainable and people-centred development would be ensured (C. Moser, 1993).

Although WID initiatives managed to make women more visible in policy design and implementation as active agents in development process, they also had some shortcomings. Firstly, WID generally concerned the problems of women themselves in isolation from men(C. Moser, 1993). WID was grounded in traditional modernization theory that focused on macroeconomic development. This was reflected in the tendency to include skill training for women in all WID projects because of a belief that women would no longer be subordinate to men if they gained economic independence. However, this leads to a situation whereby 'women must perform two roles in society while men still perform only one', rather than the emancipation of women. Tinker (1976, p. 3 cited in C. Moser, 1993)claimed these projects actually increased the inequity between men and women. Secondly, WID was based on a liberal feminist approach but did not advocate structural change. While it did not question the root causes of gender inequality it also missed the impacts of class, race, and culture(Rathgeber, 1990). Finally, it appeared that WID focused on the problems of women in terms of sex, rather than gender difference (C. Moser, 1993) formulated by 'ideological, historical, religious, ethnic, economic and cultural determinants' (Whitehead, 1979 cited in C. Moser, 1993, p. 3). Consequently, the unequal social and power relations between women and men remained unaddressed (Elson, 1993a, p.5 cited in Razavi \& Miller, 1995) 


\subsubsection{Women and Development (WAD)}

Women and Development (WAD) emerged in the second half of the 1970s. It was grounded in a neo-Marxist feminist approach and sought to address the limitations of modernization theory within WID (Rathgeber, 1990). The WAD approach made a small improvement by focusing on the relationship between women and the development process, while recognizing that men were also adversely affected by the existing social structure of inequality (Rathgeber, 1990). It has been argued that the WAD framework offered a critical analysis of how gender inequality is constructed but offered no functional alternative, failing to analyse the relationship between the various forms of society, patriarchy, different modes of production, and women's subordination (Rathgeber, 1990). It did not, therefore, address the question of how a given society systematically under-represented women. Rather it focused on solving women's problems by more thoroughly designed intervention strategies; it did not bring about change in the social relations between men and women (Rathgeber, 1990).

\subsubsection{Gender and Development (GAD)}

The discontent and limitations of WID/WAD stimulated the use of Gender and Development (GAD) in the 1980s, which had theoretical roots in socialist feminism. According to Rathgeber (1990), this approach dealt with the shortcomings left by modernization theory in the way that it linked the relations of women's productive role to that of their reproductive role. At the same time, all spheres relating to women's lives were starting to be taken into consideration in order to bring about full equality for women within the framework of the development process (Braidotti, Charkiewicz, Hausler, \& Wieringa, 1994). The assumption of GAD identified women's inferior status as a result of the social construction of production and reproduction. Hence, GAD raised the question of why women were systematically subordinate. Young (1987 cited in Rathgeber, 1990) contributed some critical points to the GAD perspective, which was believed to go further than the two previous approaches. Firstly, GAD did not concern women in isolation but looked at the social construction of gender relations. Secondly, it went deeper into the specific roles and responsibilities performed by men and women in a given society. Thirdly, men were encouraged to make contributions to addressing the issue of equity and social justice. Finally, the GAD approach 
emphasized both the productive and reproductive roles of women by considering their contributions inside and outside the household. According to Rathgeber (1990), one feature that made GAD distinctive was that the so-called private sphere was opened up to see how the relationship between men and women was constructed. More importantly, socialist feminism also raised the idea of a strengthened role for the State in providing women with social welfare and for women's voices to be heard in politics. In doing so, GAD sought to transform unequal gender relations to enable long-term sustainable development for both women and men(Miller, 2009).

As Rathgeber (1990) concluded, GAD aimed not only to lead to better-designed intervention and strategies but also to better integrate women into contemporary development attempts. It also strived for structural and power change in given societies to empower women at both national and international levels. In some cases, the GAD approach has been adopted simply to reassure men that there is not an excessive focus on women, rather than as a commitment to improving women's status. For example, in 1994, the World Bank applied the GAD approach as a framework to design its own gender policy but in practice only dealt with women in isolation; women's priorities and needs within the specific contexts where men - an obvious and important component were not identified(C. Moser, Tornqvist, \& Bronkhost, 1999).

In fact, men and women experience power differently and unequally so power necessarily becomes a part of any analysis of gender relations. Unequal power relations are just one of the many gender dynamics that are very important for personal and organizational change. Gender inequality must be understood as connected with other inequalities such as race and class(Plowman, 2003). Moser (1993 cited in Plowman, 2003)notes that the GAD theory and practice have become predominant in mainstream development with a move away from a focus on external programming and instead focused on planning to get one's own house in order first. As a result, GAD has been firmly established at the Beijing Platform for Action as a foundation to bring about gender equality. Yet, in practice the confusion about its distinction from WID remains pervasive (Miller, 2009) and translating this theory into practice is not easy task. 


\subsection{Gender Mainstreaming}

\subsubsection{Definition of Gender Mainstreaming}

As feminists continued to influence mainstream development, gender mainstreaming became fundamental to the application of Gender and Development(Subrahmanian, 2009). At the UN Conference on Women held in Beijing in 1995, governments across the world unanimously agreed to prioritize gender mainstreaming as an important mechanism to achieve the goal of gender equality. As a result, a great deal of support from international institutions was poured into governments and civil society to carry out this process(A. Moser \& Moser, 2005). Gender mainstreaming is defined as a strategy for institutionalizing and integrating gender concerns into the mainstream to address the critique that the legal, social, and economic rights between men and women are unequal (A. Moser \& Moser, 2005; Tiessen, 2007). Gender equality is understood as recognition of different needs and priorities between the two sexes so that both should have equal opportunities to fulfil their rights and participate in national, political, economic, social, and cultural development (CIDA, 1999 cited in A. Moser \& Moser, 2005). The concept of gender mainstreaming is embodied in a comprehensive definition by the UN Economic and Social Council (ECOSOC) in 1997 (A. Moser \& Moser, 2005; Porter \& Sweetman, 2005; Tiessen, 2007) as follows:

Mainstreaming a gender perspective is the process of assessing the implications for women and men of any planned action, including legislation, policies or programmes, in all areas and at all levels. It is a strategy for making women's as well as men's concerns and experiences an integral dimension of the design, implementation, monitoring and evaluation of policies and programmes in all political, economic and societal spheres so that women and men benefit equally and inequality is not perpetuated. The ultimate goal is to achieve gender equality (cited in A. Moser \& Moser, 2005, p. 12) 


\subsubsection{Critical insights into waysof gender mainstreaming}

Jahan (1995) identified two ways of mainstreaming gender into policy agenda including integrationist and agenda-setting. The former approach is thought to have started during the UN Decade for Women 1976-1985 and aimed at integrating discussion of gender issues into the relevant sections of agency documents. This form of mainstreaming is a response to the status of male privilege and the marginalized status of women's concerns as pointed out in WID(Kabeer, 1994). In other words, it aims to address the practical needs of women. This approach has been dubbed (and criticized) by feminists as an 'add women and stir' approach(Porter \& Sweetman, 2005, p. 2). More recently, a transformative or agendasetting approach has aimed to 'transform an existing development agenda from a gender perspective' (Jahan, 1995, p. 216) to address strategic needs, starting from a gender analysis of the inequalities between men and women, whereby unequal gender relations are understood as intersecting with other inequalities such as race and class (Porter \& Sweetman, 2005).

Elson (1992 cited in Kabeer, 1994)believed that such a transformative approach required a different way of thinking and an alternative way of doing, ways which went beyond seeking to include gender issues in the development agenda, but instead enabled women to have a greater role in setting the agenda. The goal of this approach is politically ambitious in the way that it means to change the rules, rather than play by them(Kabeer, 1994). Unfortunately, even this transformative agenda for gender equality has been criticized for stopping at the level of technical strategies, rather than creating political ones (Kabeer, 1994; Tiessen, 2007).

Integration and transformation happens at two different institutional levels. Integration involves working with development institutions while a transformation approach seeks constitutional change. Therefore, the latter requires a deep understanding of the nature of politics, society-state relationships, the context of policy-making institutions, and the influence of international development and financial institutions. In practice, the relationship between integrationist and agenda-setting approaches has been interrelated in order to mainstream gender equality concerns in development. Put another way, 
transformation depends on integration for its success(Mukhopadhyay, 2004).Mukhopadhyay (2004) points out the requirement for both approaches to explicitly acknowledge equality goals entailing the redistribution of power, resources and opportunities in favour of the disadvantaged. In order to achieve transformative goals, Mukhopadhyay (2004) suggests looking at a number of aspects ranging from 'policy reform, advocacy, capacity building, analytical frameworks, programme development, monitoring systems', and analysis of the pros and cons of each aspect(Subrahmanian, 2004 Rao \& Kelleher, 2005, p. 63). (See Figure

\begin{tabular}{|c|c|c|}
\hline $\begin{array}{ll}\text { Informal pressures } \\
- & \text { Ideology } \\
- & \text { Culture } \\
- & \text { Unequal power } \\
& \text { relations }\end{array}$ & $\begin{array}{l}\text { Social and public } \\
\text { accountability } \\
\text { - } \quad \text { Economic and political } \\
\text { opportunities } \\
\text { - } \quad \text { Equity and inclusion } \\
\text { - } \quad \text { Decentralization of } \\
\text { resources } \\
\text { - } \quad \text { Transparency and } \\
\text { governance } \\
\text { Social change } \\
\text { Interaction between } \\
\text { institutions, people, } \\
\text { processes, structures } \\
\text { Mobilisation and voice } \\
\text { Rights and choice } \\
\text { Capabilities, assets, } \\
\text { resources } \\
\text { Empowerment }\end{array}$ & $\begin{array}{l}\text { Formal pressures } \\
\text { - } \quad \text { Leadership and vision } \\
-\quad \text { Gender equality on } \\
\text { the agenda } \\
\text { - } \quad \text { Inclusive structures } \\
\text { - } \quad \text { Effectiveness } \\
-\quad \text { Accountability }\end{array}$ \\
\hline
\end{tabular}

Figure 2.1. Dynamics between top-down and bottom-up forces of change (Rao and Kelleher 2005: 63)

Moreover, gender mainstreaming is also defined by DFID as a twin-track approach whereby the integration of both sexes' concerns into the development process is regarded as one component and specific activities targeted at women's empowerment as the other. The eventual outcomes of this strategy are increased equality and empowerment(C. Moser, 2005). This strategy is better demonstrated in the following figure: 


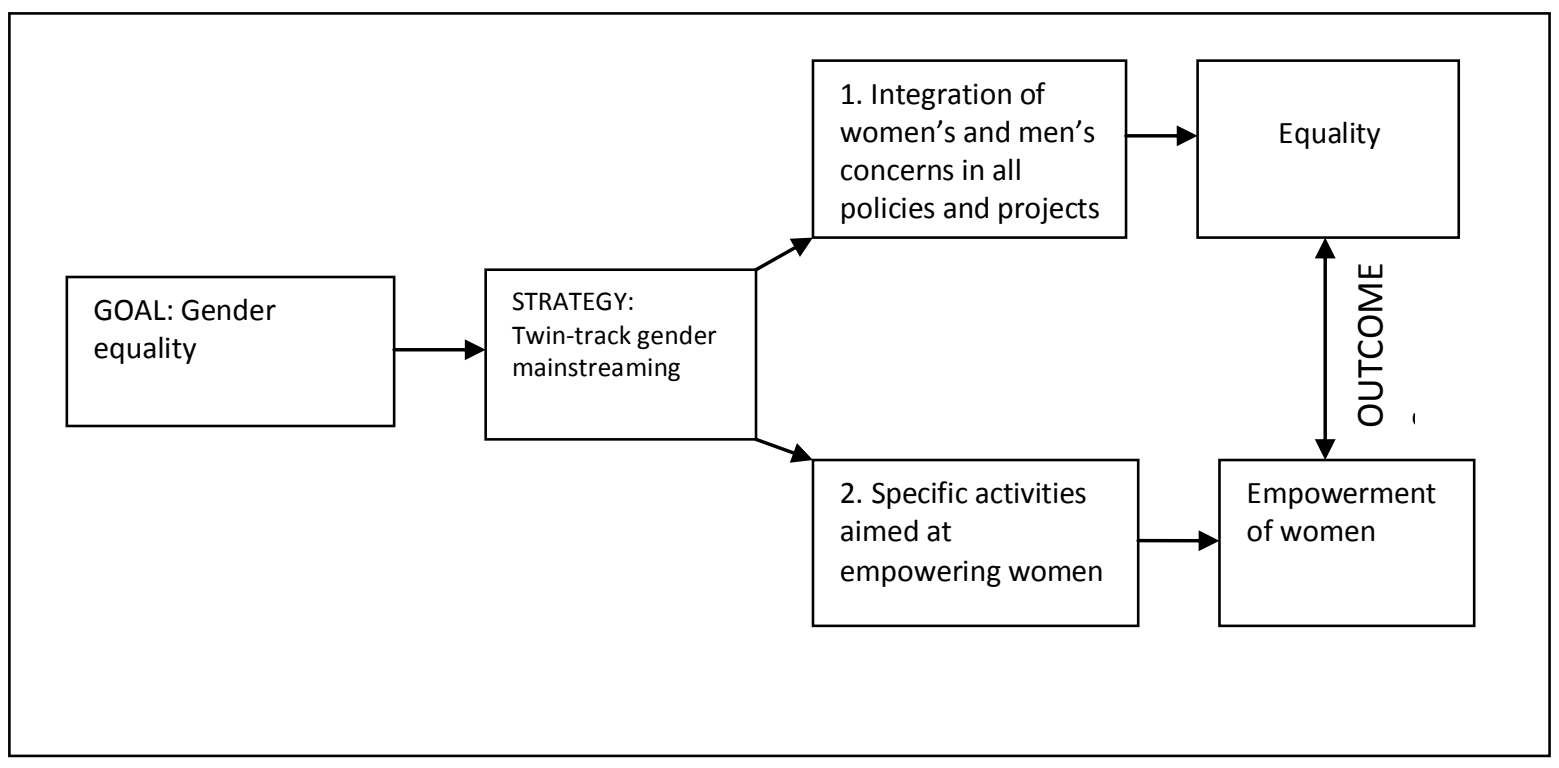

Figure 2.2. DFID gender mainstreaming strategy (C. Moser, 2005, p. 582)

\subsection{International institutional frameworks on gender equality}

At the international level, gender issues have drawn enormous attention as one of the top priorities in efforts to combat hunger and poverty. The principle of gender equity was stated first in the Universal Declaration of Human Rights over 50 years ago. In this Declaration, all human beings are entitled to basic rights and freedoms regardless of what sex they are. They are born with equal dignity. This principle has resulted in closing some of the specific gaps in international human rights on gender equity(Wells \& McEwan, 2004).

Gender has been integrated into the Millennium Development Goals (MDGs). These goals were agreed on by 180 countries in the world in 2000 and have eight goals in total, specified by a series of the targets and indicators aimed at poverty elimination by 2015 . Although gender concerns cross all goals, Goal \#3 particularly and directly addresses gender equality and women's empowerment. The MDGs provide an important framework for development institutions to work together and evaluate progress(Miller, 2009). In MDGs and other development goals, it is crucial to make sure that gender equality goals are targeted and monitored by aid structures. Eventually, the outcomes of gender equality will contribute to the effectiveness of aid delivery(UNIFEM, 2006), or, in the words of the 1995 Human 
Development Report, 'development, if not engendered, is endangered'(UNDP, 1995 cited in Tiessen, 2007, p. 14).

In addition, other international instruments have been adopted to conceptualize the human rights of women and integrate them into a mainstream human rights framework. These instruments are the Declaration on the Elimination of All Forms of Discrimination against Women in 1967 and the Convention on the Elimination of All Forms of Discrimination against Women (CEDAW) in 1979 adopted by the UN. These documents have been recognized as representing a major turning point in international human rights law but also as effective tools which go beyond legal documents to address inequality between men and women(Ali, 2008). In 1995, the Beijing Platform for Action was signed by representatives of 189 governments, which, like CEDAW, identifies a list of areas of special concern to women (A. Moser \& Moser, 2005). Recently, the Paris Declaration and the Accra Agenda for Action (2008) provide the frameworks and good practice principles to take gender equality as a priority development issue.

These theoretical and institutional frameworks became the basis for gender work in development institutions, including NGOs, the primary participants within the scope of this thesis. The following section will be the history of NGOs in development work and their engagement in gender work.

\subsection{Non-governmental organizations and gender work}

\subsubsection{The history of NGOs and the rise of their involvement in development work}

Development as we know it today is believed to have been launched by President Truman in 1949 and was accompanied by the birth of UN agencies(Pearce, 2000). The World Bank realized the importance of NGOs as an important force in the development process(AusAID, 1995). NGOs are thus key to my discussion of alternative development and the theoretical justification for their activities including gender mainstreaming.

The original NGOs became visible in the 1960 s as welfare or emergency relief agencies, but were very small in both size and number, and mostly based in the global north (Eade, 2000). 
AusAID (1995) defined NGOs as non-profit, non-governmental organizations undertaking or supporting the international development community, of a distinctive legal character and concerned with public welfare goals. NGOs targeted groups of poor people who were often lacking of support (Lewis, 2005 cited in Bebbington, Hickey, \& Mitlin, 2008). NGOs mainly deliver socio-economic services or policy advocacy. They place people at the centre of development, which frequently challenges governments (Clarke, 1998; Desai, 2005).

As alternative agents to development, NGOs have proliferated recently, both in developed and developing countries. The number of international NGOs has increased from fewer than 200 in 1909 to nearly 1000 in 1956 and then to over 20,000 in 2005(Union of International Association, 2005 cited in Werker \& Ahmed, 2007). They accounted for US\$9 billion of aid to developing countries in 1993 (Smillie \& Helmich, 1993 cited in AusAID, 1995). To promote international development assistance, high-income countries have increased their funds to NGOs from a negligible amount to nearly \$2 billion in 2004 (OECD, 2006a cited in Werker \& Ahmed, 2007). The role of NGOs in addressing poverty and hunger has become more significant than ever before thanks to their comparative advantages over the state mechanisms in a number of activities (Brinkerhoff, Smith, \& Teegen, 2007; Clarke, 1998).

Their greatest strengths are around advocacy and using participatory models of development that put human development at the heart of their operations. NGOs effectively strive to illustrate that poverty can be tackled by having project beneficiaries take part in the planning, implementation and sustainability of projects (Chege, 1999). A working paper issued by the UN Industrial Development Organization (UNIDO) summarizing NGOs' comparative advantages, included local accountability, independent assessment of issues and problems, expertise and advice, reaching important constituencies, dissemination of information, and awareness-raising (Chege, 1999).

NGOs bring innovation in programme design and implementation into close contact with the poorest of the poor; flexibility in programme structures and independence from public policy; strength in technical expertise and knowledge; the ability to mobilize public participation and resources; better understanding of the needs of the poor and communities; public trust and credibility(Brinkerhoff et al., 2007); and a high degree of 
people's involvement and participation in their activities (Chege, 1999). Many NGOs are viewed partly as watchdogs and advocates for human rights and good governance.

NGOs have also become more important in the development sector because they often address issues that governments are unable or unwilling to take up through their efficient, innovative and cost-effective approaches to tough social and economic problems. In this sense, they are able to reach into spheres where governments are constrained by bureaucratic or political considerations. Another strength of NGOs is a growing relationship with the UN and its agencies through which they are able to discuss and formulate policy recommendations on social and economic issues.

In fact, every UN agency cooperates with NGOs. For instance, the UN High Commissioner for Refugees has some 400-500 partners and, in 1997, 272 million USD in funds channelled through 443 NGOs in 131 countries. Further, the World Bank also has a portfolio of projects approved each fiscal year clearly indicating NGOs' involvement (Chege, 1999). The share of the World Bank projects with civil society's involvement, including NGOs grew from six percent in the late 1980s to over 70 percent in 2006(the World Bank, 1995; 2006a cited in Werker \& Ahmed, 2007), indicating NGOs' increasing political influence.

The twentieth century witnessed the shift from 'top-down' to 'bottom-up' approaches to social and economic development (Eade, 2000; Kamat, 2004; McGee, 2002). Ideally, involving beneficiaries - such as women -in development process is a way to empower them, so that they are able to take control over their own lives and determine their own development. The most notable example is found in the work of many development-minded NGOs (McGee, 2002; Rowland, 2003). NGOs have employed this participatory approach to address root causes of poverty(Chambers, 1997; Clark, 1991; Gardner \& Lewis, 1996 cited in Kamat, 2004)

NGOs work with communities at the grass-root level and meet the immediate needs of the beneficiary, characteristics seldom found in government projects. NGOs thus contribute to protecting the interests of minority groups in a given society while demanding governmental accountability to the public (Smillie \& Helmich, 1993 cited in AusAID, 1995). They are viewed as effectively participatory implementers of programmes in fields of health care, literacy, 
and small-scale income generation projects (Bebbington \& Farrington, 1992; Clark, 1997 cited in Kamat, 2004).

Despite the positive outcomes NGOs have brought to the development field, especially in inclusive participatory development, they have also faced criticisms. They have been criticized for being as corrupt and undemocratic in practice as State governments (Gibbon \& Bangura, 1992) and are frequently found to be donor-dependent and subject to 'personal rule' (Ndegwa, 1996). Also, they may be: representative of elite interests; incapable of, or plainly uninterested in, political lobbying; and fragmented, unorganized, and competitive (Fowler, 1993; Mercer, 2002, 2003; Mohan, 2002). In this way, NGOs, if not in good hands, may side-line one or two social groups such, for instance, as women.

\subsubsection{Gender mainstreaming in global NGOs}

Gender mainstreaming has been visible in a variety of development organizations ranging from donors to NGOs since the Fourth World Conference on Women, held in Beijing. Governments have increasingly adopted this tool to incorporate gender issues in diverse sectors and bring gender equality to the centre of all development activities (Tiessen, 2007). In theory, gender mainstreaming provides opportunities for women and men to work for gender equality in their organizations, programs, and projects.

Within this, NGOs are active players in using gender policies to shape their functioning and projects. The World Bank defines NGOs as 'private organizations that pursue activities to relieve suffering, promote the interests of the poor, protect the environment, provide basic social services, or undertake community development' (the World Bank, 2002 cited in Tiessen, 2007, p. 21). Manderson\& Mark (1997) say that NGOs are better than other actors in working at the grass-root level and dealing directly with the local beneficiaries. NGOs therefore are known for addressing inequality (including gender) more effectively on the small scale than governments. As Dema (2008)contends, NGOs are more conducive to reproducing or transforming gender relations.

Gender has been institutionalized within NGOs via the gender norms, attitudes and practices of their staff and systematically incorporated into the policies, strategies and procedures 
that shape their development work and functions (Manderson \& Mark, 1997; Tiessen, 2007; Wallace, 1998). Researchers and practitioners have conducted research and surveys on gender mainstreaming using NGO samples in different countries. For example, Oxfam GB in the South America has a focal gender point staff and considers gender work as a concern or responsibility for all staff, rather than just a few specialists. In this organization, gender is taken into consideration as part of all organizational policies and procedures. Staff are required to be aware of Oxfam's gender strategy and have the capacity to carry out gender analysis to ensure that all staff can sensitize gender issues to their work (Wallace \& Wendoh, 2005).

Gender relations are frequently and thoroughly analysed and incorporated in all stages of planning, implementing and evaluating programmes (Dawson, 2005; Wallace \& Wendoh, 2005). In Malawi, for instance, many NGOs include gender in various activities, such as employing more female staff, providing training workshops on gender issues for staff and making policies to improve gender equity (Tiessen, 2007). Australian NGOs working overseas emphasize the bottom-up approach to give poor women full ownership of and responsibility for all project activities in Zimbabwe and India(Desai, 2005; Osirim, 2001; Wallace \& Wendoh, 2005).

\subsection{Gender Mainstreaming in practice: Success or Failure?}

While there have been important steps forward for gender equality, the real changes these policies and initiatives have brought are ambiguous (Dawson, 2005; Desai, 2005; Wallace \& Wendoh, 2005). To prove this point, a decade after the UN conference, an evaluation on progress on gender mainstreaming was conducted. The result of this review led to a wave of scepticism as to whether or not gender mainstreaming works as an effective tool in practice (Jahan, 1995; Kabeer, 1994, 1999, 2005; A. Moser, 2007; A. Moser \& Moser, 2005; C. Moser, 2005; Tiessen, 2004, 2007). It is very much likely that the failure of gender mainstreaming is caused by the following constraints: 


\subsubsection{Lack of a conceptual clarity around the key terms}

Doing gender work requires people first to fully and correctly understand some key terms including gender and gender mainstreaming. However, these terms are problematic in themselves, causing confusion to people.

\section{a. The confusion between gender and sex/gender and women}

Sex refers to biological characteristics which differentiate the male and the female of the human species. Due to this biology, both sexes need food, water, sleep, clothing and shelter for their basic survival and existence. Meanwhile, gender refers to social-cultural constructs including norms, values, customs, and practices through which the roles, responsibilities, capabilities, attitudes, and behaviours of men and women are determined. Simply speaking, gender can be seen as a social and symbolic construction which defines what people imagine a man or a woman to be and as a social relationship through which people expect a man or a woman to behave and act (Miller, 2009). However, there is a lack of conceptual clarity around what is meant by gender. As a consequence, people often use gender as a form of shorthand for 'women' or 'men and women', or even 'sex' (Babacan, 2004; O'Neil, 2004).

The lack of understanding of gender as a concept allows people to easily conflate gender with sex (Kabeer, 1999). On the one hand, it creates a common assumption that gender relations are natural and biological and cannot be changed (Kabeer, 1999). In practice, gender is often unintentionally or intentionally thought to only refer to women but gender more appropriately refers to transforming unequal social relations between men and women in terms of access to power and resources, which lead women to be less valued than men in a given society. In this area, Kabeer (1994) notes that the subordinate position of women to men is not natural but constructed by societies in which men have been privileged by particular influential factors such as culture, tradition and religion. Plowman (2003, p. 106) also asserted that "gender inequalities are not set in stone and they can be changed". In some other cases, using the terminology of gender can render women invisible(Rea, 2004). Such confusion brings the whole activity into question. As a result, 
people fail to understand all the issues with gender mainstreaming, let alone those facing the implementation of the strategy (Mitchell, 2004).

\section{b. Between gender and development and mainstreaming women}

The failure of gender mainstreaming can also be blamed for the on-going confusion between gender and development and mainstreaming women. For some, gender mainstreaming means no more than emphasising the role of women and having more women in the organization's human resource base. In theory, gender mainstreaming does not exclude specific intervention directed towards women. Focussing on women helps crucial progress towards gender equality which is considered to be ultimate goal of the gender mainstreaming process (Mukhopadhyay, 2004; Rao \& Kelleher, 2005). In practice, however, the focus on women has come to signify the opposite. It has frequently led to cases such that when budget cuts occur, gender mainstreaming is cut or reduced (Mukhopadhyay, 2004). This situation can hardly be improved because the agenda is often driven by outside agencies as a part of conditionality for aid.

Another misunderstanding here is that gender equality would be achieved solely by increasing the number of women in the organization and in positions of decision-making. This idea is pushed by well-meaning donors who include the notion in checklists designed to measure whether client governments or NGOs have progressed towards gender equality. This is a straightforward measurement compared to integrating gender analysis in the formulation of policies, programmes, and projects. Although it is important to provide women and men with equal opportunity, this single measure "cannot be the be-all and endall" (Mukhopadhyay, 2004, p. 99). If this indicator is introduced in an ahistorical and decontextualized manner, the organization may face the serious consequences of gender politics. In this sense, some explain the increase in the number of women in their organizations as a way of constituting gender mainstreaming. They are resisted by other colleagues who believe that hiring more women leads to poor working performance. This thought is derived from viewing women as unqualified and reinforced by a dominant culture of misogyny (Mukhopadhyay, 2004). 


\section{c. Men and development}

The trend emphasising women in isolation risks excluding men from the process. Gender equality has been widely seen as the concern of women, not men, as women are disadvantaged by gender inequality. Women started to place gender issues on agendas in gender and development work, thus - according to some thinking - gender issues are of no concern to men. However, this logic has been challenged by those who float the idea that gender are inevitably issues of men and boys as well(Connell, 2003, p. 3 cited in Flood, 2004). Since this thought has been introduced, the inclusion of men in efforts towards gender equality has been rapidly evolving in the philosophies and programmes of international organizations. The significance of male involvement is widely recognized because "men are both part of the problem and part of the solution"(Flood, 2004, p. 27).

There is a need for change in men's attitudes and behaviours because they, in many cultures, play a crucial role as decision makers and community leaders. The patterns of gender injustice are tied to social constructions of masculinity and male identity whilst there is an intimate link between gender inequality and men's practices and identities. Men's participation in complex and diverse gender relations and masculine discourses and culture can promote gender equality. At the same time, Flood (2004) cautions that the inclusion of men risk reinforcing existing power structures and limiting resources and funds that could be directed towards women (Kaufman, 2003, p. 5 cited in Flood, 2004). In reality, some men show resistance while others show support for gender equality. It is necessary to recognize this diversity so as to have different strategies to respond to resistance while mobilizing their support. Moreover, men also pay significant costs, particularly to men's emotional and physical health as a consequence of gender inequality. Thus, it is also worth recognizing that some men already are playing a role in fostering gender equality, rather than seeing them only as obstacles to women's empowerment (Flood, 2004).

\subsubsection{The lack of political will for institutional change}

It is now believed that the root causes of gender inequality need to be addressed by institutional change (Mukhopadhyay, 2004; Rao \& Kelleher, 2005; Standing, 2004; 
Woodford-Berger, 2004). The institutional change cannot occur in the absence of strong political commitment. The technical tools such as gender sensitivity trainings, gender analysis, and so on are insufficient to transform the male-dominated mainstream(Corner, 2004). It is evident that the increased number of women in decision-making bodies and political office does not always guarantee support for the advancement of women in general. Hence, the lack of political will has been a major obstacle to effective implementation of gender mainstreaming(Corner, 2004).

Institutional change would most sustainably be made through changes to stated/formal and the unstated/informal rules. The formal rules include laws, policies, school curricula and so on, while the informal ones include inter alia, cultural arrangements and norms (Rao \& Kelleher, 2005). There is an obvious assumption here that change at one level will automatically lead to change at others. Rao and Kelleher (2005) prove that it is not always the case. For example, when women gain economic independence, their financial status cannot be enough to protect them from domestic violence. Additionally, Corner (2004) claims that women become as a tool for women-specific projects to achieve other policy ends such as fertility control, efficiency in the use of development resource and so on. The World Bank states that "investing in women can be a cost effective route to economic efficiency" (the World Bank, 1989, p. iv cited in Corner, 2004, p. 17). Therefore, gender inequality can only be redressed if both formal and informal interventions occur. Doing this requires development organizations' willingness and ability to intervene in a significant way as illustrated in the figure below: 


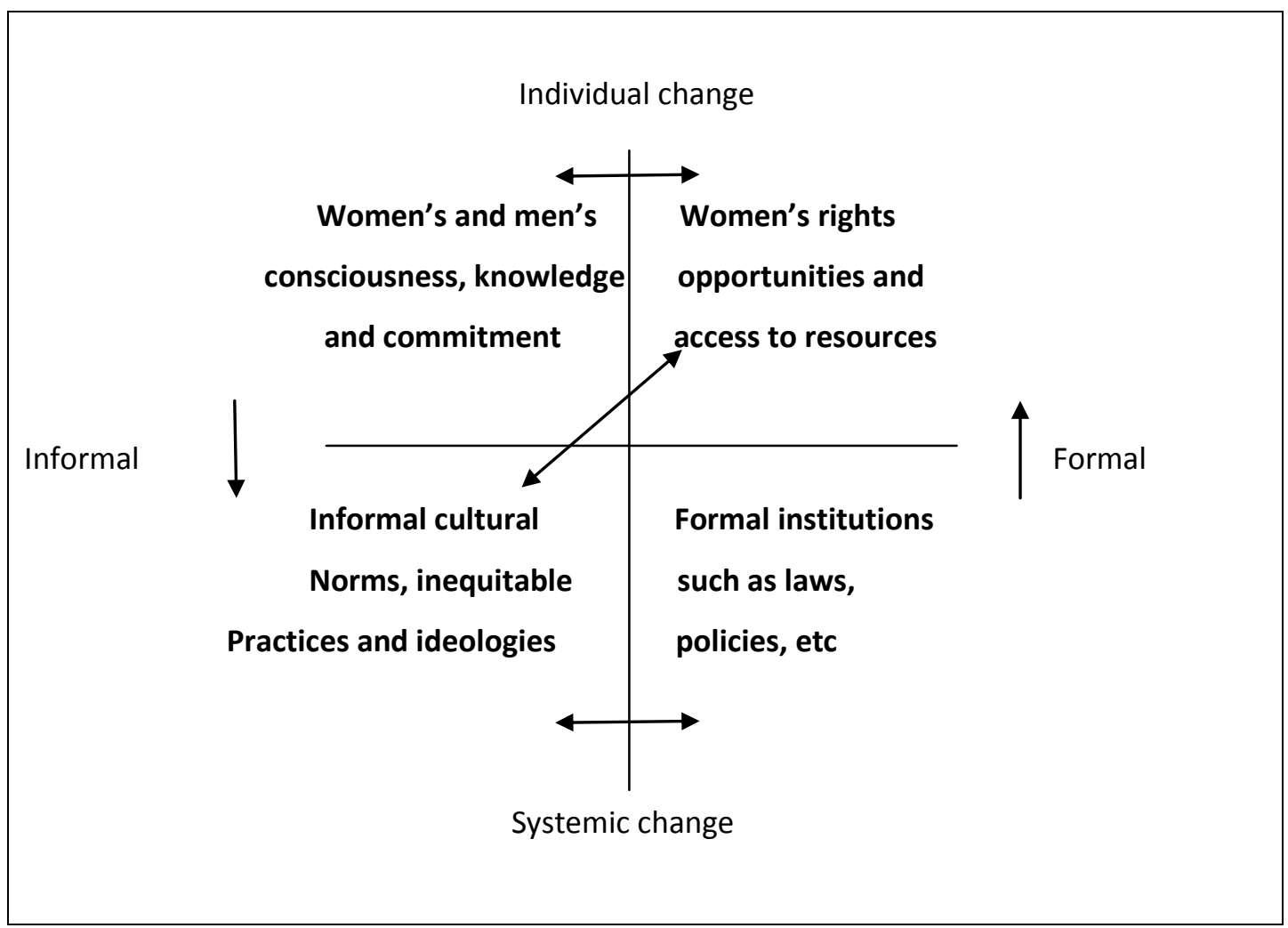

Figure 2.3. Gender at work: what are [organizations] trying to change? (Adapted from Rao and Kelleher, 2005, p. 60)

Institutional reforms may be initiated but restricted to fiscal and administrative reforms, rather than dedicated to making systems work better for women. Thus, the real institutional change necessarily involves influence in political forums(Rao \& Kelleher, 2005). This should be enacted partly through the technical and political roles of feminist advocates. In practice, they are achieving institutional change through their technical roles by showing what the organization benefits from integrating gender issues and meeting official development priorities. Technically, they develop frameworks, checklists, and tools for gender incorporation in policies, programmes and gender-awareness raising and planning. However, their political roles, which, as stated by Standing (2004) are the appropriate space and place for driving transformation, are challenged in the way that current development industry privileges technical roles and rejects political roles(Mukhopadhyay, 2004). As a result, the political project of gender quality is completely overlooked because of the resistance to the project of equality between men and women that the language of politics is likely to bring when the word 'equality' is used. Hence, political projects have been replaced with study tours and other gender requirements in programme management (Standing, 2004). 
Standing (2004) added more support to why gender mainstreaming has not succeeded in influencing politics. Those working in gender and development are young and enthusiastic officers and attend post-graduate programmes or training. They are not experienced development professionals who can be influential in funding agencies. In the development industry, gender and development is professionalized mainly through providing academic courses. Trainers in these courses are academics often with feminist backgrounds. This professional training is undeniably an excellent point for theoretical analysis. However, social transformation in gender relations needs to have the involvement of academics, practitioners, aid workers and others who have a background in political activism.

Additionally, there is a certain naivety among these cadres about how real world institutions work, particularly in regard to the roles and functions of institutions which set policy or mediate its implementation. As such, the improvement of policies on gender need to be carried out with political alliances, not just through written statements or directives for the bureaucracy to follow (Standing, 2004). The gap between the intentions and the outcomes of policy enforcement also undermine gender mainstreaming. The complexity and sophistication of gender and development has not been taken into consideration in issuing related policies. In reality, the outcome of policy implementation is mostly determined by a mechanical belief in the power of intention while unintended consequences are not acknowledged (Standing, 2004).

Gender mainstreaming has become the dominant strategy for achieving gender equality in developing regions such as Africa, Southeast Asia and the Pacific. In many cases, agendasetting is often made by development practitioners and elite members of any given society. It is often accepted that Western-based gender mainstreaming experts continue to control agendas and resources, coming to impose a particular policy framework that may not be useful in a local context. In other words, gender consultants sell ideas which may or may not be appropriate or considered important and valuable in that local context. Thus, under many circumstances, gender may be a threatening import, which can result in resistance to the policy of gender mainstreaming. Or the implementation of gender mainstreaming policies is subverted to the other demands (Lyons, Curnow, \& Mather, 2004). 


\subsubsection{Insufficient attention to and/ or the resistance to gender mainstreaming among stakeholders}

Most organizations use a combined approach by which all staff share responsibilities for gender issues but are supported by gender specialists. Consequently, the success of gender mainstreaming very much rests on key individuals in an organization. Gender issues can therefore disappear when responsibilities for mainstreaming are taken by all staff at the same time. Tiessen (2007) cautions that when gender mainstreaming is thought to happen everywhere within the organization - because it is assumed to be taken into considerationin all areas of an organization's work, such as programming, project design, implementation, budgeting, and recruitment policies - then the mainstreaming happens nowhere because responsibility is too widely dispersed throughout the organization. Then the process is at risk of being decentralized to the point of being completely invisible. Or, as Lewis (2005, p. 125 cited in Tiessen, 2007, p. 18) stated, “Once you've mainstreamed gender, it's everybody's business and nobody's business".

In organizations like NGOs, the staff (especially senior management) view organizations as gender-neutral workplaces because gender inequality is somehow perceived to occur elsewhere in communities and the rural areas where they are working, rather than in the institutions themselves. Where this has been the case, there is a need for on-going training which must be relevant, consistent, specific and culturally-sensitive (A. Moser \& Moser, 2005). Promoting women's participation is an integral part of gender mainstreaming, yet mainstreaming is more than a question of boosting numbers of women in an organization. Specifically, all staff in an organization may be committed to full engagement, but in practice this is not always the case (Kabeer, 1994; A. Moser \& Moser, 2005; Plowman, 2003).

Mainstreaming was adopted in Beijing with the aim of bringing gender equality to centre stage. However, most development institutions have had to be reminded of the need for gender analysis in their work while policy-makers have had to be lobbied to include gender (Mukhopadhyay, 2004). At work, colleagues need to be persuaded of the qualitative difference that gender analysis could make were it to be integrated. Or else, gender is viewed neutrally in development planning. Here, it is necessary to flash back to the early intention of gender mainstreaming which promised to make gender-based biases and 
injustices known to all systems, structures, and institutionalized cultures with a view to removing them. The Beijing Platform for Action came along with many measures including the establishment of national machineries, so that gender mainstreaming was inclusively ensured in all areas of society and responsibilities of all people. However, it was interpreted as solely duties of some gender focal point staff, units or agencies (Mukhopadhyay, 2004; Woodford-Berger, 2004).

The picture of gender mainstreaming is greyer and often seen as positioned between 'a rock and hard place'(Rao \& Kelleher, 2005, p. 59), thus meeting resistance at different levels. At the macro level, there is increasingly hostility towards justice and equity in policy environments. At a meso level, gender mainstreaming in organizations is included in strategies and activities. But its practice does not always transpire as hoped. Moreover, resistance to the value of women's rights and gender equality goals means that priorities, poor infrastructure, and funding cuts often push gender concerns further away from the centre. At a micro level, MDGs do have measurable indicators for women's empowerment, but limit the possibility for change by leaving violence against women unattended to (Rao \& Kelleher, 2005).

The process of gender mainstreaming becomes more challenging if an NGO is male-biased in its organizational culture in regards to attitudes, hiring, working conditions, structures and procedures (A. Moser \& Moser, 2005; Plowman, 2003). Consequently, resistance (mainly male) to gender issues, which may be from management, staff and field workers is sometimes felt. Resistance and negative attitudes toward gender issues are frequently attributable to the lack of staff understanding of basic concepts or the relevance to their work and how to incorporate them into daily tasks. In development organizations, like in other workplaces, although there are more female employees, a masculine culture remains pervasive in work practices and promotional opportunities (Rea, 2004).

According to Rea (2004), women working in the development sector are mostly single because being married and having children can still be problematic to career paths in this field. This fact was found in the Australian workforce. In this sector, it is more likely for men to seek a better balance between family and work. The attitudes and cultures in development organizations are the major impediment to successful mainstreaming. The 
traditional masculine culture continues to be transferred in practice at home and in the field. There is a fear that the transformative potential of thoroughly addressing gender discrimination and oppression may disrupt both domestic and professional spheres. Therefore, 'cleaning up one's own backyard' should be regarded as a top priority, as recommended byRea (2004, p. 44).

\subsubsection{Lack of resources for gender mainstreaming efforts}

The implementation of gender mainstreaming has been inconsistent and involved only a few activities including data collection, short training courses, organizational policies and gender focal point staff, rather than a coherent and integrated process (A. Moser \& Moser, 2005; Tiessen, 2007). The main cause for the lack of change is policy evaporation: policy commitments to gender mainstreaming often fade out in the planning and implementation stages. In addition, there is an on-going lack of staff capacity, financial resources and appropriate organizational cultures (DAC, 1998; Derbyshire, 2002; Valk, 2000 cited in C. Moser, 2005).

The lack of staff capacity to implement gender mainstreaming strategies or carry out gender analysis is especially highlighted in the UN for example (Mitchell, 2004). Even the UNDP's committed leadership, policies, checklists, model gender-sensitive instruments and instructions, and staff gender performance appraisals cannot ensure successful gender mainstreaming in its activities and operations because its staff do not have the very complex skills to help them understand deeply the lives of the oppressed and the factors that shape and distort them(Reid, 2004). These skills allow people complex insights into the reality of the life situations of the powerless (Reid, 2004). From this point of view, it is necessary for people to not only well-conceive and implement gender mainstreaming strategies but also better understand the conditions for transformation in any particular setting through developing these capacities in an organization(Reid, 2004).

Moreover, gender equality is not institutionalized into the daily work of State and development agencies. The lack of financial resources for implementation is often cited as another constraint to moving the gender agenda forward (Mitchell, 2004). Furthermore, in an organization's thinking and practice, the focus is placed on efficiency and results, rather 
than dealing with power dynamics and cultural change. Organizational structures have a tendency to consolidate the power of a few, who are often unwilling to lose their power and privileges. Decision-makers are often uninterested in gender equality (Rao \& Kelleher, 2005).

\subsubsection{The lack of accountability mechanism}

Finally, the enormous failure of gender mainstreaming in institutions is due to the absence of professional and political accountability and the lack of institutional spaces through which an accountability system can be enforced (Mukhopadhyay, 2004; Rao \& Kelleher, 2005). Accountability structures cover performance appraisal and monitoring through to measurement systems. These are problematic in a sense because they cannot capture the full range of gender equality outcomes, both tangible and intangible. For the most part, gender mainstreaming relies on the commitment and goodwill of gender focal point staff. In some other cases, gender mainstreaming means nobody takes charge of its implementation.

Secured accountability for ensuring that gender equality is integrated into the policy-making process and programme implementation on a sustained basis is hardly present. It is a great challenge to hold those concerned accountable for gender work implementation (C. Moser, 2005; Mukhopadhyay, 2004; Rao \& Kelleher, 2005; Woodford-Berger, 2004). Programmes and/ or projects are largely negotiated, designed, managed and monitored by both international donors and local counterparts along with the possible participation of politicians and civil society. Initial consultations with stakeholders may be carried out, again as a condition of aid. Yet they never can go beyond this to develop sustainable ways to hold programme implementers accountable for outcomes (Standing, 2004).

\subsection{Conclusion}

There have been different feminist approaches to addressing gender inequality over time. They include Women in Development, Women and Development, Gender and Development, and gender mainstreaming recently. Gender mainstreaming was launched in Beijing in 1995. It was widely adopted by governments and development agencies right away and throughout the world with the common expectation that they could solve persistent and pervasive gender issues. By definition, gender mainstreaming seeks to put gender at the 
centre of strategies, policies and programmes by getting both men and women involved in the whole process so that they can have equal benefit, opportunities and access in all areas. The practice of gender mainstreaming, however, far from reaches those goals. A decade after introducing the concept, its progress was thoroughly and widely evaluated with a view to measuring its effectiveness as a tool for addressing gender concerns. Sadly, the findings showed more failure than success in implementing the strategy. A number of development practitioners, academics and feminists around the world provided examples of and reasons for this failure.

They all identified common main causes including: (i) lack of conceptual clarity; (ii) lack of political will and institutional change; (iii) lack of resources dedicated to undertaking this process; (iv) existing resistance to the notion of gender equality; (v) the absence of an appropriate accountability mechanism; and (vi) lack of staff capacity. So, the question arises should gender mainstreaming remain? Recently, the concept and framework of intersectionality has been discussed as an alternative to gender mainstreaming. It seeks to understand the differences among and between women and men and the ways that these interact to exacerbate marginalization(Thomas, 2004). In addition, it attempts to consider the diverse situations of women in the real world, taking into account other issues such as class, race, ethnicity, religion, geography, migration, mobility or immobility, and sexual orientation, causing simultaneous multiple oppressions (Thomas, 2004). All these situations inspired my research and its look at current trends and situations of efforts to implement gender mainstreaming in the context of development NGOs in Vietnam. 


\section{Chapter 3: Methodology}

\subsection{Introduction}

This chapter explores the methodology and methods employed to investigate the four research questions articulated in Chapter 1. It is divided into three main sections. The first section outlines the research methodology and methods. The second section describes the coding and data analysis process. The last section presents an overview of my positionality and reflections on my field research.

\subsection{Feminist Methodology and Qualitative methods}

Harding (1987) has written clearly on feminist research including epistemology, methodology, and method. An epistemology is understood as "a theory of knowledge" (Harding, 1987, p. 3). Epistemology is a philosophical theory that indicates a set of assumptions about the social world - "a fundamental belief system about who can be a knower and what can be known" (p.3). These presumptions are provided such that a researcher may make decisions on what is researchable and how to do that research. A methodology is a "theory of how research does or should proceed" (p.3). A method is a "technique for (or way of proceeding in) gathering evidence" (p.2). In a general sense, a feminist epistemology takes gender as central to understanding the production of knowledge and this perspective influences the nature of research performed and interpreted from this methodology.

To examine the extent to which NGOs based in Vietnam mainstream gender in their development work, a qualitative methodological approach was used. The qualitative approach helped me to investigate and understand the actual situation of gender mainstreaming in the case study organizations. Qualitative research tends to be an involved and flexible paradigm, which generates answers to questions by examining social settings and the individuals living in these settings (Bryman, 2008). Qualitative research offers information, local knowledge, expertise and experiences directly shared between researcher and participants (Mikkelsen, 2005). Furthermore, it can enable the researcher to deal with many factors such as empirical materials, personal experiences, interviews, artefacts, 
cultural texts, observations, historical, international and visual texts that illustrate routine and problematical moments and meanings in individuals' lives (Denzin \& Lincoln, 2005).

Within this qualitative approach, I have chosen to work through case study organizations. A case study is a system which is situated within the boundaries and within significant features such as context. Its activities are patterned within this bounded system(Stake, 2005). The case study research as the exploratory, descriptive and explanatory research focuses on individuals' experiences of some phenomena and views with an attempt to uncover particular complex factors underlying each case as a whole unit (Stake, 1995; Yin, 1994 cited in Denscombe, 2007). Using the case study allows a researcher to learn enough of an instance, or a few instances, to encapsulate the complexities of a given setting and see how various parts of that setting affect one another (Stake, 1997 cited in Johnson \& Christensen, 2008).

In summary, there are strengths and advantages to a case study approach as follows (Denscombe, 2007; Johnson \& Christensen, 2008). Firstly, it allows the researcher to use a range of sources, types of data, and research methods as part of the investigation in order to understand the complex reality under scrutiny. Using multiple sources of data can facilitate the validation of data through data triangulation. Secondly, this approach is concerned with investigating phenomena as they naturally occur, so the researcher does not have to put his or her controls over circumstances. Lastly, the case study approach can be suitable for smallscale research because it concentrates on one research case (or just a few cases).

However, one major weakness of case study research lies in the difficulty of generalization when the range of the study is limited to just one or a few instances of a broader class of things (Denscombe, 2010). The concern confronting anyone who adopts this approach can be addressed by considering the level of similarity between the case example and others of its type, and the extent to which findings from the case study can be applied to other examples (Ragin \& Becker, 1992; Yin, 2009 cited in Denscombe, 2010).I have employed it here to describe, discuss, and explain the gender concerns addressed in the development work of five organizations involved. As Denzin and Lincoln (2005) note, the use of case studies allows further investigations into the research question and generalizations to be 
drawn from specific local contexts; the results produced by my case studies are relevant to the contemporary practice of gender mainstreaming in NGOs in Vietnam.

To generate data, I used three methods. Firstly, secondary data was collected from the network of local organizations working on gender - Gencomnet, INGO centres - and websites of the Vietnam Women's Union, the National Committee for the Advancement of Women (NCFAW) and UN Women in Vietnam. Casual conversations with those who were working in the development field, and on gender in particular, also brought about some useful contextual data for the research, and has been classified as secondary data. In addition, a desk review was carried out to understand NGOs' activities in Vietnam, gender issues in Vietnam, the current institutionalization of gender mainstreaming in organizations and their development work and organizational studies. Lastly, primary data was gathered through semi-structured interviews with voluntary informants from five organizations including three INGOs and two LNGOs. Of these, one INGO and one LNGO became the main cases for analysis while three others provided supplementary information to consolidate and better generalize the findings.

\subsection{Data collection}

\subsubsection{Secondary data}

As one of qualitative methods, secondary data analysis was used in this research to bring about a broader understanding of real local contexts and practices. This data was gathered from various sources and organizational studies.

\section{(1) Data sources}

My understanding of the local context was widened thanks to data sourced from LNGOs, development organizations in Vietnam, and the Vietnamese Governmental agencies. The legal documents and national regulations regarding gender issues in Vietnam were available at related governmental bodies' websites such as Vietnamese Women's Union (www.hoilhpn.org.vn), and National Committee for the Advancement of Women (www.ubphunu-ncfaw.gov.vn). The websites of a number of local and international NGOs' networks such as Gencomnet (www.gencomnet.org), and INGO centre 
(www.ngocentre.org.vn) were also used. As well, some relevant documents were gathered from various donor websites including the World Bank (http://www.worldbank.org/vietnam), the Asian Development Bank (www.adb.org/vietnam) and other related international development organizations based in Vietnam such as UNDP (www.undp.org.vn) and UN Women (formerly UNIFEM) (www.unwomen.org). I reviewed the literature at both national and international levels to construct a global picture of gender mainstreaming and to enable me to make comparisons between local and global contexts and organizations. The literature covered academic studies on international commitments and their impact on gender equality, NGOs' gender strategies, policies, audits, and gender mainstreaming guidelines to see how gender issues have been placed in the mainstream and addressed in reality. This review helped to understand the different lessons and approaches of those concerned in mainstreaming gender in their development work and how they contribute to achieving the ultimate goal of gender equality.

\section{(2) Organizational analysis}

A deep understanding of gender mainstreaming in NGOs was attained through analysis of organizational studies conducted in a number of INGOs and LNGOs. The organizational studies consisted of a critical approach to NGOs' project documents, project reports, organizational mandates, gender policies, strategies, planning, gender training documents, human resource management, and evaluation reports. The existing information available on these NGOs' websites, inputs from face-to-face interviews, and other related materials were gathered at the time of field research.

\subsubsection{Primary data}

\section{(1) Choice of informants}

The total number of voluntary informants participating in this research was 15 staff, from different levels of five organizations. Initially, I planned to involve four organizations as case studies in this study. I first set three criteria for selection of organizations including (1) development organization; (2) working for poverty reduction; and (3) with office based in Hanoi city. Based on those conditions, I contacted personal connections through whom I hoped to connect with some organizations while I was in Wellington, New Zealand. As time 
went by, it turned out to be very difficult to receive acceptances by distance, though I informed them briefly about the research aims, objectives, and processes of my study and kept track of their feedback. Some promised to consider but often responses were lost in the mail for quite substantial periods while others frankly refused. Therefore, I decided to wait to make formal contact until I got home. Upon my arrival, with the criteria I set earlier, I made a list of INGOs and their contact details from the directory on the INGO centre website (http://www.ngocentre.org.vn/). I began to contact these through emails and phone contacts. Over one week later, three contact persons on behalf of three INGOs agreed to take part in my research. Later, one of the three contact persons connected me with his colleagues for interviews while another asked me to contact colleagues of hers directly. The last organization admitted to having nothing to do with gender and development but was willing to talk to me if I wished.

My final decision, made in consultation with my supervisor, was to target the first organization as a main case, using the other two to provide supporting data. Finally, I got to carry out in-depth interviews with five people in the core organization, four in the second, and just one in the third. In the first case, I still missed interviewing one key informant. That person promised to meet me while Iwas in the field, and then cancelled due to her tight working schedule. Despite efforts to find another time, it was not possible to interview her. I then approached her boss instead and while he initially agreed to answer questions by email, to date he has not responded to any of my messages.

In the case of LNGOs, I also started the process of selection by making a list of potential organizations and contacting all of them. Unfortunately, all refused to be involved in my research for various reasons. Some said their organizations were understaffed or undergoing organizational restructures so no one was available for interview. Others said they were too busy to fit me into their work schedules. Some organizations expressed no interest in my research topic in the most frank manner by pointing out that there was nothing relating to gender in their work. It was at this point one of my friends managed to connect me to an organization thanks to her intimate relationship with its Director. In this organization, I became acquainted with a young girl who was working for a gender network of LNGOs (called Gencomnet). She proved to be very helpful and connected me with different 
organizations. Having two organizations willing and able to participate in my research, I stopped looking for additional organizations and started to make appointments for interviews.

The process of choosing case study organizations taught me some valuable lessons. The first was the importance of acquaintances and connections with people working in development. Securing a local organization's commitment to the study was a great challenge. I could not secure involvement without the personal relationship I had with my friends and their networks. Secondly, INGOs seemed to be more open to cooperating in research than their local partners. I felt quite comfortable explaining my research to those working for international organizations and received feedback from them quickly. Meanwhile, local organizations hesitated to take part in this kind of research for the reason that "sensitive" information held by their organizations might be made public by the researcher. Finally, those working in development organizations are very busy and I was not able to select interview subjects. Thus, I worked with whoever was available and willing to be involved in the research. Luckily, the key informants nominated by the organizations provided me with relevant information used for the study (see Table 3.1 for list of interviewees in two main case organizations).

\begin{tabular}{|c|l|l|l|}
\hline No. & \multicolumn{1}{|c|}{ Code Number } & \multicolumn{1}{|c|}{ Position } & \multicolumn{1}{|c|}{ Location } \\
\hline 1 & INGO 1.0 & Country Director & Hanoi \\
\hline 2 & INGO 1.1 & Gender coordinator & Hochiminh \\
\hline 3 & INGO 1.2 & HR manager & Hanoi \\
\hline 4 & INGO 1.3 & Project Officer & Hanoi \\
\hline 5 & INGO 1.4 & Project Manager & Hanoi \\
\hline 6 & INGO 1.5 & Program Manager & Hanoi \\
\hline 7 & LNGO 1.1 & Deputy Director & Hanoi \\
\hline 8 & LNGO 1.2 & Project Officer & Hanoi \\
\hline
\end{tabular}

\section{Table 3.1. List of Informants}

For INGO 1, I first contacted the gender coordinator who expressed his interest in my research. More than that, he helped to pick up other informants for my research based on 
my request for a number of staff at each level. My task was to follow-up and set up meetings with those people at suitable times. My process of choosing participants was very much facilitated through his arrangement. With INGO 2, I also talked to gender focal point staff first who recommended me to people as I needed. I then obtained their contact details through the organization's receptionist and approached them to request their participation in the study. For LNGOs, I worked through personal relationships to make connections and set up interviews with relevant available staff.

Although my research was carried out in five organizations in total, I have decided to focus on two organizations (one INGO and one LNGO); a necessary level of staff interest was not forthcoming in the remaining organizations. Nonetheless, information shared by their staff remained useful to the study, contributing to wider data and information. As a result, I was able to draw a more complete and transferable picture of current gender mainstreaming practice in NGOs based in Vietnam.

\section{(2) Semi-structured interviews}

Interviews were conducted using semi-structured questions to collect primary in-depth data for the study. Semi-structured interviews were used so I could interview with an open framework, allowing for focused, conversational, two-way communication. In this sense, the interviewer and interviewees were able to give and receive information comfortably. It also helped me direct the issues to be addressed and the questions to be answered while the flexibility of the method allowed me to establish new main and sub-questions if I found a given idea or issue interesting during the interview (Denscombe, 2007). Furthermore, it is much easier to transcribe a recorded interview when the talk involves just one interviewee. Significantly, this method can generate qualitative data about the interviewee's thoughts, beliefs, knowledge, reasoning, motivation, and feelings about a topic (Johnson \& Christensen, 2008). In this study, semi-structured interviews brought explicit data and information as to how and to what extent gender is mainstreamed in NGOs' work, factors undermining the translation of theory into practice, and challenges facing organizations in their efforts. It also informed the different approaches adopted by INGOs and LNGOs in integrating gender concerns in their activities. 
Prior to interviews, the key informants learned the topic of the interview, the purpose, and the process of the research through written and verbal information both in English and Vietnamese. Vietnamese versions of all research documents were made available, including the information sheet, the consent form, the proposal, the research aim, objectives and research questions, and the interview questions. I developed a general interview guideline outlining the main topic and questions that steered each interview. However, interview questions were modified according to the type of organization and the position of the informant. The guideline also had to be revised to reflect emerging themes and categories as the interviews progressed. The interview questions consisted of introduction questions, follow-up and probing questions, specifying questions, direct and indirect questions, structuring questions, silence, and interpreting questions (Bryman, 2008).

Twelve interviews were conducted and audio recordings were transcribed. One meeting was not recorded because it was conceived of as a preliminary discussion and was supposed to be followed by an official interview. At the end, it turned out that this informant was too busy to meet again. Another informant agreed to take this person's place and respond to emailed questions but thus far this person he has not responded. The majority of the interviews were carried out in informants' offices. One was held in a coffee shop. I conducted the interviews with local interviewees in Vietnamese and those with foreigners in English. The length of the interviews varied. Some lasted more than one hour and some less than thirty minutes, with an average length being about forty-five minutes. The difference in interview length was because the informants had different knowledge, experiences and backgrounds with regard to gender issues and mainstreaming in the organization. The length of the interview also depended on how much time the interviewees could give. During the interview, I took notes of the main points. I managed to write personal logs during the field period and after each meeting, I then transcribed the twelve recordings and summarised my notes from the unrecorded interview.

\section{(3) Participant observation}

Participant observation was one of the three data collection techniques I had mentioned in my initial research design. However, it proved unsuitable when in the field. The INGO agreed to take part in the study but refused to let me be based in their offices on a regular basis 
because they did not have space for me and were not comfortable with my presence. In addition, they indicated there would not be many activities for me to observe there because development workers were required to travel a lot to communities or project sites for gender work. Initially I was invited to attend one gender-training seminar organized by a network of NGOs and to witness the gender auditing of some project sites of an INGO. However, unfortunately these two events were delayed till August and September respectively, after I had returned to Wellington. Nevertheless, I often observed people's actions, behaviours and attitudes in daily life from gender perspectives, which contributed to bolster my understanding of gender issues.

\subsection{Limitation of the study}

I am an ordinary person, and have not been working in the development field for very long. I have been away from Vietnam, in New Zealand for over a year, studying for a Masters in Development Studies at Victoria University of Wellington. Therefore, my relationships with people working in the same field at home seemed to be limited. There are some other limitations of this study. Firstly, I could not reach and did not interview as many people in different positions at different levels in the organization as expected. Many did not respond to my requests for participation through email or telephone. The information from 14 interviews with people of five organizations represented here tells just a part of the story on how far gender has been mainstreamed in NGOs' development and daily works.

Those interviewed are at work in various positions in their organizations with different duties and responsibilities. Their involvement in gender work in some cases proved to be dissimilar and at different levels. They understood and perceived gender issues and mainstreaming differently. Moreover, these professionals were extremely busy. Those working for LNGOs, in particular the leaders were very bureaucratic. It was not easy to get appointments with some because their travelling schedules were very tight. Due to the time and cost constraints, I limited the scope of my study to those based in Hanoi city. This could be seen as a limitation to the generalization of the research results. Therefore, there might be relevant information not covered by the scope of this study. Nonetheless, my interview questions and other methods aimed at obtaining as broad an understanding as possible within these limitations. 
Originally when I designed the research, I assumed an ability to access organizations' documents such as mandates and policies prior to interview. These would enable me to adjust lists of questions relevant to the organizational information. Accordingly, what I obtained from interviews might be deeper and closer to the aims and expected outcomes of the research. However, often the relevant staff were busy and thus unavailable. In other cases, interviewees preferred to hand documents in after the interview. Furthermore, access to some of the latest figures and statistics from Wellington was difficult when subjects had hardly responded to my mail.

The majority of interviews were conducted in Vietnamese while one was done in English. Later all interviews were transcribed. As a non-native English speaker deciphering the meanings of words and phrases delivered in English interviews was challenging yet necessary, if my transcript was to be correct and coherent. Further, some Vietnamese words do not have direct translations into English. Some interviewers often quoted Vietnamese idioms and proverbs that were very hard to translate.

\subsection{Ethics}

My ethical application was reviewed and approved by the Human Ethics Committee at Victoria University of Wellington by 13 April 2011. Fortunately, I was able then to leave and start my field research in early May. In the field, the consent form was sent to all informants through emails for their review prior to the interview. Some requested slight changes to the form to emphasize the need for permission before publishing any product from the tape recording. Once the change was made, the form was signed. However, almost all interviewees gave me verbal consent instead. Nevertheless, I explained to all interviewees that the consent form was to protect them from unexpected issues arising at the completion of this study. Confidentiality was ensured to avoid any potential harm to the informants' positions in their organizations.

At the coding and data analysis stage, the interviews were quoted using pseudonyms. I used the term "INGO 1", "INGO 2", and "INGO 3" for international non-governmental organization numbers 1,2, and 3 respectively. Similarly, the term "LNGO 1" and "LNGO 2" signify local non-governmental organizations 12 respectively. I also used the coding number INGO 1.1 to 
identify individuals in order to protect them from any potential harm. Upon the completion of the thesis, all notes, tapes, consent forms and other related papers were filed in a safe place. Hence, no informants were identified by name or position or organization. A summary of research findings will be made available to those interested upon their request after the thesis has been examined.

\subsection{Coding and data analysis approach}

Coding is very important and the first step in data analysis is to search for meanings within the data (Hesse-Biber, 2010; Tolich \& Davidson, 1999). Coding is the process of grouping "the flow of raw reality into packages of items" and building the links between them (Charmaz, 1983 cited in Tolich \& Davidson, 1999, p. 142). In the coding process, quotes or expressions that sounded important based on the key research questions I already set from the outset were identified and then arranged into piles of groups (Lincoln \& Guba, 1985 cited in Bernard \& W.Ryan, 2010). Codes were used to give identify segments of text as a way of labelling, separating, and organizing data (Hesse-Biber, 2010). All transcripts were read and followed two procedures of coding to categorise and sort data: open coding (also known as literal coding or initial coding) (Strauss, 1997 cited in Tolich \& Davidson, 1999) and focused or selective coding. For initial codes, I used the informants' own words as quotes through which I tried to maintain some of the context for their utterances (Bernard \& W.Ryan, 2010). My literal codes did not have any disciplined form and reflected the areas of interest that I learnt from literature review (Tolich \& Davidson, 1999).

After this, I moved to a more focused coding procedure in which I no longer used labels to simply describe. Rather, I sought to make sense of codes that enabled me to develop an understanding or interpretation of what my informants conveyed (Hesse-Biber, 2010). In this phase, the focused codes were informed by the literal codes and with an analytical view (Bryman \& Burgess, 1994 cited in Tolich \& Davidson, 1999). As a result of this procedure, the literal codes were sorted into more abstract categories and so was my analysis level (HesseBiber, 2010). Through the whole coding process, I was able to re-affirm existing themes taken from the literature review, or create new themes emerging from the coding. Or, I applied a combination of inductive and deductive approaches (Bernard \& W.Ryan, 2010). Then I was able to make separate tables with themes, sub-themes and the transcript quotes 
cut and pasted in thematic files. The analytic approach I then used was thematic analysis as suggested by Grbich (2007) and Braun \& Clarke (2006 cited in Silverman, 2011, p. 275)which consists of five steps as follows:

1) Becoming familiar with the dataset (noting initial comments and ideas)

2) Generating initial codes (systematically coding whole dataset)

3) Searching for themes (collating similar codes into potential themes, gathering all data for potential themes)

4) Reviewing themes (checking if themes work in relation to the dataset, checking for examples that do not fit, generating a thematic map/diagram)

5) Refining themes (refining specifics of each theme and linkages between them, generating propositions, looking for complexity, associations)

In the interpretation stage, I re-examined the case studies based on the primary data collected from the field in combination with the literature review while being open about my personal involvement and experience (Neuman, 2006). I regularly checked the transcripts, the field notes and personal diaries over time to assess new and evolving ideas. Finally, I determined the key coding themes as follows:

\section{The case study of INGO:}

1.1. Locating entry points for GM process

\subsection{Approaches to $\mathrm{GM}$}

1.3. Challenges to the practice of GM

1.4. Potentials for INGO's greater contribution to gender equality in a long-term

\section{The case study of LNGO:}

2.1. Adapting GM approach based on the organizational context and characteristics

2.2. Domesticating key concepts to achieve implications of gender mainstreaming

2.3. Drawing attention to gender and organizational culture

2.4. Constraints facing the organization to make these ideas work 


\subsection{Positionality}

When conducting research, it is crucial to acknowledge the importance for a researcher to build up social and professional relationships with the researched in the field. Positionality is indeed a decisive role in defining the research process and its outcomes. In some ways, researching at home in my culture was one major advantage for me when communicating with the interviewees. As a result, I was free of worries to do with cross-cultural barriers. Another advantage was the open working environment I experienced where I was comfortable learning from and sharing with respondents. I built a few working relationships which provided me with assistance and facilitation. Undeniably, my career background, combined with my study abroad, drew respect and appreciation from interviewees. In particular some informants considered my topic relevant to the current vision of their organizations. The results of this study might establish substantial information, which could help them to improve their gender work.

By having the same appearance, speaking the same language, and understanding the cultural, political and social contexts, I was comfortable talking to the informants and able to build trust with them. Their understanding of the local contexts and working experiences made the interviews very interesting. In field research, I considered myself as an "insider" of local cultural norms. I am a woman representing those viewed as subordinate to men in my traditions. As such, there could have been a risk that I might speak to my natural oppositions and prejudices and thus impose my discomfort on male informants. Being aware of and feeling local women's sufferings caused by gender inequality could easily have stimulated me to try and make revolution whenever possible. However, I endeavoured to position myself neutrally with all interviewees in the most open and equal way. I could see that all male participants saw me very respectfully and equally as a result.

One of my male interviewees was very frank in telling me that $\mathrm{I}$, a woman with high education in her late twenties would have less chance of marrying a Vietnamese man. His explanation was that these men felt uncomfortable with wives who learned modern and open thinking and practised such attitudes towards every dimension in society, particularly gender roles. His frankness did not upset or discourage me, but served as a stimulus for my research. 


\subsection{Field research reflections}

To be a good researcher requires the organization to carry out interviews in terms of arranging location, managing time, dressing appropriately and designing useful questions. Travelling through Hanoi city, from accommodation to interview sites took approximately one hour by motorbike. I often had to carefully calculate time factoring in unexpected things like traffic jams in order to never be late for any interview. I spent time reviewing questions and sub-questions prior to each interview. At the same time, the aim and objectives of the research were also reviewed beforehand to make sure my questions were relevant and useful. The interviewees held different levels of responsibilities at their places of work. Some appeared very knowledgeable and aware of related policies, strategies, and guidelines. Some could gave only comments on issues within their round of work. I used different sets of questions for different interviewees based on their respective positions. Questions were also changed at times when my own observations raised emerging or interesting issues.

Sometimes, I felt informants misunderstood difficult questions about issues beyond their scope of work. For example, when interviewing informants about commitments to gender equality at international levels and national levels, variations of questioning and examples had to be provided before they understood the terms of my question. Sometimes interviewees did not understand concepts and terminologies. Above all, I always questioned all interviewees in a diplomatic, polite and respectful manner while maintaining an open atmosphere throughout the whole interviewing session. As a result of this, the interviewees were allowed to be honest and open.

Stopping the interviewee from talking about unnecessary and irrelevant information was challenging, especially in conversations with older people. In my culture, it is considered to be disrespectful if we, as young people, stop the older person's conversation. In the field, I interviewed one old informant in a high position in one organization, who kept going off topic and being longwinded. I had to use the most polite and tactful ways I could to take her back to focus on my questions. Nonetheless, my interference did not work perfectly as at the end, half of the information she provided was useless for my purposes. 
Health issues should also be noted among the very important factors affecting the quality of the interviews. For instance, one interviewee was in a breastfeeding period. She had a headache when she arrived for the interview. She did not concur with my suggestion that we postpone that meeting to another day. The interview proceeded as scheduled, but very uncomfortably. My questions had to be repeated until the interviewee understood the terms, and sometimes her answers were irrelevant and unfocussed. Personally, I occasionally lost motivation for the interviews because of some health issues. Travelling in the heat of summer over long distances was tiring, and my tiredness may have influenced the quality of the interviews to some extent. In another example, one informant did not feel well on the day of interviewing, and, during the interview, was anxious for it to end.

\subsection{Conclusion}

The whole research process used qualitative methods, including a desk review and the generation of secondary data and primary data through in-depth interviews. These data collection techniques are very commonly employed by researchers in the development field. This chapter pointed out some of the issues and complexities of the research, which might have had potential impacts on the research results. Positionality and reflexivity were also discussed in this chapter.

I was a local researcher and this helped me avoid many problems. However, some challenges still remained. Deciding how much work to carry out in the field was difficult. Stepping into the field to begin with was daunting. The situation in the field varied across types of organizations and individuals. Over time I learnt to stay flexible and focused in terms of research design, methodology, and methods with a view to dealing with every possibility and changes in the field. Despite some challenges in the field research, the interviews with informants ended satisfactorily. By the time I left the field, I had received sufficient information from my sources and informants to complete the thesis. The research findings were reinforced and enhanced by my understanding and perception of my home country's tradition, culture and contexts. 


\section{Chapter 4: Background and context}

\subsection{Gender role ideology defined by Confucianism}

Strong Confucian patriarchal values deeply embedded in people's views on women and men have persisted in Vietnam. The culture and tradition of Vietnamese families are greatly influenced by Confucianism as a result of the long period of Chinese colonization from 111BC until the mid-tenth century (Long et al., 2000; Dalton et al., 2001, p. 7 cited in Hoang, 2005). Confucian ideology determines patriarchal authority in the family as the norm. The responsibility for maintaining family happiness is vested in mothers and wives. Accordingly, their roles are domestically oriented, serving their husbands, bearing and rearing children, doing housework and looking after parents-in-law in their old age. They are thought to become men's dependants throughout their life (Pham, 1999; Tran, 1996 cited in D.Long, Ngoc Hung, Truitt, Phuong Mai, \& Nguyen Anh, 2000). Women are required to practise the so-called Confucian "four virtues" (Tứ Đức) of hard work, beauty, fidelity, and passivity and "three obediences" (Tam Tòng) to fathers, husbands and sons (Long et al., 2000, p. 32 cited in Hoang, 2005). The former means Vietnamese Confucian tradition holds that a woman belongs first to her father, then to her husband, and then upon her husband's death, to her oldest son.

The strong preference for sons in Vietnam is grounded in this patrilineal and patrilocal kinship system. A husband often insists on producing more children until a son is born. Sons are essential to their parents because they carry on family lines and names, perform ancestor worship, and take care of parents in their old age. People also prefer sons to daughters because they improve a woman's status in her family and confirm a man's reputation in the community, including his perceived masculinity. Men and women with no son often suffer from strong pressure within the husband's family, and experience humiliation within the community (Hoang, 2005).

Increasingly, many couples use ultrasound to know the sex of their foetus and may decide to abort unwanted female embryos. Male preference has resulted in an uneven ratio of boys and girls in Vietnam. The ratio of boys to girls in the age range from 0-4 has increased to 111/100 in 2011 from 108.65/100 in 2009 (Vietnam's General Office for Population Family 
Planning cited in Anh, 2011). Girls (rather than boys) are often required to drop out of school when families experience financial and economic shortages. Again, this stems from traditional thinking that education is less important for girls.

According to Hoang (2005), this appears to be more obvious and more serious in rural areas because men are often viewed as leaders and protectors of their wives and children. Men also tend to dominate conversations in public and at home. By and large, the World Bank in 1999 pointed out that the most common gender-related problems inside and outside the household in Vietnamese culture consist of an overburdened workload for women, maledominated decision-making, lack of women's voices being heard in agencies (the World Bank, 1999 cited in Hoang, 2005). Patriarchy underpins the functioning of the family. Wives should be compliant. They should not be seen as the main decision makers or income earners. Wives should be sexually available to their husbands and responsible for contraception. Husbands are regarded as having the right to discipline their wives and daughters.

The role of a wife is like a peacemaker and so domestic violence perpetrated by men rarely draws community criticism and is instead interpreted as the failure of the wife.

Reconciliation is preferably used to settle any domestic dispute. Consequently, separated and divorced women often carry the blame for their relationship's demise within the community. Unmarried women are perceived as an economic drain on their families. This creates pressure for young women to marry, and if unmarried they should remain virgins. The patriarchy of the household causes major cultural and social obstacles to efforts aimed at increasing the standing of women (ADB, 2002; Hoang, 2005; Knodel et al., 2004; Teerawichitchainan et al., 2008).

\subsection{Gender role in the context of economic reform}

The year 1986 was a milestone with the State and Government of Vietnam initiating economic reforms called DoiMoi. DoiMoi and the path to economic integration have achieved significant economic growth and poverty reduction. The reform lifted Vietnam's growth to be the highest in the world during the mid-1990s, at seven to eight percent a year. According to Inglehart and Barker (2000 cited in ADB, 2002), global economic development 
produces not only changes to some cultural values but also makes others more persistent. Vietnam's economic resurgence is no exception in this regard.

Economic reform was carried out to bring about economic growth and change traditional attitudes towards gender roles in society. Put another way, the reforms essentially challenged socialist policies, and aimed to combat centuries of Confucian influence, setting women free from the patriarchal system and their attendant domestic burdens. Yet the process of economic transformation and the privatization of public services have impacted on gender relations so that relations between men and women in households and society have become increasingly unequal both empirically and symbolically (Beresford, 1994 et al., cited in Knodel et al., 2004).

The market reforms initiated by the State came along with the reduction of state controls on individual and family life through the dismantling of collective structures and the subsidization system. Researchers argued that patriarchal traditions and values could return and potentially prove detrimental to Vietnamese women (Goodkind, 1995; Luong, 2003 cited in Knodel et al., 2004). In other words, the transition to a market economy could have undermined women's influence and underlined husbands' control in households (D.Long et al., 2000; Teerawichitchainan et al., 2008).

Some analysis has examined to what extent women's roles in families have been changed in response to structural and ideological shifts. The finding shows that retrenching in the state sector and removing the subsidization of daily care services has resulted in greater domestic burdens placed on women in the post-DoiMoi in Vietnam (Chen an Hiebert, 1994; ADB, 2002 cited in Knodel et al., 2004). In this sense, Vietnamese wives and mothers continue to take primary responsibilities for household chores such as food purchases, cooking, dish-washing, house-cleaning, and laundry. Women's work time is longer and they have less time for social activities. They are responsible for keeping families together through the unpaid tasks in married life although they are also being educated and being employed in non-farming sector. It is necessary to note that a minimal increase in husbands' involvement in these chores, specifically in the household budget management and pre-school child rearing, have been among the positive results of economic development (D.Long et al., 2000; Teerawichitchainan et al., 2008). 
In ADB's report (2002) indicates that, women prove to work longer than men in domestic sphere. This overloaded work badly influence women's health, particularly during pregnancy. Their domestic work constrains their access to education, social activities, and community decision-making process. As a result of improved living standard, many households in cities can afford to purchase kitchen's utensils and home appliances which somehow ease women's burden at home. However, the situation of women in rural areas is sadly worse in a way that men mainly make decision on the farming issues. Men in these areas - as family's breadwinners have more access to land, credit, and technical training on agricultural extension but women are not because their time is buried in housework (ADB, 2002; D.Long et al., 2000; Knodel et al., 2004; Teerawichitchainan et al., 2008).

Economic transition has led to the end of the cooperative agriculture system and greater productivity and poverty reduction, especially in rural areas. Regarding gender relations, women's positions have been challenged by changes in the economy. Those working for the State sector are required to retire five years earlier than men, limiting their opportunities for job promotion. Moreover, the privatization of the State sector cost a larger number of women than men their jobs. Such jobs were lost with neither compensation nor increased access to non-farm and private sectors. Women also tend to be employed in the informal sector' while men tend to be employed in the 'formal sector'. However, improvement for women has lagged behind that of men in certain areas and social groups. It is particularly more obvious to see pockets of gender disparity in poorer communities in which resources are scarce.

As economic reform has progressed in Vietnam, agriculture has gradually become less significant economically. The economic shift has made fundamental changes to the organization of agriculture production and gender relations within farming communities. Land reform has led to a fall in the numbers of male farmers by 0.3 percent while numbers of female farmers has risen by 0.9 percent annually. In this period, the new female entrants into the agriculture sector accounted for 92 percent as men moved towards non-farm occupations. Non-farm employment in rural and urban areas brings about new opportunities for both women and men. As the economic transition goes on, it is critical for both sexes to have equal participation in the economy; equal access to all forms of education, business 
skills and information technology; and equal access to productive resources, such as credit and political decision-making. Nonetheless, women from poor households or ethnic minorities are the most disadvantaged in Vietnam. Their situation has changed a little as a consequence of DoiMoi. Since the implementation of DoiMoi, Vietnam has achieved great progress in upgrading facilities and improving accessibility to community health care services with doctor, mid-wife or obstetrics-pediatric assistants in each health centre. However, some communities, mainly in poor and remote areas are encountering the lack of access to these services (ADB, 2002; D.Long et al., 2000; Knodel et al., 2004; Teerawichitchainan et al., 2008).

Realizing that fact, the Government of Vietnam is taking efforts to re-define gender relations in line with socialist ideology. In Vietnam in particular, the gender division of household labour over the last four decades appears to show a continuance of traditional family values, rather than considerable transformation of them. It is clear to see that very little has changed in gender beliefs and practices and in the empowerment of women. Social attitudes of inequality remain and are reflected both in public and private spheres (ADB, 2002; D.Long et al., 2000; Knodel et al., 2004; Teerawichitchainan et al., 2008).

\subsection{Institutional Frameworks for the Advancement of Women}

The Government of Vietnam has actively promoted the advancement of women through the establishment of national machinery for women, the promulgation of a number of policy frameworks, and commitments to international gender agendas. The national institutions for the advancement of women and gender equality in Vietnam include the National Committee for the Advancement of Women (NCFAW), Committees for the Advancement of women (CFAWs) and the Vietnamese Women's Union (the VWU) at all levels.

At the national level, some policy frameworks have been launched for the promotion of gender equality. They are the National Strategy for the Advancement of Women in Vietnam by 2010 and then 2020, the Plan of Action for the Advancement of Women in Vietnam by 2005 and the Comprehensive Poverty Reduction Strategy (CPRGS). To create legally favourable conditions for the implementation of these strategies, in 1986, the National Assembly ratified the Marriage and Family Law, which regulates equal responsibility of 
spouses for domestic chores and childcare (Tran, 1996 cited in Knodel et al., 2004). The Gender Equality Law was approved in 2006 and the Domestic Violence Law one year later (NCFAW, 2004a).

Significantly, Vietnam passed a regulation in 2010 , which entitled both husband and wife to the land use right certificate in agricultural land(MPI, 2008). At the international level, Vietnam was one of 180 signatories to the Millennium Development Goals (MDGs) in September 2000 and made strong commitments to CEDAW and the Beijing Platform of Action. It is worth noting that the Vietnam Development Goals (VDGs) were formulated on the basis of MDGs but set more specifically to the actual contexts of Vietnam in terms of gender equality and women's empowerment. Noticeably, these goals are integrated into the Socio-economic Development Plan 2006-2010 in Box 4.1(MPI, 2008).

\section{Vietnam Development Goals (SEDP 2006 to 2010):}

- Reduce gender disparity in primary and secondary education among ethnic minority groups by 2010

- Increase the representation of women in elected offices / positions at all levels

- Increase the participation of women in all sectors and agencies (including Government agencies, ministries, enterprises) by 3-5\% in the next 10 years

- Ensure that newly-issued land use certificates bear the names of both husband and wife

- Reduce the vulnerability of women to domestic violence.

Box 4.1: Vietnam Development Goals (SEDP 2006 to 2010)(MPI, 2008, p. 28)

National Strategy for Gender Equality 2011-2020 was approved in 2010 with the general aim of reaching real equality between men and women by 2020 in terms of opportunity, participation and benefit in the spheres of politics, the economy, culture, and society, and in 
contributing to the robust and sustainable development of the nation. This aim is central to the socio-economic development strategy of the nation and a ground for the human development strategy of Vietnamese State(Government, 2011). The strategy is divided into two phases -2011-2015 and 2015-2020 - and comprises seven specific objectives as follows:

Objective 1: Strengthen women's participation in positions of leaders in order to gradually close gender gaps in politics

Objective 2: Narrow gender gaps in areas of economics, labour, and workforce; enhance access of poor women in rural areas, ethnic minority women to economic resources and labour force

Objective 3: Improve quality of female human resource; gradually ensure equal participation of men and women in training and education

Objective 4: Ensure gender equality in access to and benefit from health care services

Objective 5: Ensure gender equality in spheres of culture and information

Objective 6: Ensure gender equality in family, gradually remove gender-based violence

Objective 7: Improve State's management capacity of gender equality

(Government, 2011)

The Gender Equality Law was proposed to the National Assembly by the VWU in 2005 with considerable opposition from a large number of senators who believed that gender equality was already accounted for in other laws. Eventually, this bill was passed to become law one year later. The Gender Equality Law aims to guarantee equal rights to women and calls for gender strategies at the ministerial level. The Ministry of Labour, Invalids and Social Affairs (MOLISA) was nominated to be in charge of enforcing this law. The Department of Gender Equality in this Ministry took responsibilities to develop a National Strategy for Gender Equality 2011-2020 and a National Target Programme for Gender Equality 2011-2015, which both contribute to implementing Gender Equality Law(VNGOs, 2006). 
The Domestic Violence Law was passed in 2007 and enacted by the Ministry of Culture, Sport and Tourism in the early stages of implementation. The Law regulates the prevention and control of domestic violence, protecting and assisting the victims of domestic violence. It outlines the responsibilities of individuals, families, organizations, and institutions in domestic violence prevention and control, and advises how to deal with breaches of the $\operatorname{Law}(N A, 2007)$.

At first sight, these laws appear to build a good legal framework to promote gender equality. However, the translation of these laws into real life is not going well due to problems in terms of institutional structures, accountability, leadership and knowledge, implementation, monitoring, evaluation, and reporting. More specifically, there is on-going confusion and overlap about roles and responsibilities between gender units. In some other cases, gender is an add-on responsibility to gender focal point staff in departments. In others, a clear strategy or guidelines on accountability are not made available. There is no system to retain those staff in charge of implementing, monitoring, evaluating and reporting on these two laws (VNGOs, 2006).

The confusion occurs in the following way. Prior to the issuance of the Gender Equality Law, the Vietnam National Committee was primarily responsible for gender equality. Since then, responsibility has been shifted to the Ministry of Labour, Invalids and Social Affairs, while the Ministry of Culture, Sport and Tourism is in charge of implementing the Law on Domestic Violence. However, there are some issues arising here: (1) there is no mechanism available to coordinate enforcement of these two laws; (2) the role was a completely new one for the Ministry of Culture, Sports and Tourism and hence they have not proved sufficiently experienced to enforce it effectively; (3) the Ministry of Labour, Invalids and Social Affairs faces issues with the inadequate capacity of its staff. Staff in the gender departments of these two ministries still mistake gender equality as specifically women's issues, and so any and all matters concerning women are directed to these departments(MDGIF, 2010).

Before the promulgation of these two laws, the National Committee for the Advancement of Women was in charge of addressing issues relating to gender equality and domestic violence. Due to the shift of this responsibility to the ministries, this Committee has no longer been actively involved. There is also an apparent gap between legislation and 
enforcement because of a lack of resources, skills, and time constraints. This situation is coincident with the re-emergence of patriarchal attitudes as the State withdraws from the micromanagement of social activities. The Government of Vietnam is committed to gender issues through a top-down approach to legislation and policy formation. The concern is that gender mainstreaming without rigorous policies and checks in place may undermine or reverse progress to date (MDGIF, 2010).

\section{Organizations working for the Advancement of Women:}

The State organizations working for women consist of the National Committee for the Advancement of Women (NCFAW) and the Vietnamese Women's Union (VWU).

NCFAW, an advisory body of the government, was established in 1993 and arose out of the Committee for the United Nation Decade on Women. NCFAW officially represented the government at the Beijing World Conference on Women in 1995. It has since developed into a network with different branches and levels separate from the government. NCFAW advocates for the government on the development and enforcement of strategy as well as the National Action Plan for the Advancement of Vietnamese Women to 2010. NCFAW also organized communication on CEDAW, the Strategy and National Action Plan, and set up education sessions for the National Assembly and People's Committees. It also provided for education sessions for staff of ministries and provinces to help enable them to mainstream the gender policy and monitor the strategy and the National Action Plan (ADB, 2002).

NCFAW has 16 members, from organizations such as the VWU, the Youth Union, the Peasant Union, and various government ministries. This committee is externally funded by UNDP, the Government of the Netherlands, and other bilateral donors, and assisted by foreign consultants. NCFAW is responsible for coordinating the country's response to the International Platform for Action for Women and the Beijing Declaration signed by Vietnamese Government in 1996 (ADB, 2002). Nevertheless, many shortcomings and disadvantages have limited the effectiveness of activities for the advancement of women (VNGOs, 2006). Specifically: 
- NCFAW has had trouble shifting ideas into the governmental structure because the leader of NCFAW is not a member of the government;

- NCFAW lacks enough experienced Vietnamese specialists;

- The budget for NCFAW is limited.

(VNGOs, 2006)

The VWU is a mass organization, established in 1930 with 13 million members at all levels: national, provincial, district, and commune. In the past, it was mandated to mobilize women politically in the fight for independence. Once Vietnam gained liberation, the VWU played a central role in advocating for women's rights and benefits, and consulting on issues relating to women and children as instructed in Government Decree No. 163 in 1988 to bring about gender equality. In the context of increased international intervention since DoiMoi, the VWU has shifted its role of political mobilization and advocacy to implementation of development projects, such as microcredit and microenterprises development schemes, mother and child healthcare and family planning, agriculture extension, and domestic violence (ADB, 2002; VWU, 2005).

The VWU has cooperated with international agencies to implement projects due to its reach at the grass-root level. However, the VWU was stretched when having to concern itself with all gender issues because of its existing levels of skill and capacity. Moreover, the VWU faces challenges in building a team of capable and knowledgeable staff who can work as consultants in monitoring the development and implementation of equality policies as well as in adjusting the VWU's activities. Thus, the VWU remains suitable and beneficial to women (ADB, 2002; VNGOs, 2006).

Considering gender issues as a top priority in its development agenda, the VWU has adopted Gender and Development as an approach and gender mainstreaming as a strategy to overcome the above constraints and achieve the goal of gender equality. In 2004, with assistance from external donors, the VWU developed Gender Mainstreaming Guidelines, which are very comprehensive, specific, and accessible to all stakeholders. Nonetheless, major current concerns such as the confusion of key concepts such as gender, gender 
equality, gender equity and women mainstreaming and gender mainstreaming, are potentially undermining the process of gender mainstreaming in Vietnam.

This confusion occurs in almost all related discussions and debates so those involved often mistake gender for women. A number of leaders, civil servants and even policy makers are still unfortunately unaware or unconvinced of the intimate link between gender equality, poverty reduction and sustainable development. Their lack of understanding constrains attempts to mainstream gender into the national development agenda. Furthermore, the importance of gender mainstreaming as the most effective and popular approach to gender equality has not been widely recognized. Thus, some at even the central level continue to see gender issues as solely to be addressed by NCFAW and the VWU, rather than as something for which responsibility should be shared by all levels and all sectors (NCFAW, 2004a).

\subsection{Progress vs Impediment in addressing Gender Issues in Vietnam}

Vietnamese women have been acknowledged as playing an important part in national development. Thus, President Ho called for Vietnamese people to let go of historical prejudices and injustices against women (Desai, 1998 cited in Knodel et al., 2004). Equal relations between men and women have long been regarded as crucial for socialist state development. In 1930, and as one of the ten main tasks of the Vietnamese Revolution, President Ho stated:

We must respect women. One of the aims of the revolution is to ensure equality of rights for the women. Lenin taught us: women make up half of society. Society cannot be totally free so long as the women are not freed. Women must fight for their right to be equal with men. Men must respect them. The Party cell must educate its members and the people in respecting equality between husband and wife. (the VWU, 1969 cited in Mitchell, 2000)

When the Communist Party of Indochina was founded (Fahey, 1998 cited in Teerawichitchainan et al., 2008), the Party aimed to replace traditional Confucian-based ideologies - seen as the root of inequality between women and men in Vietnam - by eradicating poverty and women's domestic roles. In two wars against French and American 
invaders, Vietnamese women took enormous responsibilities for maintaining agricultural and industrial production at home while men were in battle. More than that, a great deal of women jointly took part in the army with men. The 1930 s call for equality between men and women was institutionalized in the Constitution of the New Democratic and Republic State in 1946 (Teerawichitchainan et al., 2008).

The idea of equal relations between men and women in Vietnam society is widely recognized as one of the legacies of the socialist revolution. In the first decade of the $20^{\text {th }}$ century, Vietnam launched the struggle for women's rights (nữ quyền) by addressing issues of literacy, marriage conditions and participation in the public sphere. Accordingly, the liberation of women (giải phóng phụ nữ) is seen as a condition leading to national liberation. Despite being viewed as a symbol of the national liberation, however Vietnamese women and gender relations were somehow neglected in the focus on achieving national independence and unity (Marr, 1985; Enloe, 1990; Nguyen, 1995 cited in Knodel et al., 2004).

Gender issues returned to the agenda in the late 1980s when people in social science, development and policy-making fields started to use the term 'gender', rather than 'women' as a sociological category. This shift was made on the basis of recognition that development is not only for and about women, but also about the relations between men and women. This idea was supplemented by the realization of scholars and researchers that even genderneutral policies may cause crucial consequences to gender relations. By this time, campaigns were launched to make the goal of gender equality known nationwide, to educate people about equality in areas of marriage, the labour market, and sharing domestic work between men and women (Liu, 2002 cited in Knodel et al., 2004).

Vietnam has made progress in closing key gender gaps, most notably in education, employment, and in increasing women's representation in the National Assembly. The gender gaps in education have been closing as seen in the Table 4.1. 


\begin{tabular}{|l|l|l|l|l|l|l|}
\hline & & & & \\
\hline
\end{tabular}

\section{Table 4.1. Level of Schooling Completed by Age and Gender (percentage completed)}

While gender parity has been achieved at undergraduate level, only 30.5 percent of Master's degrees and 17.1 percent of PhDs were awarded to women in 2007 (MOLISA 2010). The National Strategy for the Advancement of Women by 2020 sets a target of female Masters Holders to reach 40 percent by 2015 and 50 percent by 2020 and female PhD holders to 20 percent in 2015 and 25 percent in 2020 . Women are likely to enrol in social sciences and humanities while men dominate in the engineering field (29 percent enter engineering compared to 11 percent for women). Another concern relates to ethnic differentials in educational achievement. Educational indicators show that some provinces, especially those in the Northern Midlands and Mountains region, show high discrepancies between men's and women's levels in literacy and educational attainment. 
Another big gender concern in Vietnam recently is gender based violence which is defined as the purposeful acts of certain family members that cause or may possibly cause physical, mental or economic injuries to other family members. Almost two-thirds of Vietnamese women believe it is acceptable for men to beat their wives(UN, 2010). The study in 2006 conducted in three provinces of Thai Binh, Lang Son, and TiengGiang in Vietnam by the VWU revealed that, 40 percent of women were hit by their husbands. According to the study, 66 percent of divorces were attributed to domestic violence (WB, 2006). The pattern of abuse in Vietnam includes physical and verbal abuse. There were 21 percent of women being verbally abused by their husbands. Still, women's highest chance of being the victims of violence was in their own houses and involved with male alcoholism and financial constraints (Kabeer et al.,2005 cited in WB, 2006)

The impact of violence is significant. It can lead to physical injury (in the cases of 26 percent of those physically or sexually abused) and victims are much more likely to report poor health and emotional distress. Over 30 percent of those suffering violence have had abortions, compared to just over 20 percent of those not experiencing violence. Women experiencing violence report more problems with carrying out daily activities, memory loss, and difficulties walking. Their children have a higher rate of behavioural problems ( 27 percent) than those of non-victim women (16 percent). Women experiencing violence report that they usually keep these events secret, even from close family members. When they do report incidents to the police or other authorities, complaints are usually dismissed and they are told that these are family matters. As discussed earlier, in relation to Confucian gender ideology, often women feel a responsibility for their abuse, because, as women, they feel their role is to create a good family life (ADB, 2002).

Regarding women's representation in politics, the National Strategy and Plan of Action for the Advancement of Women until 2020 and Vietnam Development Goals (VDGs) have set specific targets for women's participation in leadership and management. The Law on Gender Equality 2006 lays out concrete measures to move forward on gender equality in politics. Women in leadership at Party Committees at all levels should reach 25 percent and higher by 2020; female members of parliament and people's councils at all levels should be 35 percent and higher in the term 2011-16, and above 40 percent in the term 2016-20; and 
government bodies and organizations are to maintain more than 30 percent female leadership and are required to have women as key leaders in such organizations.

In Vietnam, women account for 49 percent of the total labour workforce. Gender equity in employment is measured through the proportion of women working in both the agricultural and the non-agricultural sector (MPI, 2008). However, there is gender disparity in some employment sectors as reflected in Table 4.2:

\begin{tabular}{|c|c|c|}
\hline & & Unit \% \\
\hline & Female & Male \\
\hline Total & 49.4 & 50.6 \\
\hline \multicolumn{3}{|l|}{ By gender } \\
\hline Agriculture, forestry and fishery & 51.6 & 48.4 \\
\hline Mineral exploitation & 31.1 & 68.9 \\
\hline Manufacturing and processing & 51.7 & 48.3 \\
\hline $\begin{array}{l}\text { Production and delivery of electricity, gas, steam and } \\
\text { warm water }\end{array}$ & 17.3 & 82.7 \\
\hline Hotel and restaurants & 71.6 & 28.4 \\
\hline Banking and finance & 52.4 & 47.6 \\
\hline Science and technology & 34.0 & 66.0 \\
\hline Education and training & 69.2 & 30.8 \\
\hline Health care and social activities & 59.5 & 40.5 \\
\hline Culture and sports & 48.8 & 51.2 \\
\hline Administration and support services & 50.8 & 49.2 \\
\hline
\end{tabular}

Table 4.2. The proportion of men and women aged over 15 holding a job in selected employment areas(GSO cited in MPI, 2008)

More females are employed in non-farm sectors, particularly requiring highly technical inputs, and in service and private sectors (MPI, 2008). However, a majority of women remain working in less-skilled employment areas and in low positions in technical jobs. Additionally, the salaries of women are only 87 percent of those of men working in the same job. In midlevel technical occupations, women's incomes are only 82.9 percent of men's. In the 
processing industry, females earn only 77 percent of their male counterparts' incomes. In low-level technical or vocational levels, women are paid only 78.5 percent of men's salaries despite holding the same qualifications (MOLISA cited in MPI, 2008). Table 4.3shows the involvement of women in different employment areas.

\begin{tabular}{|l|c|}
\hline \multicolumn{2}{|c|}{ Unit \% } \\
\hline & Women \\
\hline Proportion of female labour (whole country) & 49.40 \\
\hline Leadership/ Managerial positions & 20.22 \\
\hline Highly professional/ technical & 47.20 \\
\hline Middle professional/ technical & 58.44 \\
\hline Ordinary staff in other areas & 45.45 \\
\hline Personal services, security guards, technical sales & 59.30 \\
\hline Technically-related employment in agriculture, forestry and fisheries & 42.64 \\
\hline Technically - related craft workers & 35.98 \\
\hline Installation and operation & 14.76 \\
\hline Unskilled & 53.64 \\
\hline
\end{tabular}

Table 4.3. Proportion of females employed against total labour force of 2007(NCFAW, MOLISA cited in MPI, 2008)

There are many issues of concern. The percentage of female leaders and managers is low, especially given the increasing rate of female workers generally. In the government currently, counting just the ministerial positions and excluding the positions directly relating to state budget and finance, Vietnam ranks the $89^{\text {th }}$ in a list of 93 countries in terms of how highly women are represented in government. Vietnam has only one female minister out of a total of 24 ministers. Recently one woman was named to the Politburo of the Central Committee Communist Party. Representation is low among business leaders and in administrative agencies. The voice and representation of women from ethnic minorities also needs to be increased in both political processes and at the grass-root level(MDGIF, 2010; VNGOs, 2006). 
In Vietnam, a lot of research has aimed to gather insights into family planning programmes, contraceptive use, and reproductive health. Issues here concern both sexes. Family planning is targeted initially at women only, yet men make decisions about contraceptive use and the number of children in the family. Studies on sexuality and violence find that many women feel a lack of control in their relationships with men. Women's vulnerability may be increasing as some enterprises consider the costs around reproductive functions such as maternity leave and childcare responsibilities to be additional expenses (Croll, 1998; Trinh Thi, 1998 cited in Knodel et al., 2004).

Vietnam has gained achievements relating to gender in line with the MDGs and other measures. However, the issue of gender equality in Vietnam is not as simple as increasing the participation of women in political, social, and economic activities and services(Vijeyarasa, 2010). Vietnam is no exception to the fact that gender inequality is deep-rooted in society, and, left unattended, could further adversely affect national achievements. Vijeyarasa (2010) expresses concern that the number of women represented in governmental offices does not reflect equal power-sharing between men and women in decision-making processes. Despite there being more females in parliament, men outnumber women in administrative positions at the local level (MPI, 2008). Women's participation at all levels seems to be primarily as deputies. At the grass-roots level, political authority rests with men.

Since quantitative indicators of MDGs fail to reflect the situation of populations on the margins, as claimed by Vijeyarasa (2010), ethnic minority girls' enrolments in school are 12 percent lower than ethnic minority boys(the World Bank, 2006 cited in Vijeyarasa, 2010). Vietnam continues to fall short of gender equality promotion partially because of the government machinery responsible for promoting gender equality objectives. These agencies are criticized for their lack of "influence, resources and creativity" (the World Bank, 2006,p. 118 cited in Vijeyarasa, 2010, p. 94)and also the limited "mechanisms for coordinating policy with government units"(Goetz, 2003,p. 77 cited in Vijeyarasa, 2010, p. 94). 


\subsection{Development intervention for gender equality in Vietnam}

The government continues to promote gender equality by enacting the Law of Gender Equality. Over the last decade, international development interventions from NGOs, UN, and other donor agencies have addressed gender inequality and the promotion of women's empowerment. Thus, gender issues have been of concern in most development projects (NCFAW, 2004a). Most international donors in Vietnam are committed to supporting gender concerns and adhere to gender equity as a cross-cutting strategy. Many have shifted from a WID to a GAD approach. Operationally this has resulted in a shift of focus from women's projects to mainstreaming gender concerns into all projects (ADB, 2002).

Given that fact, the UN selected Vietnam to be one of eight pilot countries for its 'Delivering as One' initiative with an aim to enhancing the accountability of governments to women's rights. In joint efforts with government, the UN in Vietnam is committed to promoting gender equality and women's empowerment through the process of gender mainstreaming in their projects and programmes, advocacy, and monitoring and evaluation, and the establishment of good practices and specific targeted initiatives at their local offices. Despite strong commitments by the UN system and UN agency headquarters, the 2008 UNCT Vietnam Gender Audit identified key challenges to achieving gender equality and women's empowerment in the context of UN reform in Vietnam. Some shortcomings include a lack of awareness on and capacity for gender policy among staff, lack of accountability at management level to gender equality outcomes, lack of management support, and insufficient investment in staff capacity for gender among others. The Gender Mainstreaming Strategy was developed for the period from 2009 to 2011 as a response to the above-mentioned findings and to strengthen the UN's work on gender equality and women's empowerment in Vietnam (UN, 2009).

Other donors are keen to support gender-specific activities directly, but their interventions tend to be small and short-term. In order to improve the overall effectiveness of these interventions, it is critical to have coordination from NCFAW. Other donors working on gender-specific projects include the Asian Development Bank (ADB), Canadian International Development Agency (CIDA), Food and Agricultural Organization of the United Nations 
(FAO), Oxfam Great Britain (ADB, 2002). INGOs in Vietnam have increased in both number and funding since 1986. In 2006, this number was reported to rise from 210 in 1994 to 650. Accordingly, total aid rose to 217 million USD in 2006 from 40 million USD in 1993. Their projects are mainly implemented in poor and remote areas throughout the country. Their focus has been aligned with Vietnam's priorities of national socio-economic development, especially poverty reduction and sustainable development (MOFA, 2007). Vietnam has managed to create favourable conditions and a legal framework to maximize NGOs' resources to the national development process. NGOs in general have been very active in the area of GAD in Vietnam for many years. Their projects are usually small and targeted and often better suited than larger projects are for dealing with such sensitive areas as genderbased violence. Their activities are numerous, diverse, and widespread and hence NGOs are included in all gender-related recommendations to government.

After DoiMoi began in 1986, other Vietnamese NGOs were established and began to develop as form different from the mass organizations. Till 2006, there were more than 300 Vietnamese local NGOs working in different sectors (Source: Vietnam Union of Science and Technology Association), such as: poverty alleviation, enterprise development, infrastructure construction, education, health care, environment, HIV/AIDS prevention, legal support, gender equality, and women's rights protection among others(VNGOs, 2006). There are a number of local NGOs in Vietnam which, due to financial constraints, operate in partnership with INGOs. They function themselves as small groups of professionals and work for the government or donors, often calling themselves social development organizations(VNGOs, 2006).

Although Vietnamese local NGOs are relatively new, they have contributed to policy construction, development, and implementation where gender equality is one of the main purposes. Vietnamese NGOs, INGOs, Overseas Development Aid (ODA) from Sweden, USA, Poland, England and elsewhere, and international financial institutions such as the World Bank and the ADB have funded many projects to improve knowledge around gender equality for rural communities. Both the Vietnamese government and NGOs have made significant efforts to eradicate discrimination and to improve the position of rural women in an attempt to help them achieve equality with men both in the family and in larger society (ADB, 2002). 
The biggest challenge for the new Vietnamese NGOs is their legal status around their participation in government programmes and how they can serve as a resource. Currently, there is no system in place to draw on the voices of civil society and NGOs on issues relating to the enactment of Gender Equality Law and Anti-Domestic Violence Law. Hence, the State is being pushed to quickly promulgate the Law on Associations to enhance the formation of NGOs to join the government's efforts in solving social problems in general and gender issues in particular (ADB, 2002; VNGOs, 2006).

\subsection{Conclusion}

In sum, reviewing gender issues currently - and the mechanisms to address them- enables NGOs as pro-active stakeholders in the development sector to seize opportunities to make their contribution to bringing about more equal relationships between men and women in Vietnam. With understanding of the current situation and the political mechanisms for gender-related decisions, NGOs can find the appropriate way to fill the niche and address the issue effectively and comprehensively. From the governmental end, the review will serve as evidence of the value of the contribution of NGOs, as well as the institutional and operational challenges facing NGOs in their attempts to help the government achieve gender transformation in the long-term. 


\section{Chapter 5: Findings and Data Analysis in the INGO}

\subsection{Introduction}

This chapter addresses the research question through a case study of an INGO. It uses material generated from a desk review of gender strategies, frameworks, training documents, and semi-structured interviews with key staff in the organization. The participants are quoted using the following forms: (male) Gender Equity and Diversity and Learning Coordinator (INGO 1.1), (female) Human Resource Manager (INGO 1.2), (female) Project Officer (INGO 1.3), (female) Project Manager (INGO 1.4), (male) Programme Manager (INGO 1.5). This chapter portrays the extent to which gender mainstreaming has been implemented in the INGO and highlights current gaps between theory and practice in the specific context of Vietnam. It is divided into two main sections. The first section is a brief introduction of the organization. The second section reports on the findings from fieldwork. It is arranged with titles representing main-themes and sub-themes categorized through the process of coding and analysing data, discussed in Chapter 3.

\subsection{Overview of the INGO}

The NGO was a member of an International Lead Organization (ILO), which was mandated for "a world of hope, tolerance, and social justice, where poverty has been overcome and people live in dignity and security" (Doc 1, p.3). The INGO in Vietnam as a whole operated under the leadership and guidelines of both the ILO and the Regional Lead Organization. Since 1989, the INGO has worked in 22 out of 64 provinces throughout Vietnam to implement over 120 different projects. Projects focussed on emergency response and preparedness including avian influenza programmes, rural development and natural resource management - including water and sanitation - and health and social programmes. Despite this activity, a formal country agreement was not signed between the Vietnamese government and the ILO until 1991. Up to 2009, the INGO in Vietnam had more than 100 national staff, 15 international expatriates, and an annual programme funding of approximately 6 million USD. It has established partnerships with the People's Aid Coordinating Committee of Vietnam (PACCOM), People's Committees, and government agencies such as: the Department of Agriculture and Rural Development (DARD), the Centre 
for Rural Water Supply and Sanitation, the Department of Health, and the Department of Education, as well as mass-organizations such as the Women's Union, the Youth Union, the Farmers' Association, the Trade Union, and Local NGOs (Doc 1).

The INGO was subject to its International Gender Policy, which was issued in 2009. This Policy defined explicit commitments to support gender equality. The Policy reflected the lead organization's efforts to eradicate poverty and social injustice and sought to promote the gendered realization of dignity and human rights for girls, women, boys, and men. Like other members of the international system, the INGO in Vietnam was also required to apply a set of standards including the incorporation of gender and power-analysis into key organizational policy, planning and programming. The process of policy making, planning and programming was based on data disaggregated by sex, age and other relevant diversity factors such as ethnicity, religion, and caste. In these policies, plans and programmes, gender results must be clearly stated. To achieve gender results, the relevant and feasible gender-sensitive indicators had to be set for every stage of planning, implementation, monitoring and evaluation (Doc.2).

These standards were compulsory for all member organizations. At an organizational level, sufficient funding must have been ensured to meet gender requirements. The Policy also put the focus on addressing gender equality through human resource policies and practices. All country offices under the leadership of the ILO were required to track and report annually on gender balance in staffing and governance structures to implement specific strategies to balance male/female representation. The executive and senior management staff must have reported on progress on gender equality to beneficiaries, donors, and the public on a regular basis, and the organizational capacity for the implementation of the Policy must be evaluated and strengthened accordingly (Doc.2). 


\subsection{Findings and Data analysis}

\subsubsection{Locating entry points for Gender Mainstreaming (GM) process}

\section{a) Embracing the terminology}

Interviews were conducted with five people - two men and three women - in the organization who held different positions at different levels and had been working for a long time in the organization. Participants had been employed between five and 15 years. During interviews, some basic terms were captured relatively well by all informants. There was a consensus amongst these people that gender referred to both men and women and that it is different from sex. Gender equality referred to the recognition that men and women have different needs and priorities. In their words, gender equality also meant equal access and control to resources, equal opportunities for participation in and benefits from project or programme activities. For example, INGO 1.2 said, "gender is about men and women, not just women in isolation".

\section{b) Putting institutional mechanisms in place}

\section{* Gender Mainstreaming Strategy}

The standards and principles articulated by the ILO were good starting points for the INGO to consider gender issues in its development work in the specific context of Vietnam. The year 2008 became a milestone for the official emergence of gender work in the organization when the Gender Mainstreaming Strategy 2008-2012 (GM Strategy) was issued and put in place. Most informants recognized how the gender issue had moved from the side-line to the centre of development work. The emergence of the GM Strategy originated from the findings of a quick assessment, which showed superficial understandings of gender among many staff. This low understanding undermined the quality of many on-going projects (Doc.3). INGO 1.1 viewed the strategy as a great step towards institutionalizing gender mainstreaming in the whole organization. According to all informants, the Strategy was an essential reference point for those who were directly or indirectly involved in gender work with an aim to consistently integrate gender concerns into programmes. It provided a firm basis for resources to be dedicated to fulfilling that mission as INGO 1.1 believed. 
The GM Strategy introduced basic concepts such as gender, gender prejudice, gender roles, gender needs, gender equity, gender mainstreaming, and gender equality. It included three main objectives: to strengthen staff and partner understanding of, and capacity to promote, gender equity; to improve organizational structure to support gender-based programming; and to broaden the evidence and advocacy base for promoting gender equity in policy and practice. It was clear that the GM Strategy stressed the importance of gender as a priority area to improve projects' quality. In the GM strategy, the need to re-invest in the Gender Task Force (GTF) as gender machinery in this organization was addressed. The GTF was aimed at facilitating the gender mainstreaming process both in office and at project level. In addition, the GM Strategy was very explicit about the integration of both women and men in the whole project cycle from the planning stage, the design stage, the implementation stage, the monitoring stage, and the evaluation stage. Simple assumptions that women would automatically benefit from any given project or programme were discouraged. (Doc.3)

The organization strived to build a close link between the GM Strategy and its core principles of empowerment, accountability, partnership, non-violence, non-discrimination, and sustainability. The GM Strategy must be rights-based, evidence-based, and communitybased. In this Strategy, key activities were designed to achieve the above-mentioned objectives, which sought to sensitize staff to the importance of gender equity and equip them with the tools and skills required for gender equity promotion, set up mechanisms to monitor and evaluate practices of gender work, mobilize resources to implement the Strategy, and draw lessons from piloting gender mainstreaming in some projects for wider dissemination and advocacy (Doc.4).

\section{* The Gender Taskforce (GTF)}

The GTF was launched in the organization in 2007 to assist the process of gender mainstreaming based on the recognition of the need for internal capacity building to fulfil core principles of equity and empowerment. The GTF consisted of ten Gender Focal Points (GFP) who were national and international staff, representing all programmes as well as human resources and finance departments. GFP were individuals representing diverse 
gender, a diversity of experience with gender work (beginner and advanced), and a diversity of staff positions. The original list of GTF members included two men and eight women. Five of them were based in Hanoi and the rest were in Ho Chi Minh City and other provinces. Two out of ten members were from Human Resource and Financial departments whereas the other eight people represented various programmes (Doc 4).

All GFPs were responsible for helping disseminate information and provided technical assistance for gender mainstreaming in their programmes, projects, or otherwise assigned roles. It was expected that each GFP would dedicate approximately 10 percent of their time to the GTF's activities. This was written in their job descriptions and assessed through annual appraisals by their supervisors. An annual work plan for the GTF was developed and each GFP had a specific action plan in case their project or programme was selected for gender mainstreaming. All GFPs were anticipated to participate in Skype or telephone conferences held every quarter, GTF training courses, gender orientation session facilitation for staff and/ or partners, periodic gender knowledge and skill assessments, annual planning for gender mainstreaming, individual action plan activities, and external gender events. The Gender Equity and Diversity (GED) and Learning Coordinator was expected to monitor and support GTF efforts. This individual was responsible for reporting to the Deputy Directors of Programme on all gender mainstreaming efforts under the organization's programme quality framework, achievements, and challenges. The following Figure illustrated supervision lines. (Doc4) 


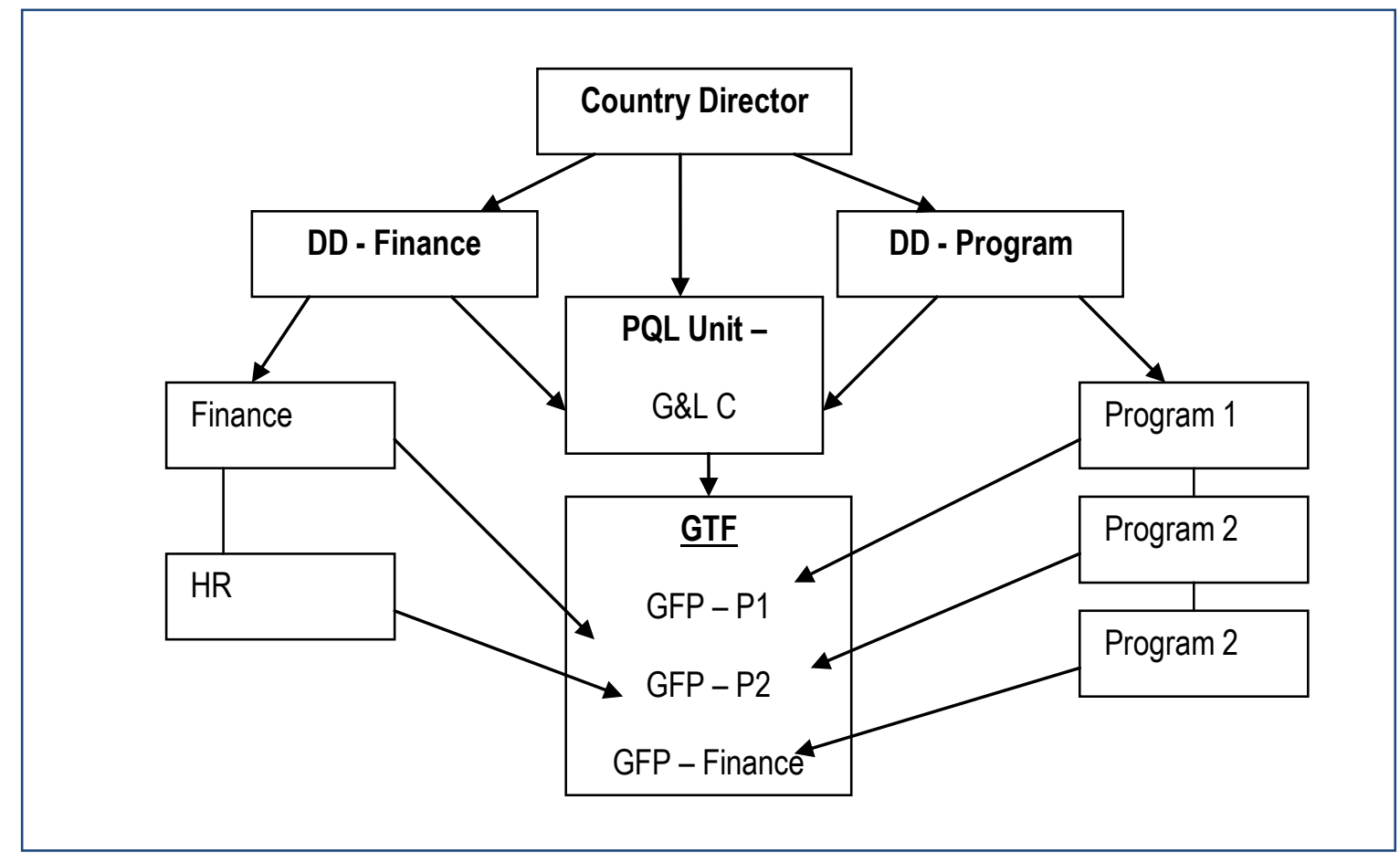

Figure 5.1. Supervision lines of the GTF in the INGO (Source: Gender Task Force, Term of Reference, issued by the INGO, 2008)

\section{Gender Equity and Diversity and Learning Coordinator}

The GED Coordinator was nominated in 2008. He was expected to work with and provide assistance to senior programme staff and project teams to promote a learning environment and enhance programme quality, in line with identified priorities. This position was also responsible for supporting a programme-wide GED Task Force; developing a sound base of GED competencies among programme staff, and supporting access to and application of practical tools on GED for staff and partners. Specific duties of this position were to coordinate the development of a Gender Strategy in consultation with senior programme's staff and GTF members; activate and support the operations of the GTF including capacity building for GTF members, and supporting the development of effective GTF plans and activities; ensure effective GED capacity building in staff orientation; develop a GM Toolkit; support programme teams to effectively integrate programmatic gender mainstreaming at programme and project levels, including through providing hands-on support and coaching; ensure effective access to appropriate Gender indicator toolkits for programming staff; participate in design, monitoring and evaluation processes of projects as required to ensure 
effective gender approaches; represent the organization externally and liaise with other organizations; and stay abreast of the organization's gender initiatives (Doc. 5).

\section{c) Human Resource policies from gender perspective}

Most informants unanimously agreed that the INGO was a comfortable work place, in which female and male staff enjoyed equal policies and incentives. The flexible working hour was cited by INGO 1.2 and INGO 1.4 as evidence. INGO 1.2 - Human Resource Manager reported that incentives were designed to create the most favourable conditions for employees' performance at work while handling their private lives satisfactorily. INGO 1.2 named examples such as the provision of one-to-two hour breaks for breast-feeding and the possibility for baby and a baby sitter to accompany staff on business associated with the organization. Similarly, INGO 1.2 made it clear that male staff could also choose to take one week off before or after his wife's baby delivery. All the maternity policies were happily affirmed by INGO 1.4 who was breast-feeding her son at the time of interviews.

All informants highlighted that there was no gender discrimination in job opportunities, promotion, and development in the INGO although the gender ratio in the organization was not perfectly balanced, with a higher number of female than male staff. INGO 1.2 thought that this could be common for NGOs in Vietnam. The organization, however, had no intention of achieving an equal ratio of men and women by prioritizing women over men in the recruitment process because, according to INGO 1.2's explanation, a candidate was selected on the basis of her or his capacity and ability relevant to the requirement of any given post, rather than on sex. Moreover, being a male or a female did not hinder them from being promoted or developed at work (INGO 1.2).

INGO 1.2 also admitted the difficulty facing the INGO and other organizations in employing more male employees. INGO 1.2 thought the reason for the absence of male staff was simply because they were not interested in the development sector. She also worried about achieving an equal gender ratio in the organization. The gender disparity was found to be more unbalanced at programme coordination level and at project level in the staffing structure. INGO 1.5, for instance, reported eight male coordinators and only one female 
coordinator. No women were working for forestation projects because the family burden prevented them from travelling much to the field (INGO 1.5).

\section{d) Improving technical capacity of staff in gender}

At this stage, the requirement for gender knowledge and awareness has not been written into job requirements or job descriptions (INGO 1.1). However, for some positions working for projects, candidates had to show a certain level of gender knowledge and awareness (INGO 1.2). Or rather, they needed to express appropriate attitudes towards gender and care about gender, or at least women's empowerment and participation in decision-making (INGO 1.5). All informants summarized the gender training process for staff in the organization as follows. Once employed, staff were asked to take an orientation course, which was mandatory to all staff. This course was held every six months to provide new staff with basic concepts, knowledge, and awareness of gender issues together with other focus areas of the organization. INGO 1.1 added it was a chance for all staff to understand how much gender was of concern in the INGO. Intensive trainings were organized for programme coordinators, project managers, project staff, and GTF members, in order to impart deep understanding of gender, gender analysis skills, and gender integration into project activities (INGO 1.1).

\subsubsection{Institutional challenges caused by a superficial and inconsistent process}

The GM strategy was issued in 2008 as a common reference point for everybody working on gender mainstreaming in both the office and in field projects. This document created the basis for the organization to make both human resource and financial investments so that overall objectives could be achieved eventually. After four years of putting it in practice, some positive results were praised by some of informants. They were: more favourable working conditions for female and male staff; improved gender awareness and knowledge of all staff; better gender analysis skills and gender action planning; and funding for gender mainstreaming efforts. However, the process was blocked when the ILO changed its institutional framework from gender mainstreaming to women empowerment in early 2011, as INGO 1.1 confessed. The change in the working framework made by the ILO confused the organization. In other words, the INGO had to deal with a dilemma of continuing gender 
mainstreaming process and being in line with newly adopted women empowerment framework (INGO 1.1). This made one challenge for gender efforts. The other challenges are named in the following sections.

\section{a) Lack of understanding of and capacity for doing GM}

\section{* Confusion in understanding key concepts}

Fundamental concepts such as sex, gender, women's empowerment, and gender equality became familiar to people in the organization. They could talk about, understand, and distinguish these notions quite well. However, people remained confused about the definition of gender mainstreaming in theory and in practice. All informants knew gender mainstreaming in projects to be a process of integrating gender concerns in the whole project cycle from design, implementation, monitoring and evaluation. However, some of them simply understood gender mainstreaming in the organization itself as having more privileges for women and/or getting more women involved in the activities of the organization, or they misperceived gender equity and gender equality. In many cases, there was a high possibility for people to conflate gender issues with women's issues in the organizational context. A following segment of interview with INGO 1.3 illustrated this well:

Ah, difficult, isn't it?! [GM] seems easier in projects. For example, in common activities like meetings or work group division, we always try to have equal gender representation in a meeting. Or for example, one project reflects having too many men, so they have a strategy to invite more women into that project. People also reflect that having women's involvement makes that workgroup more moderate, easy going... In daily work, I do not know whether it means gender mainstreaming or not but the majority of staff are women but they are very strong, mostly equal. Men always respect women although they are fewer, in common activities or meetings...

INGO 1.2, as an HR Manager-cum-GTF member, seemed genuinely uncertain about how gender mainstreaming should be implemented in human resources, financial, and other organizational policies. HR policy-making always took account of women and men to make sure that they were enabled to work under as favourable conditions as possible (INGO 1.2). However, it made no sense to INGO 1.2 to write a chapter on gender equality in HR policy 
without specifying what it meant in real action. She recommended hiring an external consultant or a more experienced person to help with that.

\section{* Lack of continuous and in-depth trainings}

INGO 1.1 observed that everybody in the organization had basic knowledge and awareness of gender. As noted by all respondents, those working for projects were trained more intensively to use essential tools and skills for gender analysis, designing gender-sensitive indicators and their incorporation into project activities. The transferability of knowledge into action, however, remained challenging to staff because of the lack of continuous and indepth training. Training for staff of human resources, financial departments and non-project units was a one-off activity in the INGO. There was "no fixed schedule for gender training" (INGO 1.5), or it may happen irregularly (INGO 1.2). INGO 1.3 received her latest gender training in 2008. The staff's time budget and capacity may be limited as the GED Coordinator (INGO 1.1) explained. This lack of frequency may lead to the failure of sensitizing all staff of the organization to gender issues in their daily work.

\section{* Unavailability of practical guidelines/tools:}

The GM Strategy specified very general objectives. It was understandable for INGO 1.3 to have forgotten the document altogether. I also understood why INGO 1.2 cried for practical applications, particularly for gender in human resource policies when theoretical material like the GM strategy seemed abstract and daunting to people with high workloads. Training seemed inadequate. While there were Gender Mainstreaming Guidelines, these were still in draft form, and the organization was not able to promulgate it for reasons identified by INGO 1.1. Firstly, there was not enough money to implement these guidelines properly. Secondly, human resources or GTF were not strong enough to facilitate such a process. Lastly, the women empowerment framework launched by the ILO temporarily stopped gender mainstreaming. As a consequence, all top leaders and those responsible for gender in the INGO had to seek the best way to interweave the current GM strategy with the latest ILGO framework. As this change happened at the time of my interviews, I observed firsthand INGO 1.1's frustration and the physical exhaustion created by the pressure that such interweaving brought. 


\section{- Limited capacity of some staff}

In the Terms of Reference (TOR), the GED Coordinator was required to carry out a number of different tasks which were mainly aimed at promoting gender mainstreaming in both the office and in projects. As in his TOR, a relevant qualification and experience was a must for this position. However, this Coordinator started the job with a less than basic level of gender knowledge as he himself confessed. INGO 1.1 did not hesitate to unveil his background in healthcare, rather than gender, presenting in his work history that he had not worked closely with gender issues before he took this position. He told me about his efforts to undertake the job as professionally as possible, resulting in his improved gender knowledge and skills over time. His attempts have received high appreciation from colleagues who commented on his important role as "a motivator to keep things moving on" (INGO 1.4) and on his aim "to build connection amongst programmes in regards of gender mainstreaming" (INGO 1.5). Nevertheless, his capacity remained limited to a degree, as INGO 1.5 reflected. INGO 1.5 explained that the GED coordinator's advocacy might be valuable to a healthcare project but less so to a forestation or a rural development and agriculture project (INGO 1.5). Thus INGO 1.5 argued that while INGO 1.1 was an expert in gender, he was not an expert in another focus area. In this sense, a forestation specialist was thought by INGO 1.5 to better understand what a woman could be expected to gain from that kind of project activity. This finding suggests that while being an expert in gender is a necessary condition for the job, it is not a sufficient condition alone to fully meet the demands of the job; knowledge of other fields is also needed from a GFP staff member.

All informants concurred that gender analysis was the first priority in the whole gender mainstreaming process. However, the lack of updated and in-depth training was thought to be primarily responsible for the limited capacity of some other staff to do this. As INGO 1.5 indicated, "not all staff of the organization are trained to properly integrate gender in their works". INGO 1.5 believed that project staff were able to define gender analysis and the tools best suited for it in order to draw an overall picture of gender needs, gender issues in a given project site and the involvement of men and women in the project. However, they did not know how to use the results of their analysis (INGO 1.5). In other words, they were not able to integrate the results from gender analysis into the specific activities of a project and 
improve gender equality. INGO 1.3 - a project officer of a climate change programme demonstrated this limitation by revealing her lack of confidence in her own gender analysis capacity. She said, "we have the feeling we do not really deeply analyse gender issues in our climate change programme". Because of this, she raised her demand for training in terms of quantity and quality. INGO 1.1 and INGO 1.5 also mentioned the need to provide staff with better understanding and skills. At this stage, INGO 1.5 suggested gender analyses be done by those qualified on the programme's staff. According to INGO 1.5, these staff, as actors in a specific sector, knew the requirements of their job. In addition, they had direct communication with local people and could understand the needs of people. The possibility of enforcing his recommendation, however, depended on three factors, including donors, funds, and the time budgets of projects and staff. These factors were all found to be problematic.

\section{b) Lack of commitment and accountability from all stakeholders in practice}

\section{Gender being out of focus for local partners}

According to INGO 1.4, the INGO chose to gradually let local partners implement all projects. As such, the organization provided just technical assistance and training to ensure every stakeholder had common and consistent understanding of the organization's focus areas (INGO 1.4). To achieve this, the INGO often met with partners to discuss the issues concerned. Nevertheless, gender was not yet on the table for discussions in these meetings because, as INGO 1.4 noted, "partners have to prioritize other tasks other than gender work". INGO 1.4 was managing some projects which began with training on the Gender Equality Law and Anti-domestic Violence Law delivered by local partners. This is to say that, to a certain degree, gender issues remained at the margins of both the INGO's and partners' attentions. The lack of understanding and knowledge about gender mainstreaming processes could be a reason for partners' downplaying gender work in favour of other tasks. Local partners were also unsure about the significance of gender integration and/ or the consequence of excluding this in their projects (INGO 1.5). Given this reality, there was a danger that partner organization put less weight on gender issues than other activities on the partner's side. 


\section{Donors}

The INGO received funds from various donors, and hence its operation was, more or less, driven by donors' decisions. As INGO 1.5 observed, not all donors considered gender analysis as a focus point in their projects. INGO 1.1 highlighted that donors paid attention specifically to climate change, HIV/AIDS or concerns other than gender. There was not much potential to appoint gender focal point persons amongst programme staff to carry out gender analysis as suggested by INGO 1.5. Conversely, if the donor let staff undertake this task, then she/he would have the double burdens of daily work and extra gender work (INGO 1.5). If that was the case, the feasibility of this option, therefore, would be low due to working pressures and time constraints. Moreover, there were tightly time-bound project activities - the term of a project is only a few years (INGO 1.5). Deadlines came quickly after one another, so that gender mainstreaming was often missed at some point (INGO 1.4).

Gender issues have drawn great attention and concern in the development sector. All development donors have adopted it as a mandatory part of projects or programmes. In other words, it is an indispensable point in a checklist to be ticked off before approving funding for any specific project. This checklist makes sense if it is done exactly as expected. However, the practice, as it appeared in the INGO, seemed to be far away from the theory with some informants claiming donors had varying degrees of attention to and budget for, accountability and mainstreaming of gender concerns. For some donors, it was a fashion to include gender concerns in every proposal, as a line or a paragraph to be ticked off (INGO 1.1). INGO 1.5's programme had a Danish donor, for example, which put gender issues on top of its priorities. However, this specific donor was more concerned with women's involvement: "As a rule, Denmark is not funding anything if they do not see presence of women in that project" (INGO 1.5). The other finding was a lack of harmonization amongst the donors of the INGO in terms of gender work: as INGO 1.5 explained, his programme started to provide gender training before the year of 2008. Private donors have gradually become an additional source of funds for the INGO's activities but gender was not their concern (INGO 1.5). In INGO 1.5's experience, funding was not usually made available by all donors in order to invest more human resources in the gender work of a specific programme if needed. Moreover, donors continued to view gender as a persistent or unsolvable issue, 
which could not be addressed by a single project or programme (INGO 1.5). It may be true in a way. However, this thought created delayed responses and discouraged accountability for gender outcomes. Instead, INGOs were required to produce evidence of women's participation in project activities. Despite determination to step forward with gender mainstreaming, the INGO had little choice but to be driven by donors' priorities as a conditionality of aid and hence produce WID outcomes.

\section{* In the organization - staff and leaders}

Since gender was identified as one of the catalysts for the improved quality of project delivery, all informants recognized the goodwill of the leaders of the organization for gender mainstreaming efforts, and "being supportive to whatever things aimed to facilitate gender mainstreaming process" (INGO 1.5). These respondents noticed succinctly that the nomination of a fulltime GFP, rejuvenation of the GTF, provision of gender training opportunities at home and outside for staff, and allocation of funds for gender-related activities illustrated support at the management level.

According to INGO 1.4, gender was brought to almost all meetings at an organizational level. Nonetheless, there were some concerns. First of all, highly detailed technical understanding of gender work was thought to be absent amongst leaders (INGO 1.5). Secondly, INGO 1.5 claimed that the current amount of funds seemed insufficient to implement gender mainstreaming effectively and efficiently, while additional human resources and funds were needed to support gender tasks in communities. Indeed, gender work remained an add-on activity in terms of financial commitment (INGO 1.1). INGO 1.1 presented some cases in which gender work was supported by the leftover funding from other activities or an amount that was insufficient for other activities. The lack of resources was thought by INGO 1.1 to be a part of the reason why the GM guideline had not been issued to date. As INGO 1.1 stated, the "strategy for fund allocation for gender work is essential. The current amount is very small". Thirdly, INGO leaders proved not to be held explicitly accountable for the gender outcomes in their internal systems or in their projects. According to findings, not only secondary senior staff but also top leaders in the organization did not raise questions if gender work was unfinished or not properly done in projects (INGO 1.5). Apparently they put more weight on other activities such as increased incomes for the poor, because gender, as a 
persistent issue, could not be addressed (INGO 1.5). INGO 1.5 made a serious complaint about the organization's accountability to gender work as "a weak point". He thought it was everyone's and yet no one's responsibility in the organization.

... we feel okay if gender work is reported unfinished or not done well... we talk a lot about gender but we do not pay enough attention to it. Everything is just limited to theory. ...I have never seen any staff being blamed or complained [about] if GM is not done well.

INGO 1.1 also felt that some in the INGO still viewed gender work as a voluntary task and did not include it in their daily responsibilities. A part of the reason for this was that gender work was not specifically written into job descriptions at any level or in the Annual Performance Appraisal (APA) process (INGO 1.1).

\section{c) Insufficient attention to organizational culture}

As mentioned in the first part of this data analysis, the organization created a strong foundation from which to gradually step into a gender mainstreaming process. There was strong backing from management at all levels of the organization and the release of funding and time for staff to engage in issues related to gender. The organization was reported to have no gender prejudice (INGO 1.1), no gender bias in decision-making power (INGO 1.5), and no bureaucratic hierarchy (INGO 1.3) at all levels. Put another way, a workplace of maledomination was thought not to exist here. According to all informants, everyone felt free to speak their mind and be heard by their colleagues. They believed that female and male staff were equally respected and involved in the organization's activities and meetings while hierarchical structure did not appear to be a barrier for people to share their ideas and take part in decision-making processes. Although the number of female staff surpassed that of male staff in the office, they often held deputy positions in the organizational structure. INGO 1.1, for example, shared that the top leader was male and two deputies were female. In the second top leadership level of the organization, there was only one woman out of eight men. Regarding equal power in decision-making, INGO 1.1 said, "...in this level, women are not left out but well...", then hesitated for a while before finishing his sentence, "...but just only one woman..." 
To a significant degree, gender became a topic of both casual lunch talk (INGO 1.2) and discussion in formal meetings in the organization (INGO 1.4). Gender was put on the agenda of all meetings at all levels. However, staff commented that while talk of gender was ubiquitous, "sharing is not deep at all" (INGO 1.5). The lack of depth in this activity may be attributable to the aspect of Vietnamese culture (INGO 1.5) whereby people expect to learn from teachers but rarely from friends or colleagues. From INGO 1.5's observation, when two teams were brought to a sharing session about the experiences of gender work, one team always viewed itself to be better than the other. People hesitated to be critical of others or to offer help to others because, as a part of Vietnamese culture, questioning was uncommon or, as the local saying runs, "speech is silver, silence is golden" (INGO 1.5). On the other hand, participants in this session may raise the sort of questions that were considered to be thorny in other cases. In the spirit of showing off, people barely learnt from each other and thus opportunities for improvement are lost. Learning and sharing sessions or personal reflections were thought to be useful but under-valued at this stage. As such, they now only seldom occurred (INGO 1.3 and INGO 1.1). Another idea - to gather husbands and wives of all staff in a retreat to talk about gender in a tactful and sensitive manner - has been on hold because the size of the organization made for logistical impossibilities (INGO 1.1).

\subsubsection{Gender Mainstreaming or Women Mainstreaming}

Looking back to the time before 2008, INGO 1.5 said the organization used to focus on just increasing the number of women in project activities without considering their needs and interests. The year of 2008 saw a change to that trend with the emphasis shifting to both men's and women's issues. Accordingly, the GM strategy was issued to provide the institutional basis for the gender mainstreaming process. Gender mainstreaming was a new concept which was confusing to the organization. The organization has now experienced the transition to the new approach of addressing gender issues but has tried hard to establish a fundamental foundation of knowledge, skills, and budget. It has also piloted mainstreaming of gender concerns at the implementation stage of some existing projects. This process has met several difficulties: 


\section{a) Impasse of the designed project}

All respondents were aware that the organization was piloting gender mainstreaming in some projects at the implementation phase. However, some projects were designed without gender consideration while target beneficiaries were fixed (INGO 1.1). In these project documents, communities' issues were assessed but women's and men's issues were not taken into consideration. Thus, "there is no single sentence written about women's issues" (INGO 1.1). For these projects, gender analysis at the implementation phase became very important in order to adjust project activities and ensure that gender could be integrated into specific operations (INGO 1.1).

\section{b) Gender in Project Implementation}

For already-designed projects, the organization attempted to involve women or at least not put women at the margins of the project (INGO 1.5). According to INGO 1.1's information, the current model of gender mainstreaming in other organizations was limited to attracting more women and to improving their capacity. The INGO struggled between wanting to do more and being aware of the need for more time and resources, as INGO 1.5 realized. Some programmes, such as rural development and agriculture considered gender concerns in their projects from 1997, as INGO 1.5 mentioned. Gender training for the programmes' staff and stakeholders were commenced in 2006 (INGO 1.5). In this programme, INGO 1.5 and his staff admitted the difficulty and complication of carrying out gender mainstreaming in compliance with guidelines even when they were trained to do so. In INGO 1.5's opinion, more would be possible if human resources were doubled. Currently, the GTF seemed not to be functioning properly, evidenced by the fact that only the GED coordinator remained (INGO 1.5). The organization intended to appoint more senior staff to this unit with the hope that they would make longer commitments (INGO 1.5).

INGO 1.3 was engaged in a project of natural resource management and livelihood. In the framework of this project, gender analysis was conducted to identify gender needs and the gendered labour division in project sites. Accordingly, interventions were designed in response to men's needs and women's needs. In many cases, activities sought to increase women's participation in the project (INGO 1.3) as people remain concerned about how to 
attract more women into that particular process (INGO 1.5). Similarly, the target beneficiaries were women alone in a new project managed by INGO 1.4. In this project, gender mainstreaming has not yet been given much attention from stakeholders. Hence, only a training course on gender roles and gender equality law has been provided to local collaborators to date (INGO 1.4). In other cases, such as, for instance, the one INGO 1.5 outlined, "from 2007-2009, gender mainstreaming has been implemented in some projects. However, there are some projects specific to women only. I see that these projects empowered women very much". The staff were required to report on the increased participation of women in the project as a prerequisite condition. Otherwise, the staff were reminded to involve more women and keep this in mind (INGO 1.5).

\section{c) Gender in the Monitoring and Evaluation system}

According to the desk review, a Monitoring and Evaluation (M\&E) system for gender work was unavailable in the organization. In many cases, respondents reported the outcome of gender mainstreaming efforts from their own experience (INGO 1.1). In the INGO's structure, programme coordinators and project managers were in charge of ensuring the project's quality, including gender integration into projects (INGO 1.5 and INGO 1.1). This aspect has been written in their job descriptions and was not the responsibility of a gender coordinator (INGO 1.1). For example, INGO 1.1 said, "I may not have official assessment but my feeling is that the majority of them have good awareness, knowledge and commitment to gender work".

\subsubsection{Opportunities for INGO's greater contribution to gender equality in the long- term}

\section{a) From individual change to social change}

According to some informants, traditional and cultural norms continued to affect women's career paths. Family burdens prevented women from valuable opportunities for job promotion and development in their work place (INGO 1.2). In this organization, talking about gender relations at home was a casual activity every day in which female staff expressed their triple-burdens of full-time working, domestic work, and child-rearing. INGO 1.2 repeated that she was fortunate to have a husband who was willing to share housework, 
take care of children at home, and respect her in family decisions. She believed that her husband's working for an NGO in Vietnam, had made a remarkable contribution to forming his view in this regard.

An interesting finding was that men working for the development sector had a tendency to be open and advanced in their minds and attitudes toward gender roles at home. Male staff in the INGO were complimented by their female colleagues as being industrious in regard to housework and rearing children. The explanation given by INGO 1.2 was that almost all NGOs had gender work - or at least an orientation about gender for all staff - which gave them better knowledge and awareness of gender issues. All informants shared the common viewpoint that working for NGOs could have a positive impact on this significant change. Moreover, initial resistance to a man in the role of GFP staff was gradually replaced with the realization, and high appreciation, of his particular strengths in the organization and development community (INGO 1.1). INGO 1.5 impressively concluded that "all women in the organization wish to marry men working in the development sector". The daily casual sharing of staff about these aspects of their work and home lives acted as an impetus for the organization to organize a retreat involving staff and their partners in which gender would be talked about (INGO 1.4). To date, this has not happened but if this idea could be brought to fruition, it would help to negotiate changes to people's attitudes and perceptions of gender.

\section{b) Capacity-building for a grass-root level}

In Chapter 3, the legal frameworks and institutional machineries in Vietnam for gender were mentioned to demonstrate the Vietnamese government's determination to address gender inequality. According to INGO 1.1, the translation of laws into real life appeared inconsistent from the central level to the grass-root level. Therefore, INGO 1.1 believed, through partnerships, the INGO was contributing to disseminating the contents of these laws, and to building and developing gender consciousness, awareness, knowledge, and skills with local partners. Local partners in this instance are State organizations, other social organizations, local governments, and technical organizations (INGO 1.1). 
The INGO's appointment of a man working as a GFP made people realize the role of men in promoting gender equality. In addition, there was potential for the INGO to increase local government commitment to gender concerns (INGO 1.1). INGO 1.1 acknowledged the change in the local partners' understanding of what counts as gender equality. Yet, thanks to the cooperation with the INGO, local partners learnt to analyse gender issues and incorporate the results into gender action plans. The contribution of the INGO in these respects becomes more urgent than ever, if all State officials are to be equipped with appropriate awareness and knowledge of gender issues as required (INGO 1.1).

\subsection{Summary of findings and data analysis}

Referring to the International Gender Policy issued by the ILO, the INGO in Vietnam officially launched gender as one of its focus areas from 2008. Previously, gender integration had already been introduced in some programmes - such as rural development and agriculture while the other programmes concerned women's issues in isolation, or were gender blind. Hence, 2008 was the moment when gender incorporation became an inclusive requirement for all activities in the office and in the community. Once making gender a key focus, the INGO made significant investments in the technical conditions needed to kick off the gender mainstreaming process. These conditions included the nomination of full-time GED coordinator, the establishment of a Gender Taskforce, the issuance of the Gender Mainstreaming Strategy, gender training provision for staff, building capacity, skills of gender work for staff, designing staffing policies from a gender lens, and a budget allocation for gender work, M\&E, accountability, and organizational culture. The following diagram draws out these inputs as measures of the organization's preparedness for implementing gender mainstreaming, and speaks to the eventual aim to institutionalize gender in the organization as a whole. 


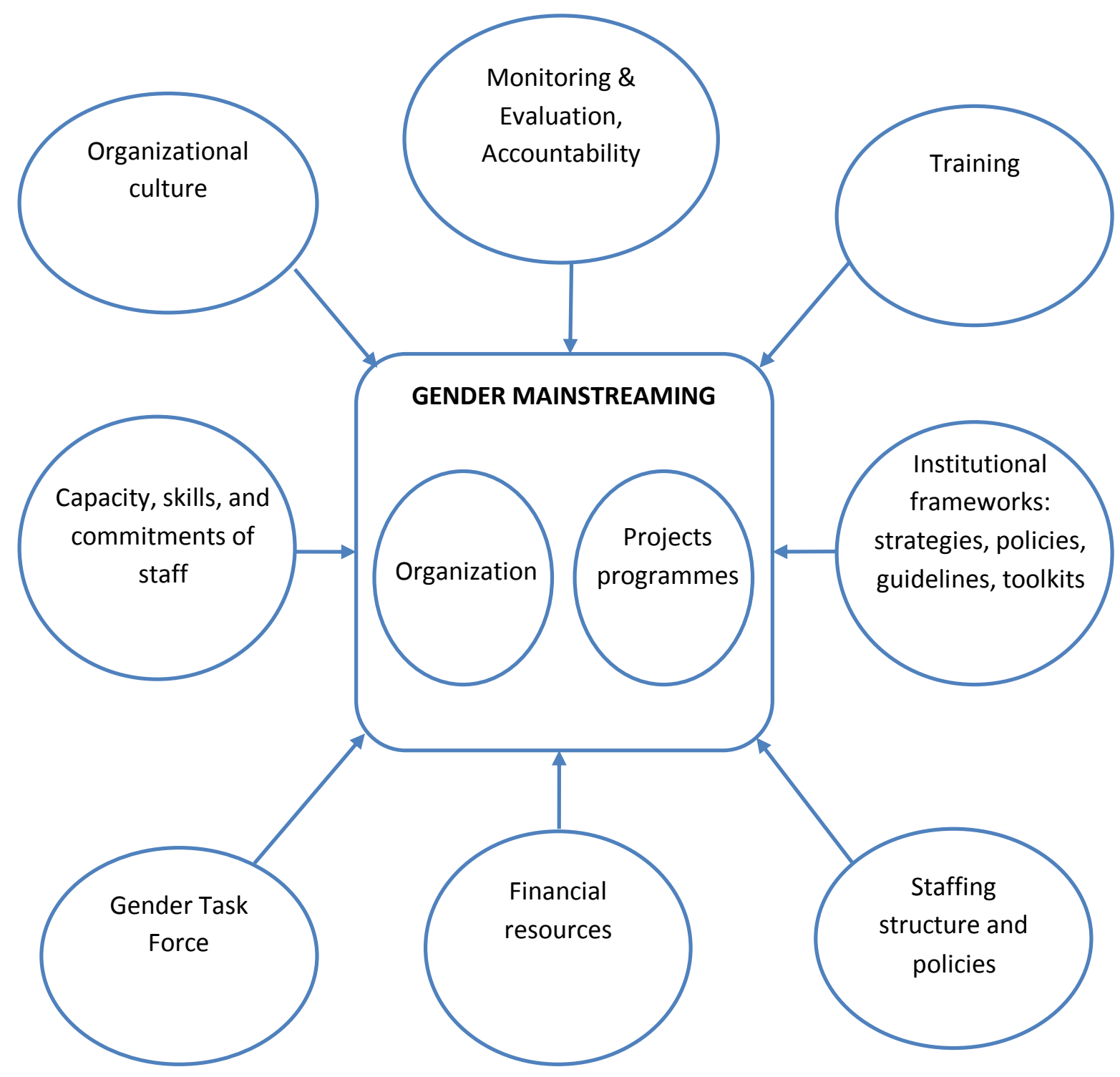

Figure 5.2. Technical preparedness of the INGO to take off the gender mainstreaming process

The technical conditions summarized in Figure 5.2 have already taken place in the INGO. If this is a checklist, the findings show clearly that the organization has ticked off all points. The organizational investments, however, appeared to be problematic and limited within the organization. In the first place, it seemed that the language remained confusing to some, while others oversimplified the notions underlying these terms. According to findings, it was likely that the word 'gender' was spoken when the term 'women' was meant. Apparently, people in the organization easily conflated gender with women. Letting alone the implementation, this obstacle raises technical questions around the whole process. 
Other impediments to the organizational capacity for gender mainstreaming include:

(1) Infrequency of - or no - in-depth training;

(2) Limited technical capacity and skills of all staff at all levels;

(3) High staff turnover, lack of human resources, and unbalanced ratio between men and women in staffing structure;

(4) Insufficient budget;

(5) Malfunctioning of Gender Taskforce;

(6) Lack of institutional frameworks;

(7) Absence of M\&E and Accountability mechanisms;

(8) Lack of attention to the importance of organizational culture in challenging gender norms in a wider society

The process of gender mainstreaming in the INGO became more challenging than ever when the INGO was forced to negotiate between the respective positions of the ILO and their donors. Figure 5.3 below illustrates the power relations in the decision-making process, which could perhaps be described as 'two nooses - one neck'. Literally speaking, the INGO had to depend on donors' decisions as a conditionality of aid while strictly complying with the ILO's instructions. The difficulty of this became noticeable when there was no harmonization on focus areas, funding, and approaches to gender work amongst donors. It led to inconsistency in the gender mainstreaming process between different programmes and projects. In addition, the change in gender policies or strategies made by the ILO affected the progress of gender mainstreaming in the INGO. For example, the ILO shifted its orientation from being gender-focussed to a focus on women's empowerment. The organization became highly concerned that it deal with, adopt, and adapt to the current context of the organization and Vietnam. As demonstrated in the below diagram, this topdown mechanism might cause more confusion and inconsistency: when decisions were decentralized, local partners were found to have limited capacity, and a tendency not to focus on gender work. All these may result in fewer opportunities to generate gender outcomes in communities at the end. 


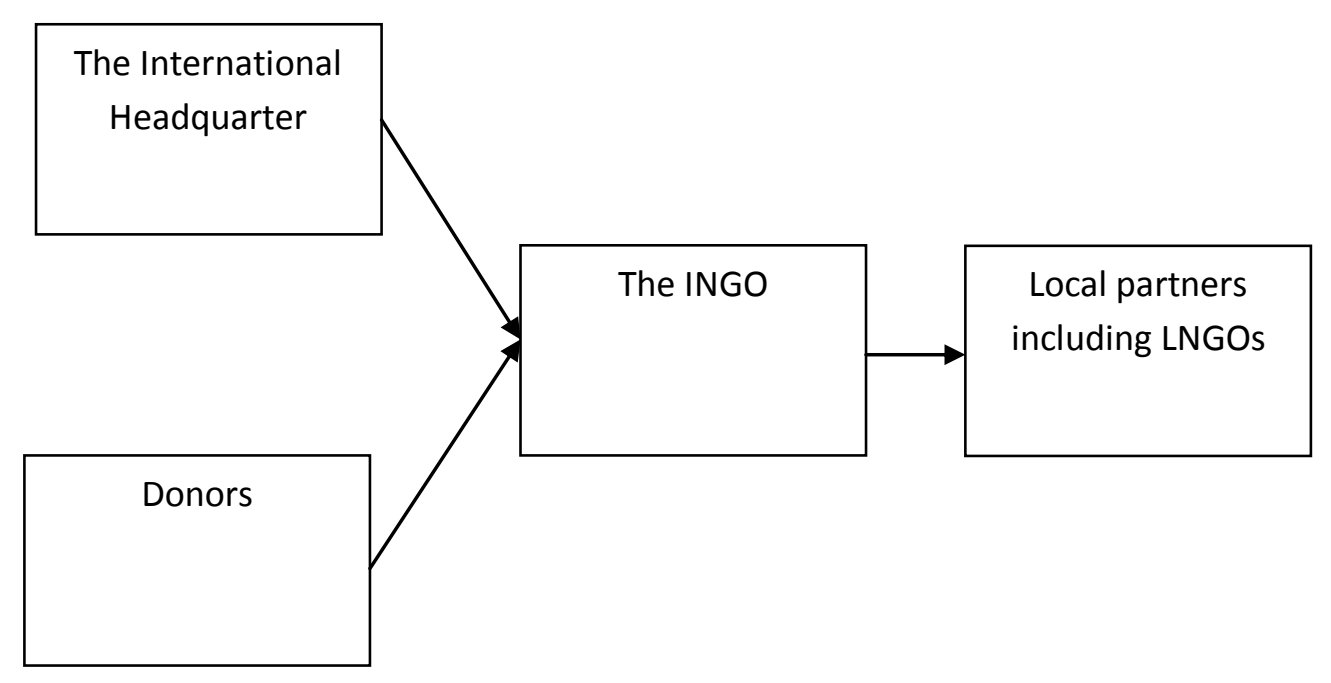

\section{Figure 5.3. Power relations in decision-making process}

Going against all the constraints listed above, gender mainstreaming efforts made by the INGO have still gained some significant achievements which have helped to move the INGO closer to the first level of the ladder in Figure 5.4 to the goal of gender equality:

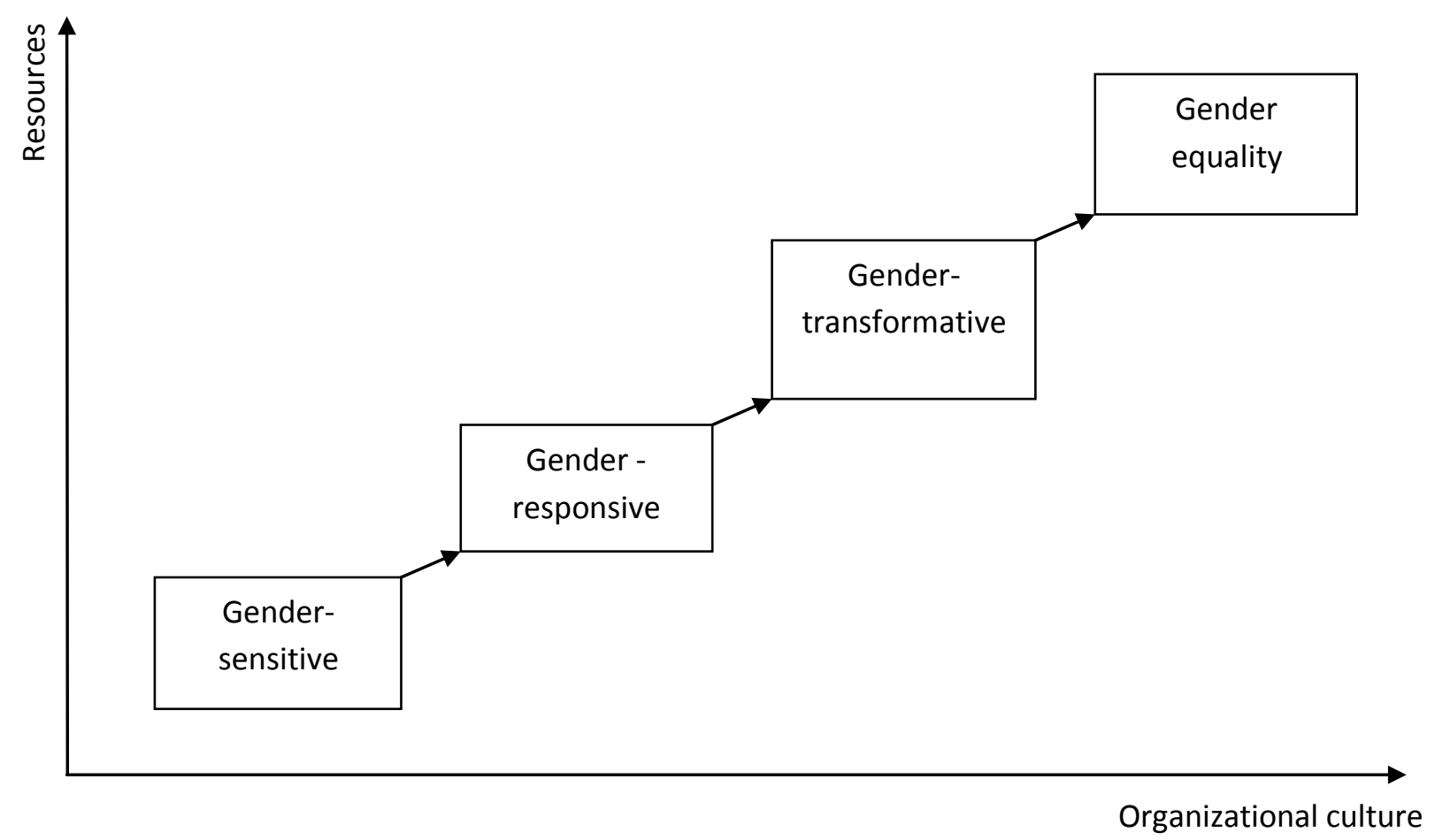

Figure 5.4. The progress to gender equality and factors influencing that progress

The development of the GM strategy in 2008 drew significant attention from the organization and made them strive to incorporate it into their daily office and field work. The 
top leaders strongly backed this process while staff expressed their goodwill towards gender integration. Thanks to the INGO's investments, staff became more aware and knowledgeable about gender issues and mainstreaming. Notably, some programme and project staff learnt how to carry out gender analysis and used the results for activity design and implementation. Gender mainstreaming had been piloted in some on-going programmes and projects at the implementation stage to learn lessons for designing the upcoming projects. The INGO chose the method of "learning by doing". INGO 1.1, as GED coordinator of the INGO, assessed the organization to have passed the first level - "gendersensitive" - and progressed to the second level - "gender-responsive" - of the ladder. I, however, thought that the organization still remained at the edge of the first level as my findings suggested inadequate human and financial resources had been invested, while a lack of heart-felt and relevant interventions to challenge the male-dominated culture and norms embodied into the organizational culture from society still seemed evident. Consequently, the organization has not progressed further in the gender mainstreaming process.

Needless to say, however, it takes time and energy for an organization the size of the INGO to change regimes. Presently uncertainty surrounds which approach the organization is using for gender work: WID or GAD or a mixture of the two. Gender mainstreaming is internationally appraised as the ideal approach by which the dominant GAD theory may be translated into practice. INGO 1.1, as a GED coordinator, believed that GAD was how the INGO chose to address gender issues. The finding, however, showed that the main focus continued to be on women's issues and taking account of their participation, rather than gender issues and power analysis in relation with other social factors. Within the organization's human resources policies, for instance, support for women and the handling of their issues outweighs the equivalent support offered men. In communities, reports on gender outcomes are always limited to discussion of the rate of women's involvement in activities. At the moment, there is no clarity around how the choice between adopting WID or GAD is being negotiated, or if both approaches are regarded as interchangeable and supplementary to each other. 
Finally, it is crucial to highlight the finding around the lack of in-depth sharing in the organization, and between development sector organizations, in Vietnam as part of Vietnamese culture. The GED coordinator's interest in this research was partially explained by his anticipation of accessing insights into other organizations' gender work. On behalf of his organization, he was hoping experiences and lessons could be shared by and with other institutions in order to find the common route to improve the status quo of gender issues in Vietnam. In fact, this point is missed in the development sector in Vietnam. This also reflects the situation within the organization in which staff spoke widely but not deeply about gender work. This raises further questions around the coherence and sensitization of gender work in the INGO.

Interestingly enough, the finding reveals a close interrelationship between workplace and personal lives for staff in terms of gender equality. In this sense, work and family divides were bridged in the way that staff considered gender issues into their homes. Significantly, they adjusted their attitudes towards gender roles and norms at home on the basis of the gender awareness, knowledge, and perceptions they gathered at work. It is worth noting that male staff reflected this impact more realistically through their recognition of equal contributions between men and women in all aspects of marriage. Although this positive impact was widely realized by all respondents, it was potentially upsetting to other goals to revert to the difficulty in employing more men in the INGO as a common obstacle facing the development sector in Vietnam. Nonetheless, it is crucial to note that the institutional investment in building the capacity of staff in this area is having a wider and more personal and societal transformation in employees' families and homes.

\subsection{Conclusion}

In short, the INGO has built the initial bricks of a foundation to mainstream gender into its organizational policies and programs and/or projects. Being aware of gender work as a timeand resource-consuming process, the organization has made a remarkable investment in making this process work technically. As a crucial component of gender mainstreaming efforts, the organization set working directions, strategies, and policies to implement it as effectively as possible. The unanimous determination of all people involved brought about some considerable achievements in progress towards pushing gender to the centre of their 
development work. However, some of key challenges facing these efforts are uncovered. They were the conceptual obscurity, the lack of human resources, the insufficient budget, the limited technical capacity of staff, the absence of M\&E and Accountability mechanisms, and the inadequate attention to gender based organizational culture. Meanwhile, the findings showed some opportunities for the organization's contribution to addressing gender issues in Vietnam. They included the potential to make change in wider society through improving staff's perceptions and attitudes towards gender roles in their professional and private relationships, the contribution to building capacity of gender analysis and planning for local agencies, and strengthening the dialogue on gender work amongst NGOs. 


\section{Chapter 6: Findings and Data analysis in the LNGO}

\subsection{Introduction}

This chapter addresses the research question through a case study of an LNGO. Findings are mainly from semi-structured interviews with two key staff in the organization. They are quoted as: (female) Deputy Director (LNGO 1.1) and (male) Project staff (LNGO 1.2). This part provides critical insights into the extent to which gender has been integrated in the organization itself and at project level. It comprises two main sections. The first section is a brief about the organization and its current working mechanisms. The second section presents primary findings from in-depth interviews and the discussion on findings.

\subsection{Overview of the LNGO}

This is a Vietnamese non-governmental organization, founded in 2008. Previously, it used to be a Health Consultancy and Investment Company, which worked in the areas of health, gender, women's empowerment, reproductive health, sexuality, and HIV \& AIDS. This LNGO was established in response to the complex issues of health and population that had arisen in contemporary Vietnamese society. The organization expected to contribute to addressing these issues through its creative initiatives, strategies and approaches. Its vision was to strive for recognition, locally and internationally, as a pioneering organization providing development activities following creative models of intervention which aimed to realistically meet social demands and provide the best consulting services in the areas of health, social sciences and environmental protection. The LNGO was mandated to promote equality, diversity, and good health for all through a multi-sector-based approach to research, policy lobbying, and intervention in communities. The LNGO's activities were grounded in all participatory, rights, capacity development, social justice and environmental friendly-based principles. Its four main activities are: promoting research, enhancing capacity, developing creative models of intervention, and sharing research results and policy lobbying (Doc 1).

The organization carries out research to identify and reflect crucial factors affecting Vietnamese people's health and lives. The coverage of its research extends to health policies and assessing new models of healthcare. Its qualitative and quantitative research was 
targeted at initiatives and methods creative and specific to real contexts and gender sensitivity. The research prioritized issues of subordinate and minority groups such as female victims of gender-based violence, people living with the HIV-infected, and the disabled. One of the important principles in the LNGO was to build capacity for partners. The organization achieved this goal through assisting partners to develop capacity development strategies, training, monitoring, and workshops. The LNGO developed training programmes and knowledge updates under the themes of gender and gender integration, sexually and sexual health, rights and rights-based approach, reproductive health and HIV, public health, environmental health, project planning, monitoring and evaluation. The organization was a pioneer in piloting models using a multispectral approach in a creative and innovative manner. These models were designed to ensure pro-active participation of individuals and the community in improving status, health and living quality. The LNGO believed that its research results needed to benefit the research's participants, local partners and policy makers. The organization conducted policy lobbying in local and international workshops, forums in mass media and networks with other organizations. The LNGO was in partnership with local bodies such as the Ministry of Health, General Statistics Office, Vietnamese Women's Union; universities such as the Universities of Latrobe, Monash, and Melbourne; development institutions such as UNIFEM (now UN Women), UNAIDS; INGOs, such as PACT, Pathfinder International, and FHI amongst many others. Moreover, the LNGO was an active member of LNGO's network, Dovipnet (Domestic Violence Prevention Network in Vietnam) and others (Doc 1). The organization was recognized to be strong in terms of numbers of staff, activities, donors, partnerships and the financial capacity in the network of LNGOs (LNGO 1.1).

\subsection{Findings and Data analysis}

\subsubsection{Institutionalizing gender in the organizational policies}

The findings illustrate how gender mainstreaming was implemented in the LNGO's office and technical projects. In the staffing policies, gender mainstreaming was interpreted as the creation of favourable working conditions for both sexes. Women's and men's issues were carefully analysed and taken into consideration when designing staffing policies. The organization was thought to be a comfortable workplace in which female and male staff at 
all levels were free and equal to speak their thoughts in all aspects. The two main issues, nonetheless, that the LNGO has faced and continued to face, were high staff turnover and unbalanced gender ratio (more female than male staff). Some of the reasons for these two issues had to do with traditional gender roles and gender stereotypes in Vietnam.

\section{a) Gender in staffing structure}

In this organization, there was an unequal ratio of men and women in the staffing structure; as LNGO 1.1 mentioned, there were two male staff while the rest were women. This informant, however, noted that the management level included two men and two women in which the Director of the LNGO was female. The lack of balance between the numbers of male and female staff led the organization to attempt to employ more men. Although the recruitment advertisement did not encourage men specifically to apply, the idea of "prioritization" or "paying attention to find male applicants" was demonstrated in the round of screening Curriculum Vitae (LNGO 1.1).

Given that the number of female applicants was always higher than that of men, it became very difficult for the organization to recruit more male staff. LNGO 1.1 admitted that this reality was common in the development sector while, to her knowledge, addressing the gender issue needed the involvement of both men's and women's efforts. More than that, LNGOs in general had to deal with challenges in retaining staff because of high staff turnover (LNGO 1.1). LNGO 1.1, as a Deputy of the organization, offered some reasons as follows. Firstly, the nature of development work required much travelling: being absent from home prevented women - particular those with children and family - from fulfilling the traditional role of Vietnamese women. As a result, they may choose to shift to different sectors with fewer or no business trips (LNGO 1.1). Retaining male staff in the organization was even harder. After accumulating experience, they often moved to an INGO for better remuneration. In terms of salary, LNGO 1.1 did not deny its inability to pay the staff as much as its international counterparts could offer, while cultural and traditional norms in Vietnam pressures men to act as the family's breadwinners. LNGO 1.2 also speculated that Vietnamese men had a tendency to gather with other male colleagues to play games, or drink tea or beer, or share other so-called men's common interests after working hours 
(LNGO 1.2). This represents the typical culture of workplaces in Vietnam. Understandably, men felt bored in a workplace with a majority of women, as LNGO 1.2 noted.

\section{b) Staffing policies for men and women}

The personnel policies of the LNGO were said by LNGO 1.1 to be mainly in line with those in the Labour Law issued by the Vietnamese government. In addition, the organization had some separate privileges tailored for female and male staff with an aim of facilitating their best work performance (LNGO 1.1). According to LNGO 1.1, particular challenges for female staff working in development work have been considered, in order to design specific support. For example, working time for pregnant staff was very flexible to accommodate their regular health checks (LNGO 1.1). Similarly, male staff were allowed short breaks whenever their wives gave birth (LNGO 1.1). The LNGO could bear a part of the total cost if women travelling on business wished to bring their children and a companion along. This support was based on the thoughtful consideration of leaders, including LNGO 1.1 that a pregnant woman or one with a new-born child may lose her opportunity to network with people. Although travelling was not mandatory for female staff, they were encouraged to do so with some financial support from the organization (LNGO 1.1). LNGO 1.2 as one of two men in the office voiced his strong support for this policy arguing that travelling too much would affect women's physical health. Opportunities for job promotion, career development and studying were equally shared by female and male staff as said by all respondents.

\subsubsection{Organizational culture}

The organization was staffed by all local people and led by highly academic and strong women. LNGO 1.1 - the Deputy Director - viewed herself as " $a$ strong and advanced woman". Despite women surpassing men in the office, there was no power bias in decisionmaking process at all levels. LNGO 1.2 felt comfortable working in a place where everyone was free to speak their minds. According to LNGO 1.1, all members of the Board of Managers had an equal voice and vote in any discussion or decision. Male deputies did not feel subordination or pressure under the leadership of a woman (LNGO 1.1) while male staff were not marginalized or discriminated in any activities of the organization. 
Considering the majority of women in the office, the LNGO proved to be prompt in bringing women's burdens and gender into the centre of its casual talks and events. LNGO 1.1 thought it could be easier for a small-sized organization like the LNGO to change attitudes and perceptions than for an agency of 50 staff or more. LNGO 1.2 - a male staff always felt excited to join the casual talk about this theme during the lunch time. Furthermore, retreats or events on some special occasions were organized with the participation of staff and their partners and children, at which gender made for one of the topics of conversation (LNGO 1.1). LNGO 1.1 reported that the attendants of these activities were staff and their spouses and partners. LNGO 1.1 considered it a good chance to improve people's awareness of gender issues while staff's partners got to understand more about the nature and requirement of development work. The outcome she expected was deep sympathy and support paid by husbands and wives to their partners who were working for the LNGO. LNGO 1.1 pointed to out her husband's awareness and knowledge of gender issues changed as a positive impact of these events after he had joined her in these activities.

\subsubsection{Staff capacity of gender}

\section{a) Multi-functional staff}

In saying "Multi-functional staff" or "All-in-one staff", LNGO 1.1 meant that one staff had to take on different roles at the same time. In other words, this member of staff could undertake various tasks from $A$ to $Z$ as required. The organization's tendency was to train staff to be "multifunctional" (LNGO 1.1) (see Figure 6.1). 


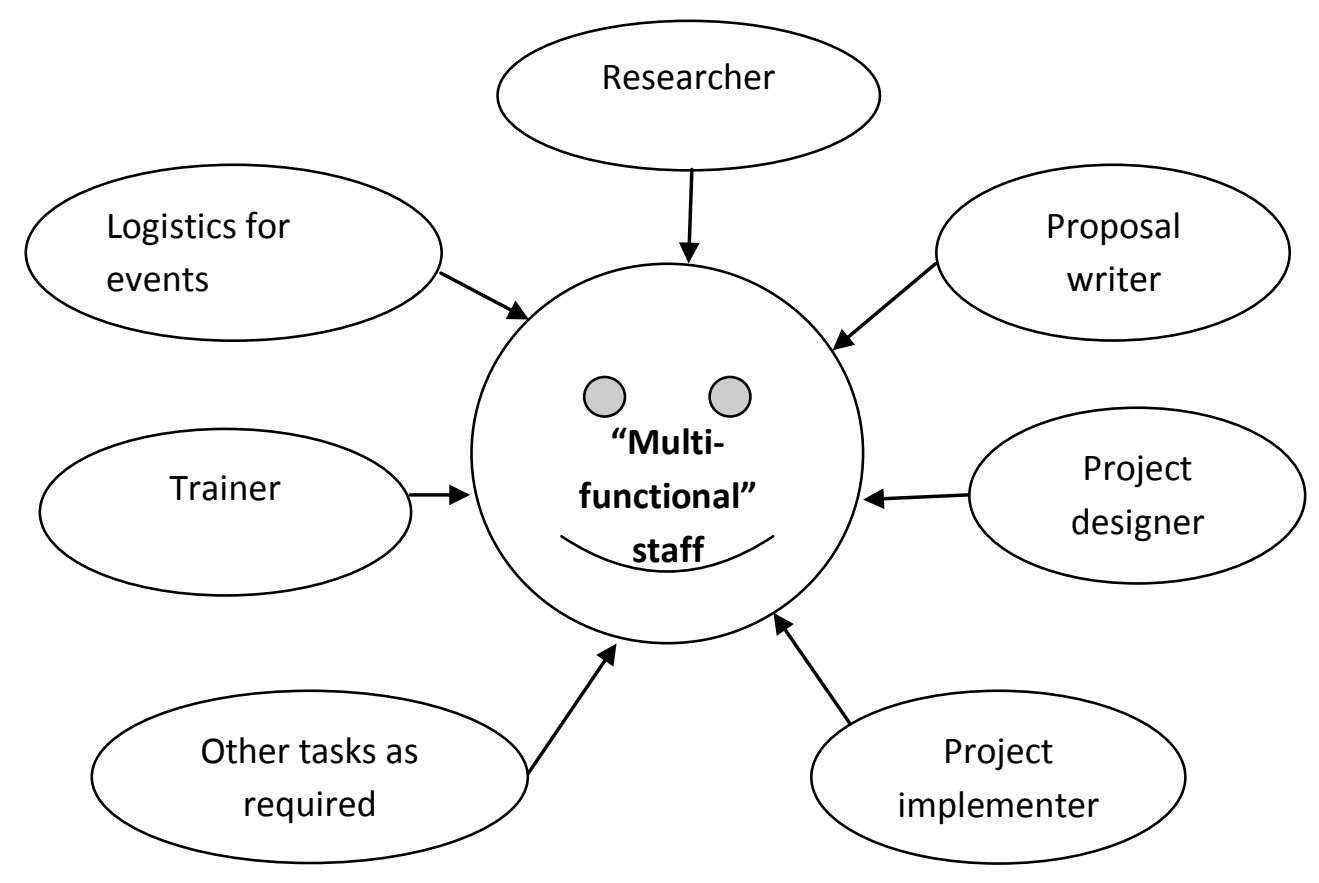

\section{Figure 6.1. Illustration of the 'multifunctional' staff model in the LNGO}

According to LNGO 1.1, a staff was in charge of writing proposal, designing, implementing a specific project, being trainer, researcher, and organizing logistics for workshops. It was believed by LNGO 1.1 that this would be a good way to integrate gender into each element of each employee's work. According to LNGO 1.1, three members of the Board of Managers were in charge of different areas of activities in the organization while specializing in gender. LNGO 1.1, as a Deputy, was in charge of managing human resources while taking a lead in the area of domestic violence programmes and projects. Given that, gender concerns were considered by one person throughout the whole process from designing to implementing, in all activities, training kits, and in working with local partners (LNGO 1.1). LNGO 1.1 considered this a way of ensuring the consistency in gender integration because gender work, from designing to implementing a project, was done by just one person.

\section{b) "Daily coaching" - an approach to gender trainings}

Starting from the idea of developing a "multifunctional" team, the LNGO chose to provide daily coaching of gender integration to staff. That was the key to equipping staff with essential awareness, knowledge and skill of carrying out gender work (LNGO 1.1). Perhaps the small size of the LNGO in terms of staff numbers enabled these leaders to provide on- 
the-job training for staff on how to sensitize gender in all they were doing. LNGO 1.1 presented an instance of a domestic violence project in which she asked her staff to work with not only Women's Union, but also the local People's Committee and Police Agency (LNGO 1.1). In doing this, LNGO 1.1 aimed to steer employees to think of violence not as a women's issue, but of the whole of society. In reality, the organization did not have a GFP unit or person. The point of daily coaching was that staff had a better understanding of gender when it was put in the specific contexts or activities of a project (LNGO 1.1 and LNGO 1.2). LNGO 1.2 explained that he had improved gender sensitivity given the opportunity to look into gender issues in detail. Staff were thus given a chance to internalize gender in a way that moved it beyond a matter to be understood as a theoretical construct (LNGO 1.1).

\subsubsection{The importance of including men in gender work}

LNGO 1.1 understood gender as the equal concern for both men and women. In other words, it would not be over-privileging for women and under-privileging for men. Looking from a gender perspective, both sexes should have equal access, control and benefits from development, as LNGO 1.1 mentioned. To some extent, focusing on women in isolation would disadvantage men, given the reality that Vietnamese men also had certain sufferings caused by the traditional gender norms (LNGO 1.1). In LNGO 1.1's thought, "doing gender is not necessarily always standing by women and blaming men" because doing so would prevent men's cooperation in addressing gender issues. She pointed out some Vietnamese men's difficulties, for instance, there being "a lot of clinics for obstetrics and gynaecology while just a few service providers for men" - and added that Vietnamese men grew up with pressure to earn enough money to support the whole family. This gender prejudice was more obvious and pervasive in rural areas and cause of gender-based violence as LNGO 1.1 indicated. From this circumstance, a male staff member could be more convincing to men in communities than could a female staff member because two men could talk "as friends" (LNGO 1.2).

\subsection{5 “Internalizing" gender into daily work and life}

The terminology of "internalizing" gender was repeated by LNGO 1.1 through the whole talk. 'Internalizing' was defined as "making gender issues your own", so that people could think of 
and see gender in everything they did (LNGO 1.1). LNGO 1.1 believed gender work would be more effective if people made sincere efforts to change the status quo and felt "more or less the necessity for this change to happen" (LNGO 1.1). In that sense, LNGO 1.1 noticed that gender would never be internalized if people continued to consider gender work as the duty or responsibility of a focal point person only. LNGO 1.1 stressed bringing gender into staff consciousness in the whole process, including project activities or working styles. Above all, the intention was that staff could see the benefit of their work to their private lives or relationships with husbands and partners (LNGO 1.1). Eventually, internalizing gender did not only help staff to work better in project sites but also analyse their personal relationships using gender lenses (LNGO 1.1). The change extended to people rethinking their attitudes and behaviours towards gender roles at home.

\subsubsection{The absence of institutional documents of gender}

The definition of "internalizing" and the negotiation process of including gender in the daily work and private lives of staff came as an interest partly because it derived from the thought of actively using everybody to take action, rather than just providing them with training or employing a GFP person. That was LNGO 1.1's viewpoint as a Deputy Director of the organization. However, it was "easier said than done" because there were no gender mainstreaming strategies, policies and guideline kits commonly used by the whole organization. In spite of being aware of the need for institutional documents, no timeframe for drafting and issuing them has been set to date due to time constraints and the financial burden of maintaining the office. Managers and Deputies noticed the essential importance

of drafting a standard document of gender integration, yet their tight work schedules caused them to lag behind on this task. LNGO 1.1 admitted that, "I can only work on this when I am free from other tasks". More likely, the task suffers in the competition with other performances in the LNGO. In addition, staff needed to be convinced of the intimate relation between gender work and organizational benefits in the long-term so as to have common and appropriate understanding of gender work (LNGO 1.1). She recognized the reality that persuasion was difficult given the staff were not a homogeneous group (LNGO 1.1). The plan for gender institutionalization was often delayed, with the explanation for this from LNGO 1.1 that the organization had to deal with other concerns of the LNGO including inputs for 
projects, fund-raising, and expenses for the daily functioning of the office which were more important and immediately responsive.

\subsubsection{Operations under donor-dependent regime}

Financially, all operations of the LNGO were found to be dependent on donors. The LNGO is a self-established, self-sustaining and self-maintaining organization, rather than a sub set of a head organization. Therefore, raising funds to maintain their office and operations became "the biggest headache issue" for the organization (LNGO 1.1). Comparatively, LNGO 1.1 said this was the most notable difference between a local NGO and an International NGO. LNGO 1.1 was honest about the impossibility for the LNGO to make annual organizational plans at the moment because no donor had made a fixed financial commitment. Hence, the LNGO's current operations were very much piecemeal because "fund allocation is not secured" (LNGO 1.1). This resulted in the dispersion of the LNGO's interventions, as LNGO 1.1 described:

"... we always have to switch from this project site to the other and carry out a lot of different activities at the same time to attract more funding. For instance, if the LNGO's proposal was found to be compatible with a specific donor's strategies, this donor might give you funds to implement it. However, it does not mean that this donor will secure the continuation of its funding in that area to seek for significant and deep impact once finished" (LNGO 1.1).

Consequently, the LNGO had no choice other than to embark on projects in other areas and/ or other sites in order to receive funds to both run the office and continue interventions. Eventually the LNGO's interventions are diluted.

\subsubsection{Gender impact in communities}

The above sections reveal the typical challenges which impede the LNGO from making notable gender impacts in the office and communities noticeable. In the organization, there is a high level of appreciation for utilising daily coaching instead of hiring a GFP unit or person and internalizing gender into daily work and personal relationships. Speaking personally, LNGO 1.2 self-evaluated his improvement in gender sensitivity and advancement 
in his thinking about gender roles, which were deeply rooted in Vietnamese cultural and traditional norms. This seemed a positive and encouraging effect of working for the LNGO in that he started to think about the equality between him and his future wife in decisionmaking power at home. Imposition in family had not been his intention although he grew up with a father who had always taught him to view women as junior and subordinate (LNGO 1.2).

There was no monitoring and evaluation system found in the organization. However, LNGO 1.1 summarized the alternative process as follows. Some programmes of the LNGO carried out research on the impacts generated by the organization's interventions. This research was participated in by the direct beneficiaries of the programme or project, or officers from departments, and aimed to see the change in attitudes towards, and perceptions of, gender issues. The cross-checking between participants was designed in the form of a mini-survey. The task-based questions were used to measure knowledge, attitudes and reactions to specific circumstances. Nevertheless, not all programmes could afford to conduct this kind of research (LNGO 1.1). Considering the LNGO's projects were almost all short-term, the realizable impact was to make people understand the inevitable involvement of men in addressing gender-related issues, such as domestic violence and sexuality, which used to be viewed as women's issues by the local people (LNGO 1.1). LNGO 1.2 spoke of the impact in communities where he used to work:

“... significantly, local men in project sites showed their readiness and comfort to engage in activities, so that their knowledge of gender issues was changed after the intervention. And, trainers are asked to keep their eyes on women and encourage them to speak. Women are prioritized to answer before men".

\subsection{Conclusion}

Much of the work in gender integration in the LNGO was done in actions, rather than in institutional papers. The findings revealed the unavailability of technical frameworks, checklists, and tools for gender integration in policies and programmes. In addition, the LNGO did not provide training for staff in gender awareness and planning, monitoring and evaluation. Alternatively, the organization domesticated the process of staff training to fit its 
exigencies on the basis of its available human and financial resources by taking up three approaches: "multi-functional staff", "internalizing gender", and "daily coaching gender". All three techniques aimed to sensitize staff to gender and make them passionate about gender equality in both their professional work and personal lives as summarized in the below Figure6.2:

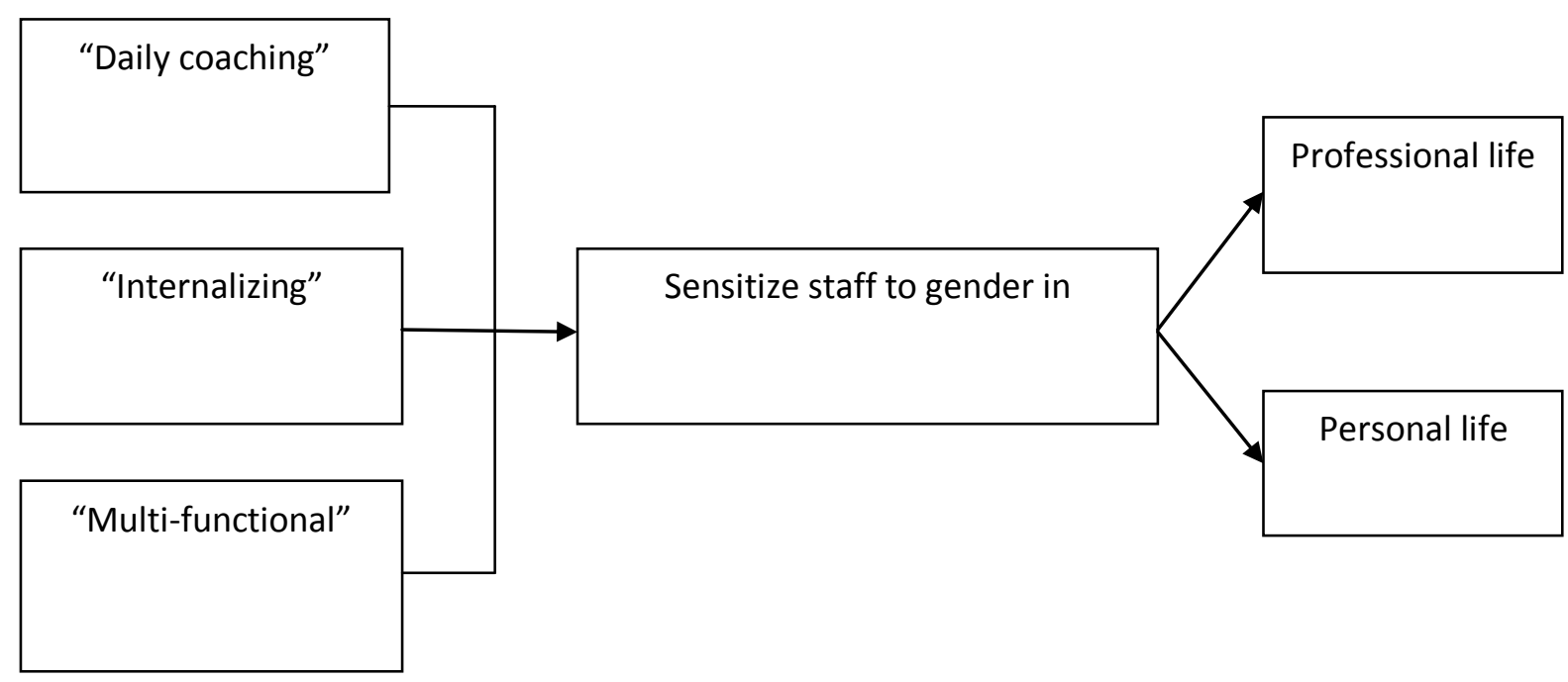

\section{Figure 6.2. Summary of strategies used to achieve double-tier goals in the LNGO}

These techniques worked to move gender work into the centre of individual staff consciousness, and the organization's work. In practice, however, gender remained at the margin of the LNGO's operations mainly through other obstacles. These obstacles were time-constraints on staff, a donor-dependent paradigm, and no institutional consistency. These factors hindered gender mainstreaming, so that gender work was side-lined in many cases. As a result, these techniques appeared to have little potential for integrating gender systematically and consistently into office procedures and in communities to bring about gender equality and justice.

The findings also drew on: the recognition of the importance of men's involvement in addressing gender inequality and inequity in both office and communities; the close interaction between leaders and staff; and formulation of the link between workplace and home in terms of gender issues and gender relations. The LNGO strived to create interrelations between work and home with the aim of raising gender awareness for employee's 
spouses, and setting female staff free from persistent pressures caused by gender bias and stereotypes. 


\section{Chapter 7: Discussion, Recommendations, and Conclusion}

\subsection{Introduction}

Gender Mainstreaming, in definition, was thought to be the ideal strategy to address gender inequality. Once launched in 1995, it was widely adopted by governments, development agencies, and other stakeholders. A decade later, a wave of sceptics about its effectiveness emerged in the development sector. With this starting point, the central aim of my thesis was to investigate how NGOs based in Vietnam applied this strategy in practice in the specific context of Vietnam. The study hoped to see whether such NGOs fell into the same situation as that found in the development mainstream.

In Vietnam, where gender bias and stereotypes were largely influenced by Confucius doctrine, looking at gender integration in NGOs' operations and activities appeared crucial. The effectiveness of NGOs' work could potentially make significant impacts in terms of challenging cultural and social expectations about how men and women should act in society. As discussed in Chapter 3's discussion of Background and Context, although gender issues have drawn much attention from the government and other stakeholders, gender inequality has been improved only to a modest level due to the inconsistent enforcement of relevant laws and the implementation of superficial interventions. Considering how the gender relationship has become more complex as a cause of economic growth and development in Vietnam, it was necessary to appeal for increased efforts from various stakeholders including NGOs, which were invariably imbued with a sense of intrinsic interventions. Eventually, real impacts on gender relationships could be generated.

My research was built on gender issues in Vietnam and attempts to address them in the context of evolving critiques over the application of gender mainstreaming in practice. The main claims were that gender mainstreaming ended up being ineffective and inefficient in practice. I explored this through the review of gender mainstreaming in different NGO contexts. This allowed me to draw on reasons for the failure of gender mainstreaming in those contexts and explore if there were alternative ways to make it better work in reality. In the remainder of this final chapter, I discuss the findings of four research sub-questions to answer the central research objectives illustrated at the beginning of this chapter. Following 
the discussion, I offer some thoughts for areas of future research and then concluding remarks.

\subsection{Discussion}

\subsubsection{Where are NGOs in the process of gender mainstreaming?}

The first two questions I explored were: to what extent has gender been mainstreamed, firstly, within the organization, and secondly, in their projects. These questions contributed to the main objective of elaborating on how both INGO and LNGO institutionalized and implemented gender mainstreaming in their policies and activities. Within the limitations of the findings, it was hard to be conclusive as to whether the organizations had failed or succeeded in doing gender work at this point. Nevertheless, it was obvious that both organizations had begun to accept gender mainstreaming as a good idea, had had it on board since 2008, and were gradually starting to integrate it into structures and practice. Like many examples in the international literature, however, such progress in both the INGO and the LNGO was not clear-cut or linear(Macdonald, Sprenger, \& Dubel, 1997).

In the literature review, it was posited that gender mainstreaming happened in an organization first (C. Moser, 1993). This could be achieved through the changed gender norms, attitudes and practices of staff who incorporated gender concerns in all policies, strategies, and procedures of their development work. Oxfam, for example, required all staff to have a sound working grasp of gender analysis. According to Wallace \&Wendoh (2005), this foundation helped sensitize staff to gender in their work. Organizations in Malawi mentioned in Tiessen's article (2007) started their gender mainstreaming by employing more female staff in the office. The two organizations in this research adopted different approaches to gender work, based on their own organizational conditions and characteristics.

The literature review pointed out a common tendency in many international development institutions which is to begin gender mainstreaming with technical aspects(Mukhopadhyay, 2004; Standing, 2004). The findings in this study revealed that the INGO was not an exception to that trend. As such, the organization assigned a GFP person to facilitate and 
coordinate the whole process. The INGO also had Gender Taskforce with decentralized focal points facilitating the operationalization and implementation of gender mainstreaming into the specific activities of the organization(Macdonald et al., 1997). As well, the INGO developed the gender mainstreaming strategy, provided staff with gender training, formulated staffing policies, and allocated funding. This approach had strong backing from the top leaders and goodwill from the staff. These factors enabled staff to raise their awareness, knowledge, and capacity associated with gender mainstreaming but were not enough to make all staff sensitive to gender as Mitchell (2004)also discussed in the case of the United Nations (UN). The findings unveiled some key challenges facing the INGO in its gender mainstreaming. They were:

(1) Lack of conceptual clarity;

(2) Infrequency and no in-depth of trainings;

(3) Limited technical capacity and skills of all staff at all levels;

(4) High staff turnover, lack of human resources, and an unbalanced ratio between men and women in staffing structure;

(5) Insufficient budget;

(6) Malfunctioning of Gender Taskforce;

(7) Lack of institutional frameworks;

(8) Absence of M\&E and Accountability mechanisms;

(9) Lack of attention to the importance of organizational culture in challenging gender norms in wider society

These obstacles came as no great surprise because they were in common with those presented by key academics, practitioners and researchers such as Mitchell (2004), Reid (2004), Standing (2004), Kabeer(2005), C.Moser(2005), A. Moser \&C. Moser (2005), A. Moser (2007), Tiessen(2007), and Subrahmanian(2009) amongst others. These authors concluded that the technical tools associated with gender mainstreaming were not enough to allow gender transformation of male-dominated cultures. As a result, there remain gaps between rhetoric and practice. 
The authors cited above also argued that technical tools need to go hand in hand with a political will, but that this has been lacking in the current development mainstream (Corner, 2004). According to the findings of the INGO case study in Vietnam, despite strong backing from the top leaders, the gaps between stated aims and affirmative action were still large because no official commitment had been made to gender work in terms of funding and human resources. Despite goodwill from staff, gender had not become a natural component of their work due to the inconsistent mechanisms and irregular provision of gender training. Also, GFP staff seemed to have no power to challenge the leadership. In addition, a large organization like the INGO housed different views on the importance of gender equality and different interpretations of its concepts between departments. They, therefore, took on gender issues at a different pace across the organization (see also Macdonald, et al., 1997).

On the other hand, the LNGO began its gender focus with the personal, rather than the technical, through three techniques of "multi-functional staff", "daily coaching", and "internalizing gender". While encouraging, the unavailability of a GFP, technical policies, strategies, frameworks, and toolkits for gender integration in the LNGO also limited gender mainstreaming outcomes reflecting what was identified as conceptual obscurity in international literature. O'Neil (2004) took the issue of conceptual(language) obscurity as an explanation for the misunderstanding gender mainstreaming in mainstream development. Mitchell (2004) expressed serious concern over the implementation of gender mainstreaming if people remained unclear about how it is defined in practice.

To avoid the complexity of this concept itself, the LNGO used the term "gender internalization". This was domesticated through the organization's key goal of narrowing work and home divides in terms of gender. Hence, the staff were benefitting in their personal lives as a result of their gender work in the NGO. It enabled them to improve gender relationships in their families and in wider society. The LNGO emphasized developing passion for gender change in the staff's professional and private lives. Once aware of the important effect of their work in their private lives, it was thought that all staff could make concerted efforts to sensitize to, respond to, and transform gender inequalities in their professional work. The LNGO chose this strategy to make gender work very 'real' and 'personal'. Given the organization's small size and relatively simple staffing hierarchy, the 
close relationship between leaders and staff and amongst colleagues, needed for this technique to work, was easily established. This closeness played an important role in facilitating the daily coaching approach aimed at internalizing gender in people's minds and hearts. Accordingly, the idea was that theywould be able to view everything through a gender lens.

\subsubsection{The unclear boundary between WID and GAD approach}

The findings demonstrated the continued prevalence of the WID, rather than the GAD approach in both organizations' operations. Although the term 'gender' has been adopted by both since 2008 , there was on-going confusion between WID and GAD. In line with Mukhopadhyay (2004) and Rao\& Kelleher (2005), this misunderstanding led to an overemphasis on women's issues and an under-estimation of men's importance in addressing gender issues. As a result, in both the INGO and the LNGO, female staff were entitled with some privileges which helped them to better fulfil their respective roles at work and at home. All respondents believed that this was positive for female staff. As such, INGO 1.2 the Human Resource Manager of the INGO - could not think of what more the organization could provide for male and female staff in relation to gender equality. INGO 1.2 reflected that the gender mainstreaming in human policies appeared far more complex than what she had learnt from densely drawn abstract materials and training. All incentives given by the organization were visible and recognizable; however, the incentives did not ultimately do much to emancipate women from their more traditional gender roles.

Nevertheless, gaining more confidence and empowerment in public did not always lead to women enjoying equal relationships in the domestic sphere. In other words, both NGOs did not analyse the changes in women's private lives within their relationships with husbands and their communities owing to their increased confidence and power in public. According to the findings, there was a discrepancy between the behaviour of women in public and private. In the INGO, female staff were provided with awareness, knowledge and training on gender to work with communities. All informants expressed their happiness and comfort at working in these two workplaces where female and male staff were entitled to equal opportunity and the chance to be heard. However, as Mukhopadhyay (2004) argued, such 
outcomes cannot be the ultimate goal of gender mainstreaming. For example, when invited to talk about bringing gender back into the home, female informant INGO 1.4 thought,

"... back home, it is very difficult to change. There is no equal relationship between husband and wife. For example, if a guest comes to visit and catches my husband washing dishes, the immediate reaction the guest may have is my husband's face is lost by being junior to wife."

In the INGO, people continued to consider that women-targeted projects empowered women better than gender projects. Certainly, women-specific interventions were believed, in the literature review, to remain crucial for the sake of gender equality. Similarly, women in communities were reported to gain more confidence and involvement in decision-making and empowerment in public thanks to project interventions. However, the traditional behaviour vis-a-vis husbands or partners and children in their domestic roles remained persistent because "a female chairman of the local Women's Union is not permitted to attend her family's meetings" (a story told by INGO 1.5).

\subsubsection{Taking men's issues and men's involvement on board to address gender inequality}

There is a recently evolved need to consider both men's and women's gender issues and such action is the responsibility of both women and men. In reality, men may also face gender issues in their professional and private lives which might affect their work performance. However, it is quite easy for people to disregard men's gender issues with the explanation that they are viewed as obstacles to women's empowerment (Flood, 2004). In this sense, the LNGO was found to have a clear opinion and strong action on involving men in the whole process. In the first place, the LNGO sought the sympathy of husbands and partners for female staff in office. Traditionally, men played a crucial role as decision-makers and community leaders and there was an intimate link between gender inequality and men's practices and identities. In communities, male beneficiaries found it comfortable talking to male gender staff. In other words, a man was often in a good position to influence other men, regardless of colleagues or partners or beneficiaries, as men listened to men more readily than they listened to women. In this case, women may have real difficulty in making 
their voices heard. The findings pointed out the contrary situation for a woman who worked on gender issues; she might be viewed as a protester, a revolutionary, or a woman who has excluded herself from her culture (INGO 1.2). In short, gender concerns can become more normal, more pragmatic, and more integral to the development process through the engagement of men(Cleaver, 2002).

Also, there were examples of men working on gender in both organizations who did not have to deal with instances of institutional resistance within his own organization but some personal and family issues. In both organizations, male gender staff were likely to be at a disadvantage compared with women. INGO 1.1 shared his trouble that he was thrown 'questioning' eyes from a meeting with people from outside the organization; he felt they thought he lacked a strongly masculine identity, given his work on gender issues (Macdonald et al., 1997) which were traditionally supposed of and for women. The male respondent in the LNGO had to deal with the same problem when his male friends commented that women were in the majority at his work place. Some thought NGO's working environment was not suitable for men considering that, in Vietnam, alcohol and peer groups made an element in men's typical work place environments, while some mused "lucky you to be working with a garden of beautiful flowers" (LNGO 1.2). This was offered as one reason for men to be missing in the development sector in Vietnam: men needed an enabling environment provided by gender identity(Doyle, 2002).

It is perhaps worth noting the positive that those men working in NGOs felt unaffected by the resistance and misconception of people towards their work. Yet both organizations were challenged by the difficulty in attracting more male staff to the development sector in general, and towards working in the gender area in particular. In the present circumstances, the INGO and the LNGO were still trapped in the dilemma of the need for male staff and the inability to recruit them. In office, a popular reason found in the LNGO was that male staff left development jobs for higher paid jobs in different sectors, mostly business and trading. In communities, the inability to be a main money maker may lead men to considerable insecurity and frustration, leading to violence (LNGO 1.1). The masculine culture of Vietnam expected men to fulfil specific roles as husbands and fathers. These expectations became heavy responsibilities on men's shoulders. 
Having more men in the sector become a more inspiring indication when both organizations' male staff showed improved attitudes and behaviours towards gender roles. It seemed that men brought gender back home and started to share domestic responsibilities with wives. The cases of men working for the INGO served as a good reflection of this change. Men changed how they viewed women. For instance, LNGO 1.2 (male) was taught as a child about the subordinate position of women in families and society but had more or less changed his perception and consciousness of gender. Nonetheless, his change went hand in hand with traditional gender hierarchies. Men - particularly young men - are accepting change but at the same time hanging on to traditional views. Talking behind the interview, LNGO 1.2 admitted frankly that, the more educated a girl was, the harder she would be chosen by a local man. Overall, the positive change in male development workers' attitudes should be acknowledged, and thus it is an appropriate time to include and engage men in addressing the deep-rooted social constructs of gender inequity that affected both women and men(Bannon \& C.Correia, 2006).

Changing men's behaviour is crucial to creating the social space where individual women and men can negotiate identity and offer hope for broader social change. To achieve that, interventions will have to move away from simplistic intentions to more complex ones that see men as gendered beings in order to involve men (Doyle, 2002). Besides attempts to increase the visibility of women's equity and empowerment in office and in communities, the LNGO started to realize the essentiality of considering gender matters for boys and men and recognizing the costs or stresses of male socialization and identities on men, their families, and society as a whole. There are very few clinics for men's sexual issues in Vietnam (LNGO 1.1). Regarding gender-based violence for instance, interventions directed at women are often ineffective or unsustainable if men do not cooperate or are not involved (Bannon \& C.Correia, 2006). Hence, respondents in the LNGO posed the need for understanding about gendered identities and pressures, and that these, in turn, are linked to some of the most problematic and intractable development issues of the day, such as healthcare, domestic violence, and HIV/AIDS (Bannon \& C.Correia, 2006). 


\subsubsection{NGOs: An organizational culture makes positive gender impacts in wider society?!}

The third and the last question I explored was: what opportunities for NGOs' gender work to contribute to addressing gender inequality in Vietnam, and how is gender considered in the organizational culture in these INGOs? These, along with the findings of two previous questions, allowed me to achieve the objectives of firstly providing critical insights into the opportunities these organizations present in their efforts to mainstream gender, and secondly, exploring the significance of these NGOs' organizational cultures to gender transformation in a wider society. The boldest point in NGOs' gender work was in the valuable impacts they had on personal and societal transformations in employees' families and homes. This was a result of building gender capacity and organizational culture for their staff. Rao and Kelleher (2005) drew on the informal cultural norms and exclusionary practices as an integral contribution to make gender mainstreaming policies work as in theory. Rao and Kelleher (2005) continued to name cultural systems together with political access, accountability systems, and cognitive systems - four inter-related factors for organizational change in gender (see Figure 7.1). 


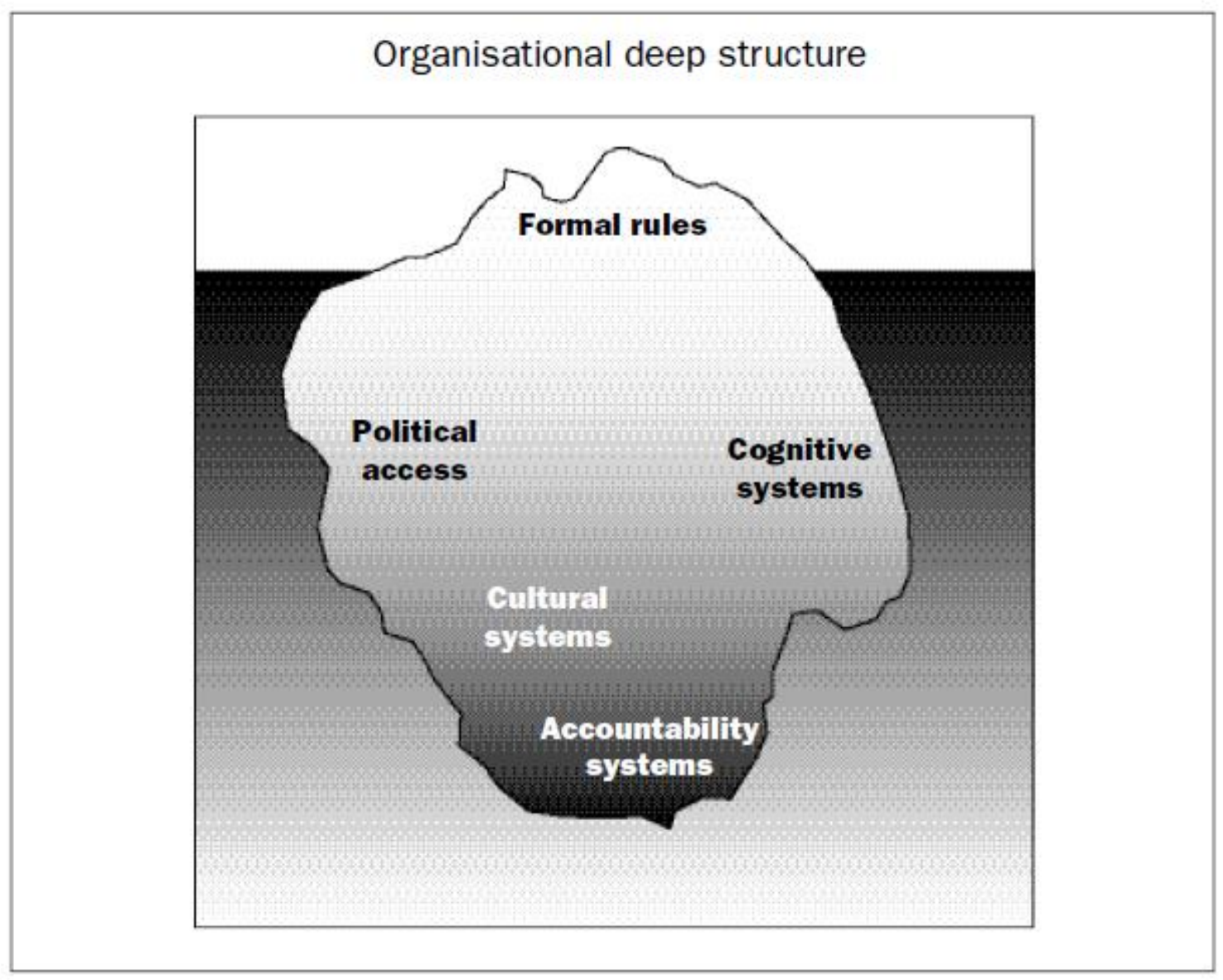

Figure 7.1. The iceberg of organizational structure(Rao \& Kelleher, 2005, p. 65)

One aspect of the deep structure is the work/family divide perpetuated by most organizations which prevents women from being full participants at work, as women continue to bear the responsibility for the care of children and old people in their family(Rao \& Kelleher, 2005).

Women were less likely to apply for posts in rural projects as found in both organizations because their jobs require them to extensively travel in remote areas. This became a constraint on women's mobility at work as they are mainly responsible for domestic affairs, and thus expected to fulfil their responsibilities at home. As a result, they found it difficult spending extensive periods away from home. It is a hard situation because rural development initiatives are focus areas for NGOs. The majority of female applicants for rural posts are single. Those who are married with children find it hard for their career path in the 
development field in general. This point was a part of Tadele's(2001) findings in the context of Ethiopia and Rea (2004) in the Australia workforce.

Rao and Kelleher (2005) defined organizational culture as a "collection of values, history, and ways of doing things that form the unstated rules of the game in an organization" (p.66).

This sphere has the capacity to make things happen or block them. In some cases, culture can exclude gender to focus on more 'real' outcomes for the organization. Organizational culture was one of five spheres from which, Rao and Kelleher (2004) believed, power can be generated to have organizational transformation, as can be seen in Figure 7.2:

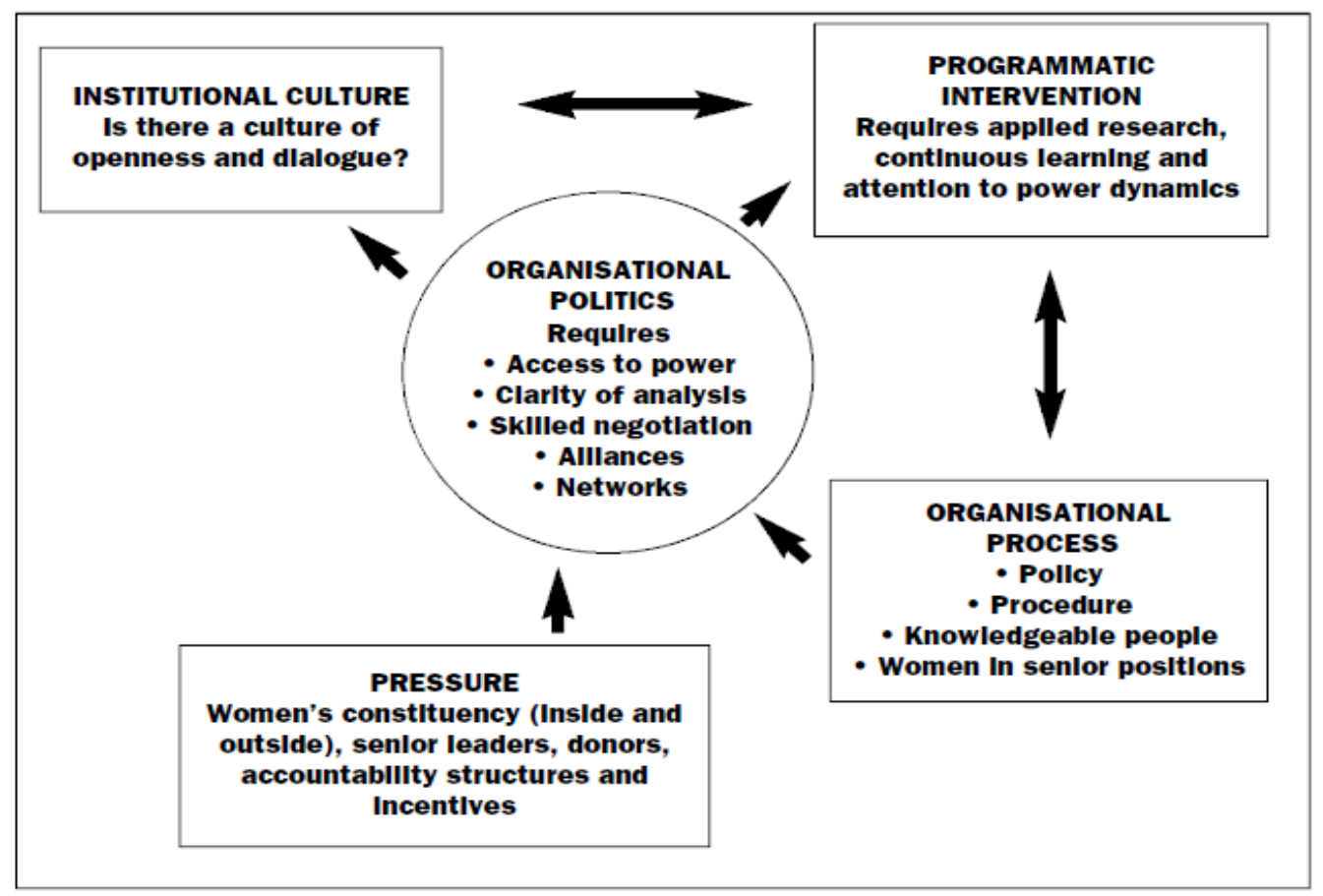

Figure 7.2. The organizational likelihood of promoting gender equality (Rao \& Kelleher, 2005, p. 67)

From the limited scope of this research, it is too early to make a generalization here about the positive impact of the NGOs' gender work on the attitudes, perceptions, and behaviours towards gender. Of the three male staff in total (two in the INGO and one in the LNGO) there were many other factors in the formulation of men's personalities and identities. However, all respondents came to agree that the personal changes in terms of attitude and behaviour towards gender relationships stemmed from their workplace. In other words, there was potential for an NGO to build an enabling environment for this valuable change in a wider 
context. Respondents from both organizations were aware of people's lives outside the work situation, and thus took account of women's reproductive roles while recognizing women and men as parents, partners, family members, and development practitioners. They had both private and public lives. What happened in their private lives impacted on their performance at work. The organizations provided them with flexible working hours and leave arrangements, so that men and women could carry out either reproductive or caring duties in the home. It developed an organizational culture which was sympathetic to people's lives outside the office, allowing people to have feelings and devoting organizational space to discussion of personal matters (Macdonald et al., 1997).

There was a direct correlation between organizational development and the extent to which an organization is able to have an impact on the wider world in which it operates (Macdonald et al., 1997). The domestic sphere was understood to disqualify women from full participation in waged work because their domestic family lives have been overwhelmingly taken care of by women. According to the findings, in many cases, female staff in both organizations refused opportunities for job promotion, further study, and career development because of family burdens. This mattered less for male staff because women's responsibility for the home and children was a subsidy which enabled men to spend long hours at work, to travel at short notice, and to carry on their working lives (Macdonald et al., 1997).

In the other cases, cultures were generally changed by the influence of leaders, and by the understanding of others about the value of the new directions (Rao \& Kelleher, 2005). Strong and educated leaders in the LNGO were perhaps catalysts for this tendency because they were Vietnamese women, wives, and mothers. Thus, they may not only capture a good snapshot of gender structures and norms but also be in position to feel Vietnamese culture in their hearts. As leaders, they found the daily coaching and gender internalizing techniques were the most appropriate for their organization and certainly sprung from their own experiences. The LNGO paid attention to informal cultural arrangements and norms, which were created mainly to engage employees' partners in realistic activities. Therefore, they could have more understanding of gender and their own reflection on gender concerns in a wider society. All these initiatives were mentioned in the INGO but had not yet occurred, 
perhaps for reasons including, inter alia, the over-emphasis on technical dimensions, the size of organizations in terms of staff and geographical coverage, and complication in staffing structures.

Another point to be discussed here is the culture of openness and dialogue in the organizations. The research revealed only limited and superficial communication within and between the organizations about the implementation of gender mainstreaming. This finding was found in the INGO as one of the challenges facing the process. In the organization, some people were active while others did not listen to and accept others' ideas for improvement. Questioning could be interpreted as a way of accusing, challenging, and looking down on someone. Therefore, people tended to be passive in these sharing sessions. The concept of 'losing face' might be another barrier preventing people from sharing more deeply with each other. With partnerships, the idea of getting all partners together to reach proper understanding of notions of gender equality did not occur. Consequently, there was a risk that the misunderstanding or lack of understanding could lead to adopt concepts and opinions uncritically and superficially. This issue could also mean that there would be not enough discussion, argument, and understanding of new ideas(Roche, 2001). INGO 1.1, as GFP staff, expressed his interest in my research as he hoped to see gender mainstreaming in other organizations and to learn from them given, currently, the sharing between organizations with a gender-focus in the development sector in Vietnam was limited.

\subsection{Thoughts for future research}

As noted, most research into gender mainstreaming has been carried out through case studies. This research adds an additional piece to the whole picture of gender mainstreaming in Vietnam in general. Although the UN in Vietnam conducted a gender audit within its system as mentioned in Chapter 3, this research provides an early exploration into the current implementation of gender mainstreaming in the NGO community, both local and international. Given the increasing number of NGOs in Vietnam, it would beneficial to scale up this research to achieve generalizations. Considering the positive impacts of NGOs' working environments on transforming individuals and wider society about gender, more research may investigate how NGOs are dealing with gender and organizational cultures. It also can be a possibility to look into the gender consideration in 
organizational culture in other sectors in Vietnam. Crucial too is more research on how the INGO and the LNGO can cooperate to make the best of their approaches to gender mainstreaming. A good partnership could overcome the conflicts of interest within each organization and match them well.

\subsection{Concluding remarks}

This research represents a critical insight into the implementation of gender mainstreaming in an INGO and a LNGO four years after they officially brought gender to the centre of their work. It highlights some of the technical and institutional challenges and opportunities they have been encountering. I hope this research will provide an example for other organizations in Vietnam, and that they can be provided deeper insights into their current situations. In addition, this research hopes to contribute to the improvement of sharing in the network of NGOs in Vietnam given the importance of communication for the common goal of improving gender inequality in Vietnam. I also hope the research will be able to ring the alarm for the necessity of all organizations to adapt globally accepted strategies and tools within the specific context of Vietnam. In other words, whatever tools and approaches are adopted need to be specifically tailored to local cultured and norms as well as organizational contexts. In so doing, effectiveness could be strengthened and unexpected consequences could be avoidable. 


\section{References}

ADB. (2002). Country Briefing Paper: Women in Vietnam. In S. Fahey, S. Lateef, A. Pio \& N. N. Tuyen (Eds.). Manila, Philippines: Regional and Sustainable Development Department and Mekong Department, Asian Development Bank.

Ali, S. S. (2008). Women's rights, CEDAW and international human rights debates: toward empowerment? In J. D. Momsen (Ed.), Gender and Development (pp. 5-23). The UK: Routledge.

Anh, L. (2011). Vietnam: Unbalanced gender ratio. Retrieved from http://tuoitre.vn/Chinh-tri-Xahoi/441636/VN-mat-can-bang-gioi-tinh-tram-trong.html

AusAID. (1995). Non-Government Organizations: Review of the Effectiveness of NGO Programs. Canberra: AusAID.

Babacan, H. (2004). 'Women hold up half the sky': Gender mainstreaming and women's inequality in Australia. Development Bulletin (64), 49-53.

Bannon, I., \& C.Correia, M. (2006). Gender and Its Discontents: Moving to Men-streaming Development. In I. Bannon \& M. C.Correia (Eds.), Men's Issues in Development, the other half of gender (pp. 245-260). Washington, D.C: The World Bank.

Bebbington, A., Hickey, S., \& Mitlin, D. (2008). Can NGOs make a difference? The challenge of development alternative. London, the UK: Palgrave Macmillan.

Bernard, H. R., \& W.Ryan, G. (2010). Analyzing Qualitative Data: Systematic Approaches. California, the US: SAGE Publications, Inc.

Braidotti, R., Charkiewicz, E., Hausler, S., \& Wieringa, S. (1994). Women, the Environment and Sustainable Development: Towards a Theoretical Synthesis. London, the UK: Zed Books Ltd.

Brinkerhoff, J. M., Smith, S. C., \& Teegen, H. (2007). Beyond the "Non": The strategic space for NGOs in development. New York: Palgrave Macnillan.

Bryman, A. (2008). Social Research Methods (3 ed.). Oxford: Oxford University Press.

Chege, S. (1999). Donors shift more aid to NGOs. Africa Recovery, United Nations, 13\#1(2-3), 6.

Clarke, G. (1998). Non-Governmental Organizations (NGOs) and Politics in the Developing World. Political Studies, 46(1), 36-52.

Cleaver, F. (2002). Men and Masculinities: New Directions in Gender and Development. In F. Cleaver (Ed.), Masculinities Matter! Men, Gender and Development (pp. 1-27). London, the UK: Zed Books Ltd.

Corner, L. (2004). Women transforming the mainstream - A think piece. Development Bulletin (64), 11-16. 
D.Long, L., Ngoc Hung, L., Truitt, A., Phuong Mai, L., \& Nguyen Anh, D. (2000). Changing Gender Relations in Vietnam's Post Doi Moi Era. Vietnam: the World Bank.

Dawson, E. (2005). Strategic gender mainstreaming in Oxfam GB. In F. Porter \& C. Sweetman (Eds.), Mainstreaming Gender in Development: A Critical Review (pp. 80-89). Oxford, UK: Oxfam GB.

Dema, S. (2008). Gender and Organization: The (re)production of gender inequalities within Development NGOs. Women's Studies International Forum, 31(6), 441-448.

Denscombe, M. (2007). The Good Research Guide for small-scale social research project. New York, the US: Open University Press.

Denscombe, M. (2010). The Good Research Guide for small-scale social research projects (Vol. 4). New York, the US: Open University Press.

Denzin, N. K., \& Lincoln, Y. S. (2005). Introduction: the discipline and practice of qualitative research. In N. K. Denzin \& Y. S. Lincoln (Eds.), The Sage Handbook of Qualitative Research (pp. 1-33). California: Sage Publications, Inc.

Desai, V. (2005). NGOs, gender mainstreaming, and urban poor communities in Mumbai. In F. Porter \& C. Sweetman (Eds.), Mainstreaming Gender in Development: A Critical Review (pp. 90-98). Oxford, the UK: Oxfam GB.

Doyle, N. (2002). Why Do Dogs Lick their Balls? Gender, Desire and Change - a Case Study from Vietnam. In F. Cleaver (Ed.), Masculinities Matter! Men, Gender and Development (pp. 186208). London, the UK: Zed Books Ltd.

Eade, D. (2000). Preface. In D. Eade (Ed.), Development, NGOs, and Civil Society (pp. 9-15). The UK: Oxfam GB.

Flood, M. (2004). Men, gender and development. Development Bulletin (64), 26-31.

Fowler, A. (1993). Non-governmental organizations as agents of democratization: an African perspective. Journal of International Development, 5(3), 325-339.

Gibbon, P., \& Bangura, Y. (1992). An introduction to some conceptual and empirical issues. In P. Gibbon, Y. Bangura \& A. Ofstad (Eds.), Authoritarianism, democracy and adjustment: the politics of economic reform in Africa (pp. 7-39). Sweden: Nordiska Afrikainstitutet.

Government. (2011). Decision on approving the National Strategy for Gender Equality 2011-2020. Hanoi, Vietnam: Prime Minister's Office. Retrieved from http://vietlaw.vn/Van-ban/Linh-vuckhac/Quyet-\%C4\%91inh-2351-Q\%C4\%90-TTg-Phe-duyet-Chien-luoc-quoc--.aspx.

Grbich, C. (2007). Qualitative data analysis: An introduction. London, the UK: SAGE.

Harding, S. (1987). Introduction: Is there a feminist method? In S. Harding (Ed.), Feminism and Methodology (pp. 1-14). USA: Indiana University Press. Retrieved from http://books.google.co.nz/books?hl=en\&lr=\&id=w2gzw6zz4flC\&oi=fnd\&pg=PP13\&dq=Hardin 
$\mathrm{g}+(1987) \& o t s=Y D P X y Y o G D B \& s i g=s d a 3 p 7 Y Z$ 18leEqLrHIPew0n5Xg\#v=onepage\&q=Harding\%2 $\underline{0(1987) \& f=\text { false. }}$.

Hesse-Biber, S. N. (2010). Mixed methods research: Merging theory with practice. New York, the US: The Guilford Press.

Hoang, L. A. (2005). Gender relations, intra-household power hierarchies and social norms in migration decision-making in rural Vietnam. University of East Anglia, UK.

Jahan, R. (1995). The Elusive Agenda: Mainstreaming women in development. New Jersey, USA: Zed Books.

Johnson, B., \& Christensen, L. (2008). Educational Research: Quantitative, Qualitative, and Mixed Approaches. London, the UK: SAGE Publications.

Kabeer, N. (1994). Triple roles, gender roles, social relations: The political subtext of gender training frameworks Reversed realities: Gender Hierarchies in Development Thought (pp. 264-305). London: Verso.

Kabeer, N. (1999). From Feminist Insights to an Analytical Framework: An Institutional Perspective on Gender Inequality Institutions, Relations and Outcomes: A Framework and Case Studies For Gender-Awareness Planning (pp. 3-47). London, the UK: Zed Books.

Kabeer, N. (2005). Gender equality and women's empowerment: a critical analysis of the third Millennium Development Goal. Gender and Development, 13(1), 13-24.

Kamat, S. (2004). The privatization of public interest: theorizing NGO discourse in a neoliberal era. Review of International Political Economy, 11(1), 155-176.

Knodel, J., Manh Loi, V., Jayakodil, R., \& Tuan Huy, V. (2004). Gender Roles in the Family: Change and Instability in Vietnam. The US: Population Studies Center, The Institute for Social Research, University of Michigan.

Lyons, T., Curnow, J., \& Mather, G. (2004). Development gender mainstreaming and 'gender respect'. Development Bulletin (64), 37-42.

Macdonald, M., Sprenger, E., \& Dubel, I. (1997). Gender and organizational change: Bridging the gap between policy and practice. Amsterdam: Royal Tropical Institute.

Manderson, L., \& Mark, T. (1997). Empowering women: Participatory approaches in women's health and development projects. Health Care for Women International, 18(1), 17-30.

McGee, R. (2002). Participating in Development, Development Theory and Practice: critical perspective. London, the UK: Palgrave.

MDGIF. (2010). Final Report: Findings and Recommendations. Vietnam: United Nations, Vietnam.

Mercer, C. (2002). NGOs, civil society and democratization: a critical review of the literature. Progress in Development Studies, 2(1), 5-22. 
Mercer, C. (2003). Performing partnership: Civil society and the illusions of good governance in Tanzania. Political Geography, 22(7), 741-763.

Mikkelsen, B. (2005). Methods for Development Work and Research: A New Guide for Practitioner Retrieved from http://books.google.co.nz/books?hl=en\&lr=\&id=SIVdugYuwOIC\&oi=fnd\&pg=PA1990\&dq=Mi kkelsen+2005\&ots=rZfE5EIDje\&sig=T3R3nrEr63D2AZ5C mxK7NAo8IX8\#v=onepage\&q=Mikkel sen\%202005\&f=false

Miller, R. (2009). The Gender and Development Resource Kit. Wellington, New Zealand: Council for International Development.

Mitchell, S. (2000). Women in leadership in Vietnam. Development Bulletin (51), 30-33.

Mitchell, S. (2004). What lies at the heart of the failure of gender mainstreaming: The strategy or the implementation? Development Bulletin (64), 8-11.

MOFA. (2007). International Non-governmental Organizations, from http://www.mofahcm.gov.vn/vi/mofa/ctc quocte/un/nr040819155753/nr060928111253/ns 070731092928/view\#nU3THT|4IR5I

Mohan, G. (2002). The disappointments of civil society: the politics of NGO intervention in northern Ghana. Political Geography, 21(1), 125-154.

Moser, A. (2007). Gender and Indicators: Overview Report. The UK: Institute of Development Studies.

Moser, A., \& Moser, C. (2005). Gender mainstreaming since Beijing: a review of success and limitations in international institutions. In F. Porter \& C. Sweetman (Eds.), Mainstreaming Gender in Development (pp. 11-22). Oxford, the UK: Oxfam GB.

Moser, C. (1993). Gender Planning and Development: Theory, Practice and Training. New York, the US: Rouledge.

Moser, C. (2005). Has gender mainstreaming failed? International Feminist Journal of Politics, 7(4), 576-590.

Moser, C., Tornqvist, A., \& Bronkhost, B. V. (1999). Mainstreaming gender and development in the World Bank: progress and recommendations. Washington, D.C.: World Bank Publications.

MPI. (2008). Vietnam continues to achieve the Millenium Developmeng Goals. Hanoi, Vietnam: The Ministry of Planning and Investment.

Mukhopadhyay, M. (2004). Mainstreaming Gender or "Streaming" Gender Away: Feminists Marooned in the Development Business. IDS Bulletin, 35(4), 95-103.

NA. (2007). Law on Domestic Violence. Hanoi, Vietnam: Vietnamese Government. Retrieved from http://civillawnetwork.wordpress.com/category/vietnam-legal-documents/page/2/. 
NCFAW. (2004a). Gender Mainstreaming Guidelines in National Policy Formulation and

Implementation. Hanoi: Retrieved from http://www.ubphununcfaw.gov.vn/?lang=E\&func=newsdt\&Cat|D=130\&newsid $=1131 \& M N=130$.

NCFAW. (2004b). Gender Mainstreaming Guidelines in National Policy Formulation and Implementation: Toward Gender Equality in Vietnam through Gender-Responsive National Policy and Planning. Hanoi, Vietnam: National Committee For the Advancement of Women.

Ndegwa, S. (1996). The two faces of civil society: NGOs and politics in Africa. The US: West Hartford, Kumarian Press.

Neuman, W. L. (2006). Social research methods: Qualitative and quantitative approaches. Boston: Pearson.

O'Neil, P. (2004). Rethinking gender mainstreaming (or, Did we ditch women when we ditched WID?) - A personal view. Development Bulletin (64), 45-49.

Osirim, J. M. (2001). Making good on commitments to grassroots women: NGOs and Empowerment for Women in Comtemporary Zimbabwe. Women's Studies International Forum, 24(2), 167180.

Ostergaard, L. (1992). Gender, Gender and Development: A practical guide. New York, the US: Routledge.

Pearce, J. (2000). Development, NGOs, and civil society: the debate and its future. In D. Eade (Ed.), Development, NGOs, and Civil Society (pp. 15-44). The UK: Oxfam GB.

Plowman, P. (2003). Organizational change from two perspectives: gender and organizational development. In D. Eade (Ed.), Development Methods and Approaches Critical Reflection (pp. 104-123). Oxford, the UK: Oxfam GB.

Porter, F., \& Sweetman, C. (2005). Editorial Mainstreaming Gender in Development: Critical Review (pp. 2-10). Oxford, the UK: Oxfam GB.

Rao, A., \& Kelleher, D. (2005). Is there life after gender mainstreaming? Gender and Development, 13(2), 57-69.

Rathgeber, M. E. (1990). WID, WAD, GAD: Trends in Research and Practice. The Journal of Developing Areas, 24(4), 489-502.

Razavi, S., \& Miller, C. (1995). From WID to GAD: Conceptual Shifts in the Women and Development Discourse. Geneva, Switzerland: United Nations Research Institute for Social Development, United Nations Development Programme.

Rea, J. (2004). Gender mainstreaming: Getting it right in the workplace first. Development Bulletin (64), 42-45. 
Reid, E. (2004). Transformational development and the wellbeing of women. Development Bulletin (64), 16-21.

Roche, C. (2001). Middle-aged man seeks gender team. In C. Sweetman (Ed.), Beyond Rhetoric: Men's Involvement in Gender and Development Policy and Practice. London, the UK: Oxfam GB.

Rowland, J. (2003). Development methods and approaches: critical reflection. London, the UK: Oxfam GB.

Silverman, D. (2011). Qualitative Research: Issues of Theory, Method and Practice (3 ed.). London, the UK: SAGE.

Stake, R. E. (2005). Qualitative Case Studies. In N. K. Denzin \& Y. S. Lincoln (Eds.), The SAGE Handbook of Qualitative Research (pp. 443-466). London, the UK: SAGE Publications.

Standing, H. (2004). Gender, Myth and Fable: The Perils of Mainstreaming in Sector Bureaucracies. IDS Bulletin, 35(4), 82-88.

Subrahmanian, R. (2009). Making Sense of Gender in Shifting Institutional Contexts: Some Reflections on Gender Mainstreaming. IDS Bulletin, 35(4), 89-94.

Tadele, F. (2001). Men in the kitchen, women in the office? Working on gender issues in Ethiopia. In C. Sweetman (Ed.), Beyond Rhetoric: Men's Involvement in Gender and Development Policy and Practice (pp. 16-20). London, the UK: Oxfam GB.

Teerawichitchainan, B., Knodel, J., Manh Loi, V., \& Tuan Huy, V. (2008). Gender Division of Household Labor in Vietnam: Cohort Trends and Regional Variations. The US: Population Studies Center, The Institute for Social Research, University of Michigan.

Thomas, P. (2004). Introduction: Gender and Development: Bridging policy and practice Development Bulletin (64), 4-8.

Tiessen, R. (2004). Re-inventing the gendered organization: staff attitudes towards women and gender mainstreaming in NGOs in Malawi. Gender, Work \& Organization, 11(6), 689 - 708

Tiessen, R. (2007). Everywhere/nowhere: gender mainstreaming in development agencies. The US: Kumarian Press, Inc.

Tolich, M., \& Davidson, C. (1999). Starting fieldwork: An introduction to qualitative research in New Zealand. Auckland, New Zealand: Oxford University Press New Zealand.

UN. (2009). Gender Mainstreaming Strategy 2009-2011. Vietnam: United Nations Viet Nam.

UN. (2010). Power, voice and rights - key to achieving gender equality in Asia-Pacific. Retrieved from http://www.un.org.vn/en/media-releases/107-un-press-releases/1167-power-voice-andrights-keys-to-achieving-gender-equality-in-asia-pacific.html

UNIFEM. (2006). Promoting Gender Equality in New Aid Modalities and Partnerships. Retrieved from http://www.governo.it/Governolnforma/Dossier/Farmaci bioetica/Rapporto 2006.pdf 
Vijeyarasa, R. (2010). Scrutinizing Vietnam's Progress Towards Gender Equality. Development, 53(1), 91-97.

VNGOs. (2006). Report of Non-Governmental Organization regarding implementation of all forms of discrimination against women (CEDAW) in Vietnam. Hanoi, Vietnam: A group of Vietnamese Non-governmental organizations.

VWU. (2005). Basic Information on Vietnam Women's Union. Retrieved from http://hoilhpn.org.vn/newsdetail.asp?Catld=66\&Newsld=819\&lang=EN

Wallace, T. (1998). Institutionalizing gender in UK NGOs. Development in Practice, 8(2), 159-172.

Wallace, T., \& Wendoh, S. (2005). Rethinking gender mainstreaming in African NGOs and communities. In F. Porter \& C. Sweetman (Eds.), Mainstreaming Gender in Development: $A$ Critical Review (pp. 70-80). Oxford, the UK: Oxfam GB.

WB. (2006). Vietnam Country Gender Assessment. Vietnam: The World Bank Group.

Wells, J., \& McEwan, T. (2004). Gender mainstreaming: Moving from principles to implementation The difficulties. Development Bulletin (64), 31-33.

Werker, E. D., \& Ahmed, Z. F. (2007). What Do Non-Governmental Organizations Do? Retrieved from http://www.hbs.edu/research/pdf/08-041.pdf

Woodford-Berger, P. (2004). Gender Mainstreaming: What is it (About) and Should we Continue Doing it? IDS Bulletin, 35(4), 65-72. 


\section{Appendix 1: Information Sheet}

\section{VICTORIA UNIVERSITY OF WELLINGTON \\ Te Whare Wānanga o te Ūpoko o te Ika a Māui}

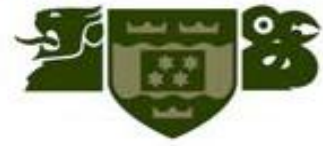

\section{INFORMATION SHEET}

Participant Information Sheet for a Study of Gender Mainstreaming in Development Non-

\section{Governmental Organizations based in Vietnam}

Researcher: Binh Le

School: School of Geography, Environment and Earth Sciences, Faculty of Science, Victoria University of Wellington, New Zealand

I am a Masters student in Development Studies at Victoria University of Wellington. As part of this degree I am undertaking a research project leading to a thesis. The project I am undertaking is examining the extent to which Development Non-governmental organizations (NGOs) in Vietnam mainstream gender in their work within their organization and in the field. The project has been approved by the Victoria University Ethics Committee. I am inviting staff at different levels of four development NGOs to participate in this study. Participants will be interviewed using semi-structured questions. Findings from those interviews will be as a main source of data collection for the research alongside observation and document analysis. The interview can take place when and where you feel comfortable. I will tape-record the interview and you may request that it be turned off at any point in the interview without having to give reasons. You will also have an opportunity to review the transcript of your interview before it is used for analysis purposes.

Responses collected will form the basis of my research project, an academic article and thesis report on a confidential basis. It will not be possible for you to be identified personally and efforts will be made to hide the identity of your organization. All material collected will be kept confidential. No other person besides me and my supervisor, Senior Lecturer - Sara Kindon, will see the research material. The thesis will be submitted for marking to the School of Geography, Environment and Earth Sciences and deposited in the University Library. It is 
intended that one or more articles will be submitted for publication in scholarly journals. Data will be destroyed two years after the end of the project.

Should any participants feel the need to withdraw from the project, they may do so without question at any time before the data analysis is complete. Just let me know within one week after the interviews.

If you have any questions or would like to receive further information about the project, please contact me at lebinh@myvuw.ac.nz or PREFERABLY at lebinh273@gmail.comor my supervisor, Sara Kindon, at the School of Geography, Environment and Earth Sciences at Victoria University, P O Box 600, Wellington, phone +64 4 4636194, email:sara.kindon@vuw.ac.nz

Binh Le

Signed 


\section{Appendix 2: Consent Form}

\section{VICTORIA UNIVERSITY OF WELLINGTON \\ Te Whare Wānanga o te Ūpoko o te Ika a Māui

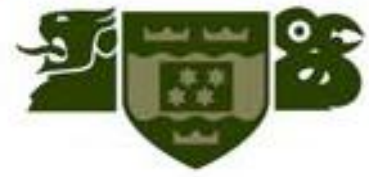

CONSENT TO PARTICIPATION IN RESEARCH

Title of project: Critical Reflections on Gender Mainstreaming in Development Nongovernmental organizations in Vietnam

I have been given and have understood an explanation of this research project. I have had an opportunity to ask questions and have them answered to my satisfaction.

I understand that I may withdraw myself (or any information I have provided) from this project within one week before and after the interview.

I understand that any information I provide will be kept confidential to the researcher, the supervisor and the person who transcribes the tape recordings of our interview. The published results will not use my name. I consent to information or opinions which I have given not being attributed to me in any way that will identify me or my organization.

I may request to have the tape turned off at any point in the interview and will have an opportunity to check the transcript of my interview before it is used in research.

I understand that the tape recording of interviews will be electronically wiped two years after completion of the project unless I indicate that I would like them returned to me.

Signed:

Name:

Address 


\section{Appendix 3: Ethics Approval}

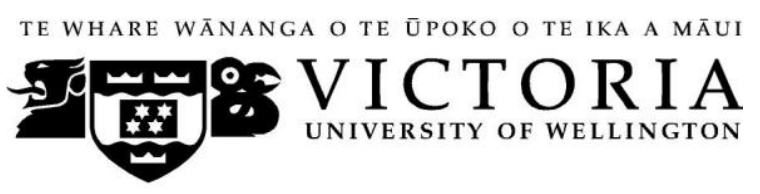

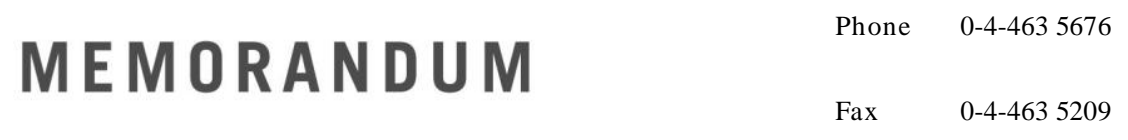

\begin{tabular}{l|l}
\hline TO & Binh Le \\
\hline COPY TO & Sara Kindon \\
\hline FROM & Dr Allison Kirkman, Convener, Human Ethics Committee \\
\hline
\end{tabular}

\begin{tabular}{l|l}
\hline DATE & 12 April 2011 \\
\hline PAGES & 1 \\
\hline
\end{tabular}

\begin{tabular}{l|l}
\hline SUBJECT & $\begin{array}{l}\text { Ethics Approval: } 18392 \text { Critical reflections on gender } \\
\text { mainstreaming in development non-governmental } \\
\text { organizations (NGOs) in Vietnam }\end{array}$ \\
\hline
\end{tabular}

Thank you for your application for ethical approval, which has now been considered by the Standing Committee of the Human Ethics Committee.

Your application has been approved from the above date and this approval continues until 28 February 2012. If your data collection is not completed by this date you should apply to the Human Ethics Committee for an extension to this approval.

Best wishes with the research.

Allison Kirkman
Human Ethics Committee 